\title{
Carbon-based SERS biosensor: from substrate design to sensing and bioapplication
}

\author{
Xiu Liang ${ }^{1,2}$, Ning Li ${ }^{1}$, Runhao Zhang ${ }^{1}$, Penggang Yin ${ }^{3}$, Chenmeng Zhang ${ }^{3}$, Nan Yang ${ }^{3}$, Kang Liang ${ }^{4}$ and Biao Kong ${ }^{2}$
}

\begin{abstract}
The sensing of bioactive molecules based on photochemical techniques has become one of the fastest-growing scientific fields. Surface-enhanced Raman scattering (SERS) is a highly sensitive technique for the detection of lowconcentration molecules, including DNA, microRNA, proteins, blood, and bacteria; single-cell detection and identification; bioimaging; and disease diagnosis, providing abundant structural information for biological analytes. One rapidly developing field of SERS biosensor design is the use of carbon-based nanomaterials as substrate materials, such as zero-dimensional carbon quantum dots, one-dimensional carbon nanotubes, two-dimensional graphene, and graphene oxide (GO) and three-dimensional spatial carbon nanomaterials or carbon-based core-shell nanostructures. In this review, we describe the recent developments in SERS biosensors, in particular carbon-based SERS, for the detection of bioactive molecules. We systematically survey recent developments in carbon nanomaterial-based SERS biosensors, focusing on fundamental principles for carbon-based materials for SERS biosensor design, fabrication, and operation, and provide insights into their rapidly growing future potential in the fields of biomedical and biological engineering, in situ analysis, quantitative analysis, and flexible photoelectric functional materials. As such, this review can play the role of a roadmap to guide researchers toward concepts that can be used in the design of nextgeneration SERS biosensors while also highlighting current advancements in this field.
\end{abstract}

\section{Introduction}

The development of ultrasensitive biosensors with femto-, pico-, and nano-sensitivity is of great significance not only in medical diagnostics, such as cancer biomarker identification for early diagnosis of disease, biomedical monitoring, and therapy, but also in biomolecular imaging and analytical chemistry sensing. Therefore, great research effort has been focused on designing biosensors with diverse analytical technologies, such as electrochemistry techniques $^{1-4}$, chemiluminescence immunoassay ${ }^{5-9}$, chromatography techniques ${ }^{10-12}$, or a combination of these techniques $^{13-16}$. The gold standard in bioanalysis

\footnotetext{
Correspondence: Biao Kong (bkong@fudan.edu.cn)

${ }^{1}$ National Supercomputer Research Center of Advanced Materials, Advanced Materials Institute, Qilu University of Technology (Shandong Academy of

Sciences), 250014 Jinan, People's Republic of China

2Department of Chemistry, Laboratory of Advanced Materials, Shanghai Key Laboratory of Molecular Catalysis and Innovative Materials, Fudan University, 200433 Shanghai, People's Republic of China

Full list of author information is available at the end of the article
}

technology is associated with the characteristics of a simple sample pretreatment process, high stability during treatment, label-free and rapid detection, and so on, which should be considered when designing efficient and quick technical means suitable for complex biological environment systems. Among the different sensing techniques, Raman spectroscopy enables rapid, precise in situ molecular identification with the extremely low Raman activity of water molecules, yet the limitation is the rather weak Raman signal caused by the small cross-section of commonly detected molecules. Fortunately, surface-enhanced Raman scattering (SERS) ${ }^{17}$ is capable of detecting molecules even at the single-molecule scale on or near the surface of plasma nanostructures, which greatly expands the application scope of standard Raman spectroscopy ${ }^{18-21}$. In addition, the plasma biosensor allows ease-of-use, fast testing (within 5-15 $\mathrm{min}$ ) with superior sensitivity and multiple capabilities ${ }^{22}$. To use SERS to detect single cells stably and reliably in practical applications, it is necessary 


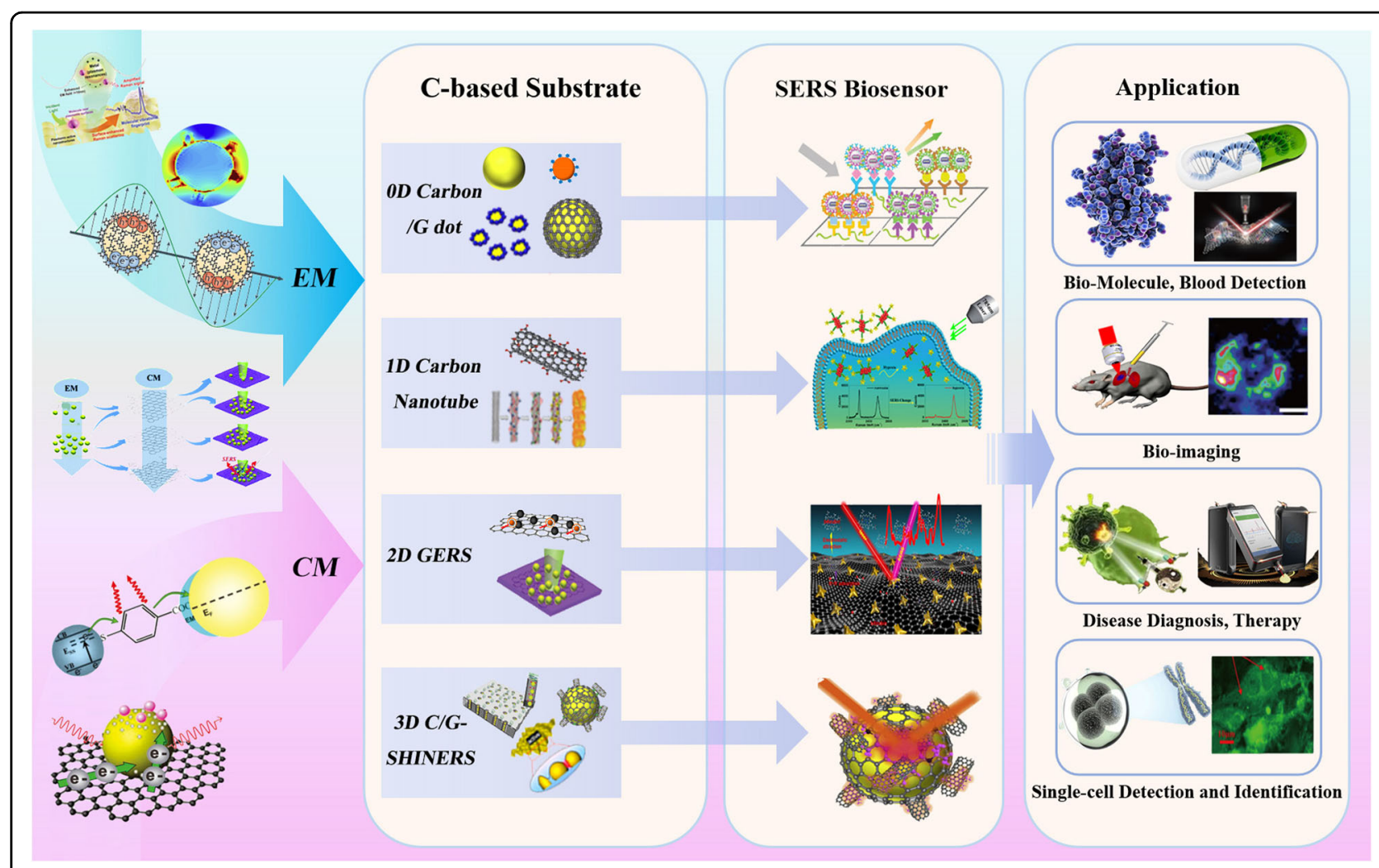

Fig. 1 Schematic illustration of the main content of carbon-based nanomaterials as SERS biosensors with two main enhancement mechanisms in different biological application fields in this review.

to control the stability of nanostructures and plasma effects around hot spots to limit the fluctuation in SERS signals. This requires us to better understand the origin of highly enhanced single-cell signals. As a powerful analysis technique, SERS technology depends largely on the substrate materials, ranging from traditional noble metals to semiconductor nanomaterials or novel nanocomposite materials. SERS-based signal detection and molecular recognition will provide unprecedented opportunities for research in biomedicine, life sciences, analytical chemistry, and other fields ${ }^{23-26}$. It is worth noting that with the isolation of carbon nanotubes (CNTs) in 1991 and graphene in $2004^{27-30}$, the number of articles published in the field of analytical sensors based on carbon-based SERS nanomaterials has increased rapidly ${ }^{31-36}$. However, a definitive discussion that focuses on various carbon nanomaterials in the important field of SERS biosensing has not been presented. Here, we provide a comprehensive overview of the basic principles of the design, manufacture, and operation mechanism of carbon-based materials for SERS biosensors, and we summarize their rapid growth in the fields of biomedicine, bioengineering, and flexible photoelectric functional materials to provide insights into the future potential of carbon-based SERS biosensors (Fig. 1). This review starts with a brief introduction to the carbon nanomaterial structure and properties of and recent progress in each carbon-based SERS nanomaterial, followed by various surface functionalization procedures and design principles considering the availability, sensitivity, and biocompatibility of carbonbased SERS biosensors. Finally, we describe the key challenges and opportunities in future carbon-based SERS biosensor research.

\section{Carbon-based SERS-active materials}

Since the discovery of fullerene by Sir H. Kroto et al. in 1985 as the first member of the carbon nanomaterial family $^{37}$, this class of low-dimensional nanomaterials has aroused great interest in the past 30 years. Carbon nanomaterials ${ }^{38,39}$, which mainly include fullerene, CNTs, graphene, carbon dots (CDs), and nanodiamonds, have received much attention ${ }^{40,41}$. The first three kinds of carbon nanomaterials mainly consist of $\mathrm{sp}^{2}$ carbon atoms as a seamless network of conjugated $\pi$ electrons, while CDs possess $\mathrm{sp}^{2}$ and $\mathrm{sp}^{3}$ carbon atoms mixed with defects and heteroatoms, and nanodiamonds mainly consist of $\mathrm{sp}^{3}$ carbon atoms. In the past decade, carbon nanomaterials have experienced a burst of activity in biological 
medical research as effective biosensors, which have the advantages of small size, unique optical properties, and large surface area ${ }^{42-45}$.

\section{Zero-dimensional (OD) carbon nanomaterials: CDs}

CDs, a new type of fluorescent nanomaterial, which are also known as carbon quantum dots (CQDs), carbon nanodots, or graphene QDs (GQDs), usually refer to carbonaceous, graphitic nanoparticles (NPs) with particle sizes $<10 \mathrm{~nm}^{40,46,47}$. From the perspective of a chemical structure and physical properties, QDs are similar to graphene oxide (GO), and the difference between them mainly comes from the size difference. In addition, it is also observed that the fluorescence of CDs is related to the excitation, and with an increase in the excitation wavelength, the peak emission wavelength of CDs can be adjusted over the whole visible window range of $400-750 \mathrm{~nm}^{48,49}$. Consequently, they have good potential in fluorescence imaging, in inorganic ion detection, and as photon trapping materials because of their high light emission, low toxicity, and stable fluorescence emission $^{50-52}$. Optical coding of fluorescent NPs has shown wide application prospects in molecular biology, cell biology, cell imaging, medical diagnosis, highthroughput drug screening, combinatorial chemical synthesis, and so on ${ }^{53}$. To improve the sensitivity and convenience of analysis, a bifunctional point that combines SERS and fluorescent components for a variety of analyses has been developed ${ }^{54-56}$. Although SERS has excellent multidetection ability, its slow imaging speed is the main obstacle to the fast recognition of specific markers. Consequently, fluorescence-SERS (F-SERS) has been developed as a new kind of dual-modal nanoprobe to address this problem with faster and more intuitive fluorescence imaging speed and better efficiency than SERS imaging. F-SERS is used as a direct indicator in molecular recognition, and SERS signals are then used as characteristics of specific molecules in multiple interaction applications. Tian's group conjugated fluorescent molecules, such as fluorescein isothiocyanate, to labeled organosilicon shells by simple mixing. In another approach, electrostatic forces help layer-by-layer deposition for fluorescent decoration via interaction with the metal nanosubstrates and QDs attached by the reporter ${ }^{57}$. As depicted in Fig. 2a, c, F-SERS tags combined with a spectral encoding method as a new optical encoding approach were demonstrated by using organicmetal-QD hybrid NPs termed OMQ NPs with a nanolayered structure (Fig. 2b), which made it more feasible to implement very large coding in practical applications through two distinct characteristics compared with a single encoding protocol based on fluorescence or SERS (Fig. 2d). This approach employs SERS and fluorescence spectra as encoding elements instead of individual optical signals, thus broadening the spectral range and improving the encoding efficiency. Another method is to assemble the SERS reporter and fluorescent reagent on different layers of OMQ NPs ${ }^{58}$.

Compared to the above tags, especially QD tags, SERS tags have two main advantages. One is that they are highly sensitive to fluorescence, sometimes exceeding 2 or 3 orders of magnitude for biological samples. The second advantage is due to the multiple detection ability of narrowband spectra. In the past few years, a few classes of GQD- or CQD-based nanocomposites as effective SERS biosensors have been fabricated, which are summarized in Table 1. CQDs, as a useful analytical and sensing platform, have attracted wide attention due to their wide color range, high fluorescence brightness, good stability, biocompatibility, low cytotoxicity, inexpensive and easily available reagents, and simple synthesis methods ${ }^{59}$. The combination of CQDs with plasma metal NPs, such as Ag-(PS-PSS)/C-dot nanocuboids (NBs), C-dot-Ag-NPPDMS, and Ag/PATP@SiO ${ }_{2} / \mathrm{CND}$ NPs, is widely used in the fields of photonic and SERS biosensing. Due to the quantum confinement and edge effect in graphene structures $<100 \mathrm{~nm}$ in size, GQDs have unique photoelectric properties different from those of large graphene. Theoretical calculations show that the surface plasmon frequencies of GQDs are similar to those of metal NPs, but graphene sheets do not have these frequencies. In other words, GQDs can be applied as SERS substrates through electromagnetic enhancement $(E M)^{60-62}$. However, GQDs with quantum confinement properties are prone to fluorescence ${ }^{63,64}$. The photoinduced chargetransfer effect has been studied extensively in many scientific fields for its importance in understanding the photophysical and photochemical properties of excited states in organic molecules. It can be produced by rapid delocalization of charges generated on donors and receptors (not limited to molecules) ${ }^{65}$. In the field of SERS, light-induced charge transfer is an important mechanism between molecules and substrates that can enhance Raman signals ${ }^{66-69}$. This is the key factor in making QDs suitable SERS substrates. However, the Fermi level of QDs (in a vacuum) is $\sim 4.2-4.4 \mathrm{eV}$, and the gap is $\sim 0.4 \mathrm{eV}$. This also limits the application of QDs in SERS technology. The methods of adjusting energy levels and energy gaps are mainly focused on heteroatom doping or self-assembly ${ }^{70-72}$. For example, $\mathrm{Mn}_{3} \mathrm{O}_{4}$ is a potentially suitable additive for facilitating the chargetransfer process in SERS systems ${ }^{73-76} \cdot \mathrm{Mn}_{3} \mathrm{O}_{4}$ exhibits absorption in the ultraviolet and visible regions of 220-730 nm due to the combined effect of charge transfer from $\mathrm{O}_{2}$ to $\mathrm{Mn}$ ions and the crystal field $d$ to $d$ transition $^{77}$; consequently, it has aroused the interest of researchers as a SERS sensor (Table 1). GQD- $\mathrm{Mn}_{3} \mathrm{O}_{4}$ nanocomposites were prepared by cutting multiwalled 

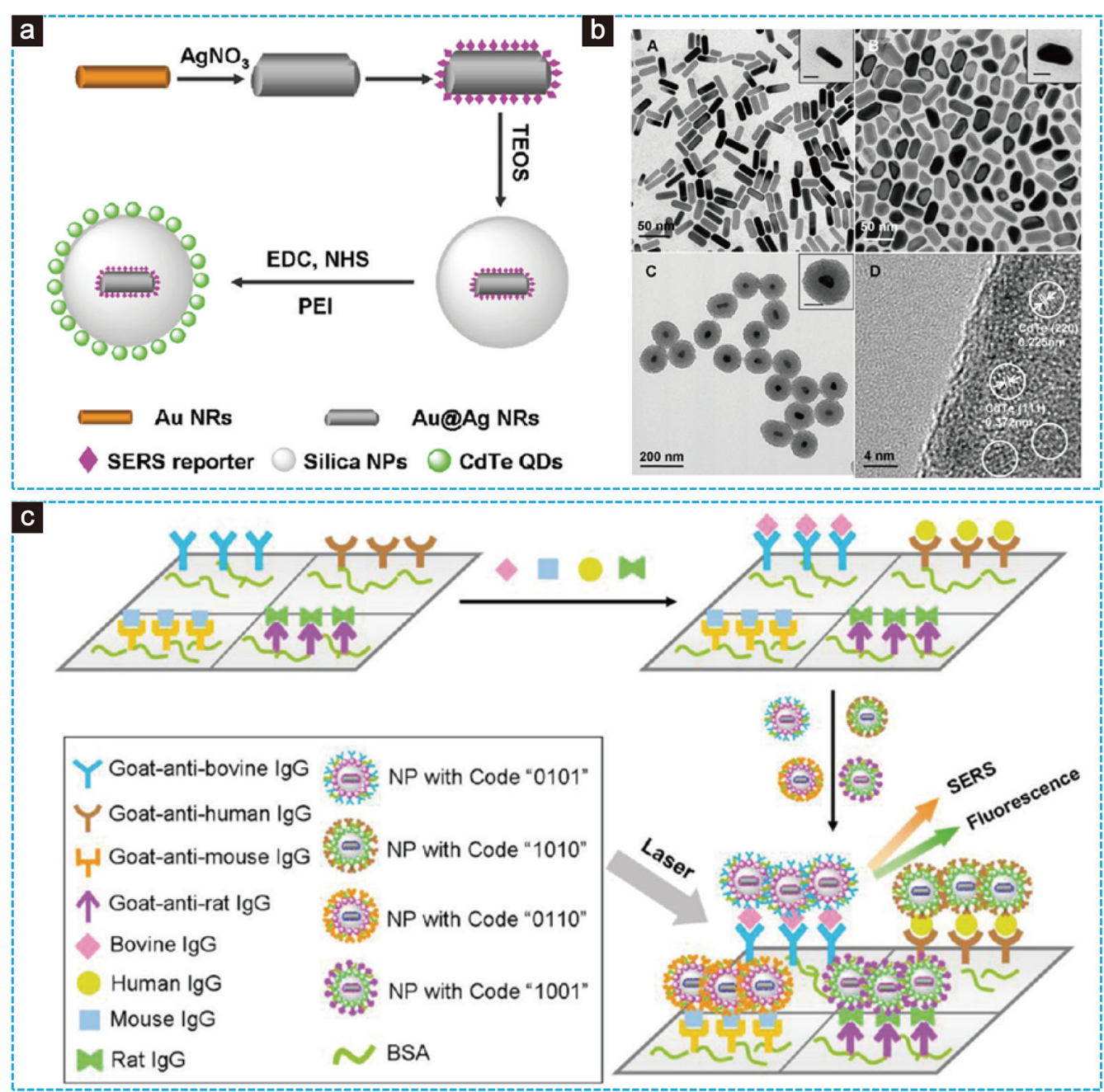

d
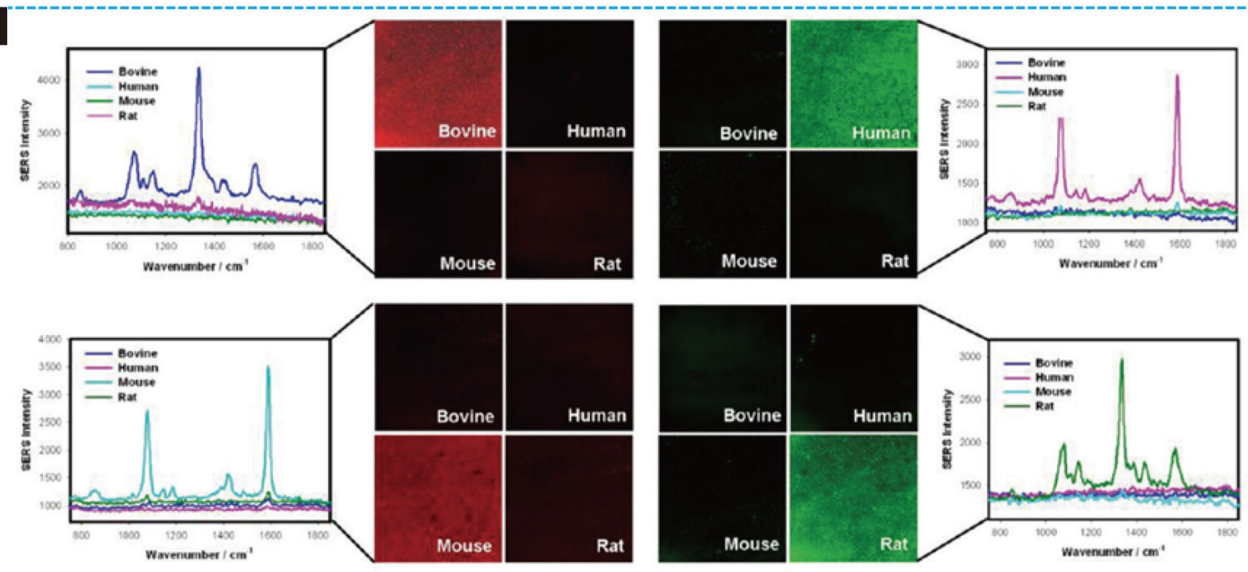

Fig. 2 Typical F-SERS tags with different optical encoding methods. a Schematic illustration of the preparation of organic-metal-QD hybrid nanoparticles (OMQ NPs). b TEM images of Au NRs (A), Au@Ag NRs (B), and OMQ NPs (C), and HRTEM image of CdTe QDs adsorbed on the surface of an OMQ NP (D). The white circle indicates a single CdTe QD. c Schematic illustration of the multianalyte immunoassay based on synthesized encoding carriers using the sandwich structure concept. $\mathbf{d}$ Fluorescence images and SERS results of four sandwich assays, which were pipetted with goat-antibovine lgG-modified 0101 code (top-left), goat-anti-human IgG-modified 1010 code (top-right), goat-anti-mouse IgG-modified 0110 code (bottom-left), and goat-anti-rat lgG-modified 1001 code (bottom-right) ${ }^{57}$. Copyright@ 2012, American Chemical Society. 
Table 1 Recent high-performance reports utilizing QD-based SERS sensors of various biomolecule platforms.

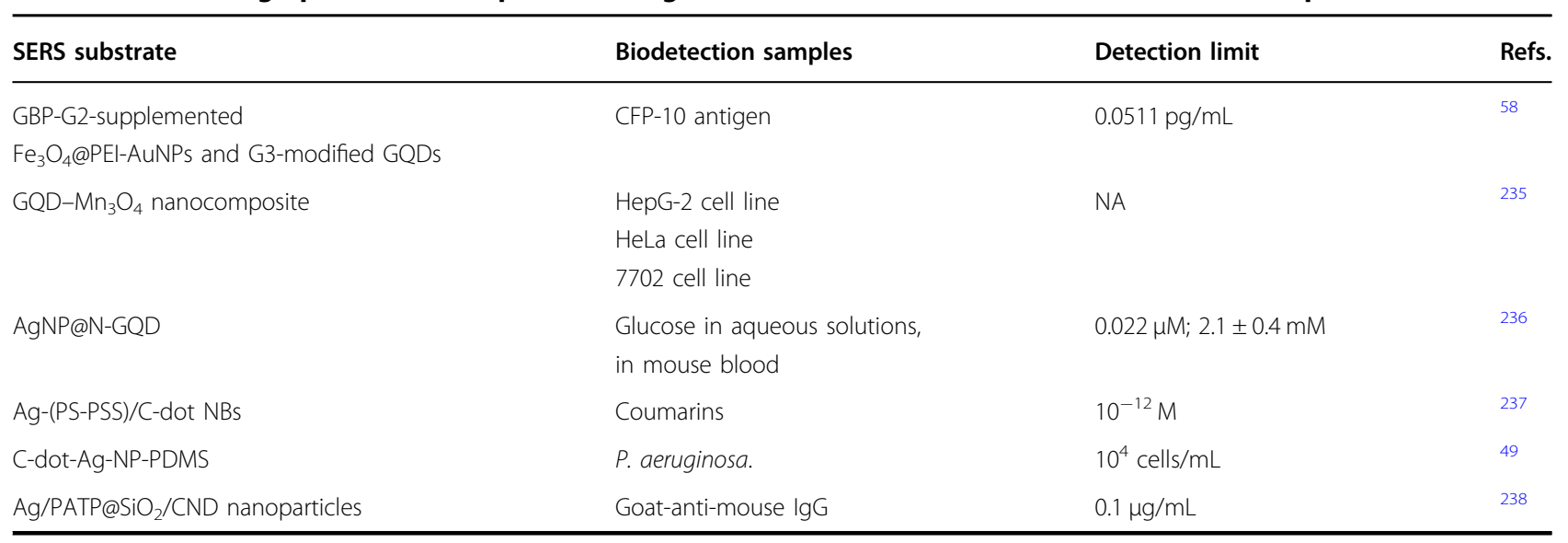

NA not applicable.

CNTs (MWCNTs) into GQDs, further binding them with manganese ions, and employing a $0 \mathrm{D}-3 \mathrm{D}$ self-assembly process. Here, $\mathrm{Mn}_{3} \mathrm{O}_{4}$ is used not only for assembly with GQDs but also for quenching the fluorescence of the GQDs efficiently and suppressing the Raman spectral fluorescence background. Consequently, the prepared GQD- $\mathrm{Mn}_{3} \mathrm{O}_{4}$ nanocomposites have good biocompatibility and can be used to discriminate cancer cells from normal cells (HeLa, HepG-2, and 7702 cell lines) by SERS for potential diagnostic value.

One-dimensional (1D) carbon nanomaterials: CNTs

Since the first discovery of CNTs by S. Iijima in $1991^{78}$, extensive research has been carried out on these 1D carbon materials. CNTs can be thought of as graphene sheets rolled up in different ways, forming dense vertically oriented graphite arrays ("walls") separated by hundreds of nanometer spacers. The calculation of the band structure shows that CNTs are metal compounds, zerogap semiconductors, or semiconductors. Usually, they are divided into metal and semiconductor tubes ${ }^{79-81}$. Therefore, CNTs exhibit different electronic properties, and their incorporation into electronic devices is rather promising. Raman spectroscopic techniques as well as highresolution transmission electron microscopy have been the most important tools for observation of CNTs and can be used to distinguish single-walled nanotubes (SWNTs) from multiwalled nanotubes by different parameters of the average tube diameter or Raman spectra ${ }^{82}$. On the one hand, CNTs have a high surface area of $1000 \mathrm{~m}^{2} / \mathrm{g}$ due to the nanostructures on their surface ${ }^{83}$; on the other hand, the high specific surface area provides many binding sites with gold $(\mathrm{Au})$ or other NPs to obtain a uniform and dense distribution of hot spots over the entire substrate surface $^{84}$. Furthermore, CNTs seem to be biocompatible, which is essential for good SERS sensing of biological objects $^{85}$. All these properties are vital for effective SERS biosensor substrates with a high average enhancement effect. CNTs have no metallic impurities, and direct immobilization of biomolecules (proteins) onto CNTs has been proven unstable. The 1D characteristic of CNTs makes it difficult to controllably assemble and integrate electronic structures into them. Single-walled CNTs (SWCNTs) exhibit strong resonance Raman scattering due to their 1D nature and the electron transition between Van Hove singularities ${ }^{86-88}$. The large Raman scattering cross-section of SWCNTs makes them a promising Raman label and probe for in vitro and in vivo biomedical imaging ${ }^{89-91}$. These features make CNTs one candidate for SERS biosensors but to some extent limit the applications of CNTs. Consequently, the integration of 1D CNTs with noble metal nanomaterials in complex SERS sensors or other devices has achieved great progress through conventional microfabrication approaches. Recently, various CNT materials have been used for SERS measurements by a few research groups. The Tsvetkov group $^{92}$ applied CNT-silver (Ag) NPs as SERS substrates prepared by an electrochemical deposition strategy and obtained an enhancement factor (EF) of 50-2500. The morphology of CNTs coated with solid Ag film promotes strong inhomogeneous localization of the light radiation incident on the substrate, and the formation of a large number of hot spots enhances the Raman signal by up to $5 \times 10^{4}$. The excellent performance of CNT-Ag shows wide potential for highly sensitive SERS molecular diagnosis.

To meet the increasing demands for applications of DNA recognition, biocompatibility systems, and energy conversion/storage devices, efforts have been made to improve the reproducibility and sensitivity of carbonbased materials. For SWCNTs, one important method is to hybridize SWCNTs with other nanostructures (such as graphene, fullerene, and QDs) to achieve multifunctional heterostructures. However, most of the methods are implemented with randomly arranged SWCNTs, which 
conforms to neither the bottom-up principle of molecular electronics nor the expected versatility. To date, using self-assembly or possibly directional assembly to achieve controllable positioning and bonding of SWCNTs is still a huge challenge. Based on the unique carbon chemical characteristics of SWCNTs, a novel method for covalently linking SWCNTs to GNP surfaces was designed by Liu et al. ${ }^{93}$ (Fig. 3a, b). The product, a GNP-CNT heterostructure, has an abundant carbon-based surface area and shows great potential in plasma sensing and imaging applications. In addition, laser-induced plasmon resonance of nanostructures exhibits a remarkable local field enhancement effect, which is widely used to increase the incidence of light in optical processes and realize wide applications ranging from high-sensitivity sensing to highresolution imaging. As depicted in Fig. 3c, d, a Ramanbased temperature measurement method was demonstrated for detection. A quantitative study of thermal properties is the basis of exploring and utilizing local thermal effects ${ }^{94}$. In this study, AuNP arrays that can generate plasma effects were fabricated by dispersing MWCNTs on AuNP arrays. The local temperature and thermal transport properties of CNTs heated by plasmons were studied by surface-enhanced Raman thermometry. This kind of plasma heating effect by suppressing plasmon oscillation is expected to be used in many sensing applications. As shown in Fig. 3e, f, SWCNT metal nanocomposites were synthesized by in situ DNAtemplated Ag NP (AgNP) synthesis and the gold layer growth method for the first time ${ }^{95}$. The design and implementation are a new kind of biofunctionalization method that covalently anchors SWCNTs on the surface of GNPs and forms a unique nanocomposite structure, namely, GNP-CNT, whose surface functional sites increase effectively. The unique biotin-streptavidin-based conjugation chemistry not only endows heterostructure products with high-performance fluorescence properties but also enables them to have surface plasmon properties for SERS chemical sensors.

\section{Two-dimensional (2D) carbon nanomaterials: graphene-enhanced Raman scattering (GERS)}

Recently, graphene and other 2D materials were developed for use as Raman enhancement substrates, which is related to their unique single sheet of carbon atoms in a $2 \mathrm{D}$ honeycomb crystal structure of electrons and phonons with one $2 p_{z}$ orbital of each $\mathrm{sp}^{2}$ hybridized carbon atom constituting a large delocalized $\pi$ bond, forming an ideal flat surface and strong chemical interaction with many organic molecules ${ }^{96,97}$. Consequently, graphene makes it possible to study the chemical enhancement mechanism (CM) independently as a SERS platform. Graphene can enhance Raman signals of adsorbed molecules, and these substrates have been proven to be promising materials for the detection of micro- or trace species. This effect, known as GERS, was first discovered by Ling et al. in $2010^{98}$ (Fig. 4a, b). When graphene is treated with organic solvents, many "emerging bands" appear on mechanically exfoliated graphene, which are distributed among unknown organic compounds contained in the transparent tape used for graphene exfoliation ${ }^{99}$. However, no clear Raman signal was found in the residual region on the $\mathrm{SiO}_{2} / \mathrm{Si}$ substrate. Therefore, it is presumed that graphene has a Raman enhancement effect on trace residues. Considering some important advantages of the graphene matrix, such as uniformity, reproducibility, cleanliness, and low detection limit for aromatherapy dyes, GERS is suitable for both basic studies of SERS effects and many practical areas. The introduction of graphene extends SERS applications of Raman-enhanced substrates in a more controllable and quantitative way.

GERS substrates, as a special kind of SERS substrate, have some unique characteristics compared with traditional SERS substrates. The factors studied in GERS systems can be divided into three categories: molecule, graphene, and excitation laser energy ${ }^{100}$. Factors related to graphene include the graphene thickness or layer number (or first-layer effect) ${ }^{101}$, the graphene Fermi level $^{102}$, the molecular selectivity ${ }^{103}$, and graphene derivatives $^{104}$. In addition, the dependence of the GERS effect on the laser wavelength and polarization is also considered. For molecular selectivity, a schematic illustration of the molecular selectivity in GERS with different types of molecules M1, M2, M3, and M4 is shown in Fig. 4d. According to the two main characteristics of molecules, namely, the molecular energy level and molecular structure, the selection rules are discussed. In particular, EFs involving molecular energy levels require the energies of the highest occupied molecular orbital and the lowest unoccupied molecular orbital to be within an appropriate range relative to the Fermi level of graphene, and this enhancement effect can be explained. The EFs of molecular structure selection show that the molecular symmetry and substituents similar to the graphene structure are beneficial to the enhancement of GERS. The validity of these factors can be explained by group theory and the charge-transfer interaction between molecules and graphene. These results are of great significance for understanding the interaction between graphene and molecules and the chemical mechanism of Raman enhancement. These factors give GERS substrates many advantages, such as high sensitivity, stabilization, and uniformity of molecules on graphene, molecular selectivity, graphene Fermi-level dependence, and fluorescence quenching effects $^{98,103,105}$. For fluorescent dyes adsorbed on graphene (e.g., rhodamine 6G (R6G)), 2D carbon atoms and $\mathrm{sp}^{2}$ aromatic molecules form a system with a $\pi-\pi$ interaction (Fig. 4c). Only one strong fluorescent background 


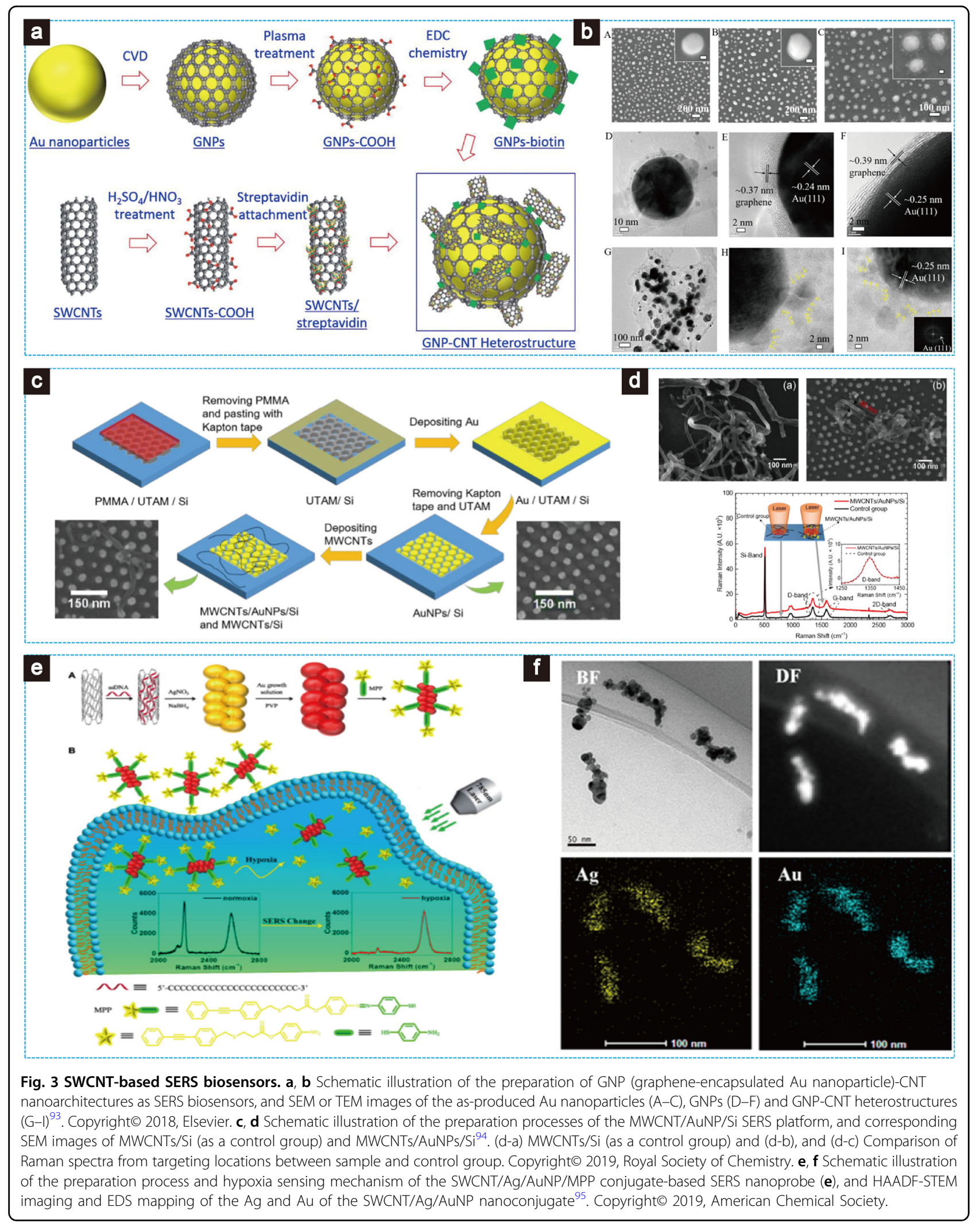




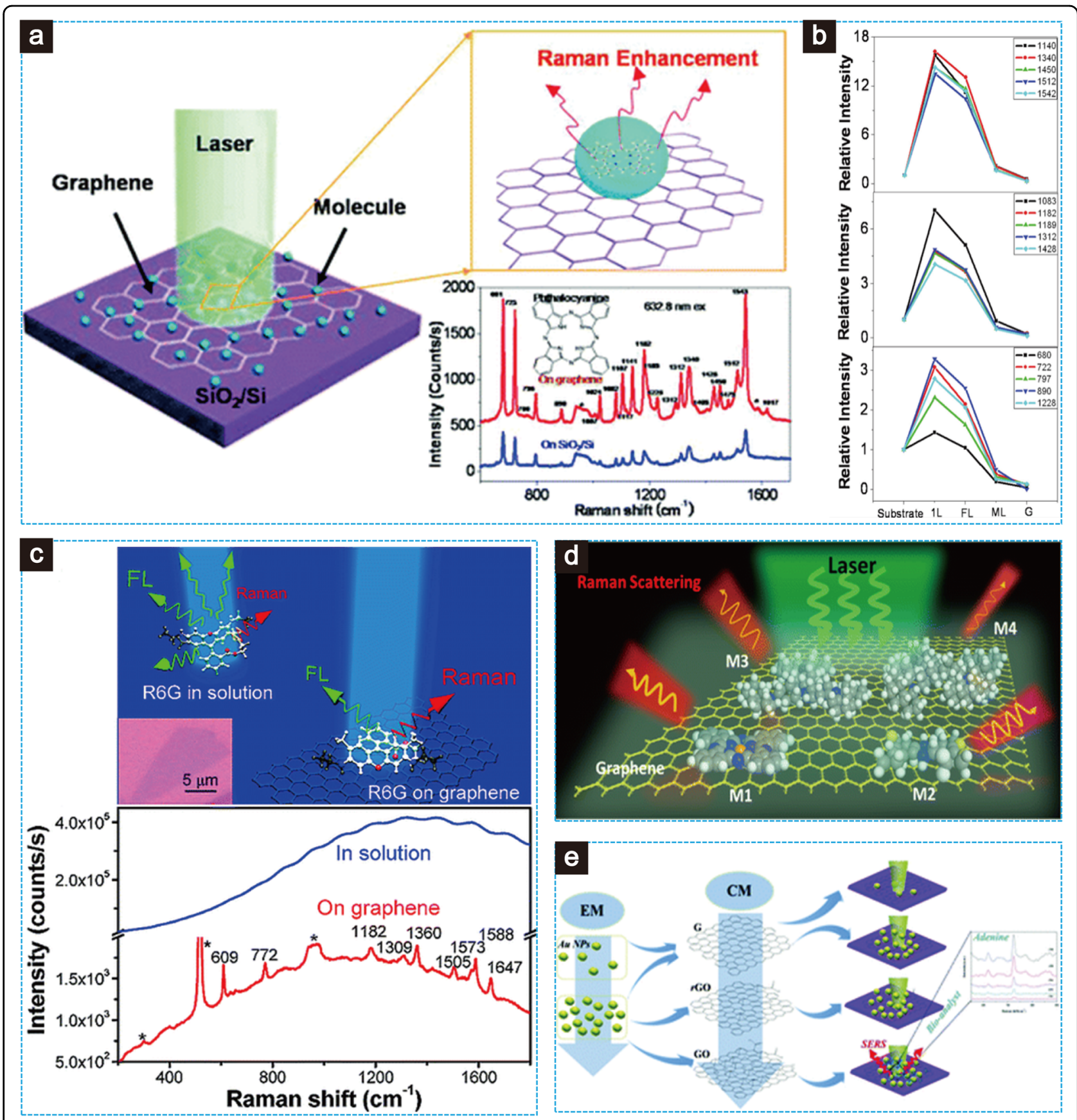

Fig. 4 GERS effects. a, b Schematic illustration of molecules on graphene and a $\mathrm{SiO}_{2} / \mathrm{Si}$ substrate, and Raman experiments ${ }^{98}$. Copyright@ 2010, American Chemical Society. c Schematic illustration of the fluorescence quenching effect of graphene. The inset is an optical image of $1 \mathrm{~L}$ graphene on $\mathrm{SiO}_{2} / \mathrm{Si}$ and the corresponding Raman fluorescence spectra of R6G in water $(10 \mu \mathrm{M}$ ) (blue line) and R6G on $1 \mathrm{~L}$ graphene (red line) under $514 \mathrm{~nm}$ excitation ${ }^{168}$. Copyright@ 2009, Royal Society of Chemistry. d Schematic illustration of the molecular selectivity in GERS. Different types of molecules M1, M2, M3, and M4 are shown on graphene ${ }^{103}$. Copyright $\odot$ 2015, American Chemical Society. e Plasma and chemical enhancement tuning of graphene-based Au hybrids as a SERS biosensor platform ${ }^{107}$. Copyright@ 2016, Royal Society of Chemistry.

has been observed. Because of the strong shot noise emitted by fluorescence, the intensity of the Raman peak should be higher than the detection limit in this system. Successful observation of resonance Raman spectroscopy shows that graphene can be used as a substrate to inhibit fluorescence in resonance Raman spectroscopy of fluorescent molecules and has potential application value in low-concentration detection and resonance Raman spectroscopy of fluorescent molecules. Xie et al. ${ }^{106}$ also revealed that the fluorescence quenching effect for the 
smallest R6G adsorbed on graphene is on the order of $10^{3}$. GERS substrates provide a direct and effective method to measure the Raman scattering spectra of fluorescent dyes under resonance excitation, which are usually difficult to obtain. In terms of application, the ability of graphene is expected to have great application prospects in the process monitoring of chemical and biological systems. Although the validity of graphene as a sole SERS substrate has been confirmed, the limitations of its enhancement effect cannot be ignored. Therefore, in practical applications, graphene-based noble metal composites have attracted great interest from researchers for related materials and mechanisms due to their high sensitivity. Because the different preparation methods of graphene have difficulty tuning the SERS enhancement effect produced by chemical enhancement and plasma enhancement, the effective utilization of SERS is hindered. In our previous work, effective methods of adjusting the EM and $\mathrm{CM}$ effects of graphene-based Au hybrids were developed (Fig. 4e $)^{107}$. First, the oxygen-containing groups on the surface of graphene grown by chemical vapor deposition (CVD) play an important role in the in situ nucleation of $\mathrm{Au}$. The EFs are tunable, which is due to the plasma coupling effect between near AuNPs. Second, GO/Au and reduced $\mathrm{GO}(\mathrm{rGO}) / \mathrm{Au}$ hybrids were grown by in situ crystallization of chemically exfoliated monolayer GO. The SERS performance of CVD-G/Au, rGO/Au, and GO/ $\mathrm{Au}$ shows an increasing trend of EFs, and the observed EFs depend significantly on the degree of chemical bonds on the surface of graphene, indicating that the chemical groups in graphene-based Au hybrids can stably control the chemical enhancement. This indicates that the chemical reinforcement can be controlled by the chemical group stability in graphene-based Au hybrid systems. Most noteworthy is that the optimized GO/Au can detect adenine biomolecules with high sensitivity and a detection limit of $10^{-7} \mathrm{M}$ with good reproducibility and uniformity. This study has important guiding significance for understanding the reinforcement mechanism of composite systems and further expanding the application of graphene-based SERS sensors.

\section{GERS biosensors}

Graphene is the basic component of OD fullerenes, 1D CNTs, and 3D graphite. Graphene has attracted great interest from scientists because of its unique planar structure and novel electronic and optical properties. Graphene as a SERS substrate could be used to investigate the CM independently, and the complex mechanism was deeply explored. In addition, graphene overcomes some limitations of typical SERS substrates and widens their applications in many fields. Especially for bioapplication, graphene with proper modification could exhibit improved biocompatibility and reduced cytotoxicity for diagnosis, elevating the effects in drug delivery, bioimaging, photothermal therapy, and chemotherapy. Due to the limitation of the $\mathrm{CM}$ enhancement capability, graphene-only SERS substrates were mainly employed to study the interaction between graphene and molecules to further understand the CM effect of GERS, and quantitative analysis was also achieved in some cases. Liu et al. ${ }^{108}$ discovered that the $\mathrm{pH}$ influences the SERS intensities of some aromatic molecules on GO, and this is probably due to the different enrichment capacities of GO for these aromatic molecules at different $\mathrm{pH}$ values. In addition, the results also indicated that the GO-mediated SERS is associated with charge-type selectivity according to the electrostatic interaction. The exploration of the enhancement mechanism of graphene and improvement of the SERS performance of graphene-only substrates is only in its infancy, and much work needs to be done in the application fields of biosensors.

The synergistic effect of graphene and plasma nanomaterials greatly improves the sensitivity, and the chemical inertia and gas barrier ability of graphene endow the substrates with long-term stability. Compared with either material alone, graphene-based nanomaterials as SERS biosensors provide many unique physicochemical properties and biological applications that are ideal and obviously advantageous ${ }^{109,110}$. AuNP-graphene nanomaterials have become one of the most popular subjects among noble-based graphene SERS substrates and demonstrate remarkable surface chemical properties, high chemical stability, excellent catalytic activity, biocompatibility, and other notable properties ${ }^{110}$. Thus, AuNPs have become an ideal component for in situ detection of glucose, DNA, enzymes, and proteins; rapid identification of microorganisms; and differentiation of cancer markers from healthy individuals for prevention of major diseases ${ }^{111,112}$. On the other hand, graphene or GO has a GERS effect with larger Raman signals through the CM independent of noble metal NPs. The integrated graphene-metal hybrid enhancement effect is mainly the sum of the EM based on the local electromagnetic field and GO-based chemical mechanism of charge transfer and chemical bonding with biological molecules ${ }^{113}$. These excellent properties make graphene-Au nanocomposites an ideal substrate for SERS biosensor measurements.

A few examples of GERS biosensor platforms are summarized in Table 2. Several alternative graphenebased plasma hydride materials beyond single coinage metals have been developed, including rGO film, GO, graphene, popcorn-shaped GO, CVD-G film, graphene templates, PEGylated nanosized graphene, and porous graphene with compositions including noble metals such as $\mathrm{Au}$ and $\mathrm{Ag}$ or Raman-active semiconductors such as $\mathrm{Cu}_{2} \mathrm{O}$ or even $\mathrm{Fe}_{3} \mathrm{O}_{4}$ NPs. The effect of $\mathrm{Fe}_{3} \mathrm{O}_{4}$ nanocrystals on peroxidase activity was systematically studied. 
Table 2 Recent high-performance reports utilizing GERS biosensors of various biomolecule platforms.

\begin{tabular}{|c|c|c|c|c|}
\hline SERS substrate & Biodetection samples & $\mathrm{EF}$ & Detection limit & Refs. \\
\hline rGO film/AuNP & 4-ATP, 2-TU & $5.6 \times 10^{5}$ & $0.1 \mu \mathrm{M}(4-\mathrm{ATP}), 1 \mu \mathrm{M}(2-\mathrm{TU})$ & 239 \\
\hline G/Au nanopyramid & Dopamine and serotonin & $10^{10}$ & $10^{-10} \mathrm{M}$ & 240 \\
\hline GO/AuNP & Adenine & $1.2 \times 10^{7}$ & $10^{-7} \mathrm{M}$ & 107 \\
\hline Popcorn-shaped GO/AuNP & HIV DNA, Staphylococcus aureus & NA & $10 \mathrm{CFU} / \mathrm{m}$ & 241 \\
\hline CVD-G film/AuNP & Multiplex DNA & NA & $10 \mathrm{pM}$ & 242 \\
\hline G templates/Au nanohexagons & Cancer stem cells & NA & $100 \mu \mathrm{g} / \mathrm{L}$ & 243 \\
\hline $\begin{array}{l}\text { GO/PVP/intracellularly grown gold nanoparticles } \\
\text { (IGAuNs) }\end{array}$ & Mammalian cells: A549, 4T1, and HeLa cells & NA & NA & 244 \\
\hline PEGylated nanosized graphene/AuNP & Immunoglobulin G & $1.34 \times 10^{11}$ & $31 \mathrm{fM}$ & 245 \\
\hline \multicolumn{5}{|l|}{ Clusters (PNG-AuNPCs) } \\
\hline Au clusters@Rubpy/GO & $\begin{array}{l}\text { Ablated S. aureus and } \\
\text { E. coli bacteria, photothermal treatment }\end{array}$ & NA & NA & 163 \\
\hline GO-Au@polyaniline (PANI) & $\begin{array}{l}\text { 4T1 tumor cell imaging and drug } \\
\text { delivery of DOX }\end{array}$ & NA & NA & 246 \\
\hline $3 \mathrm{D}$ porous graphene $(\mathrm{GN}) / \mathrm{Fe}_{3} \mathrm{O}_{4} \mathrm{NPs}$ & Glucose & NA & $0.8 \mu \mathrm{M}$ & 114 \\
\hline $\mathrm{rGO} / \mathrm{AgNPs}$ & E. coli, Staphylococcus aureus & NA & $10^{5} \mathrm{CFU} / \mathrm{cm}^{3}$ & 247 \\
\hline $\mathrm{Ag}-\mathrm{Cu}_{2} \mathrm{O} /$ reduced graphene oxide $(\mathrm{rGO})$ & Glucose & NA & $10^{-8} \mathrm{M}$ & 190 \\
\hline
\end{tabular}

EF enhancement factor, 2-TU 2-thiouracil, NA not applicable.

It was found that various iron-based nanocrystals have peroxidase-like catalytic activity and can be used for peroxidase-like catalytic reactions and in situ monitoring with glucose biosensors ${ }^{114,115}$. For GERS, primitive graphene can be noncovalently functionalized by strong $\pi-\pi$ interactions between its surface and an immobilized host. In addition, $\mathrm{GO}$ or $\mathrm{rGO}$ provides additional covalent functionalization options due to the multiple oxygencontaining functional groups. To overcome the above problems related to small molecular surfactants, polyethylene glycol (PEG) was used as a surface coating agent to solubilize graphene or GO, and the end groups available at the end were used to give further surface functionalization ability. The PEG chain includes $-\mathrm{NH}_{2}$ (polyaniline) and $-\mathrm{COOH}$ groups. Biosensing ranges include biomolecules such as 4-ATP, 2-thiouracil, dopamine, adenine, and glucose; proteins such as HIV DNA, multiplex DNA, and immunoglobulin G; cells such as cancer stem cells, 4T1 tumor cells, and mammalian cells: A549, 4T1, and HeLa cells; and bacteria such as ablated Staphylococcus aureus and Escherichia coli or diagnosis of DNA damage, such as hereditary diseases, physiological disorders, and cancer cells. Herein, graphene or its derivatives play multifunctional roles: (I) as a $2 \mathrm{D}$ scaffold to fix interfacial assembled NPs; (II) as a nanospacer to generate SERS-active nanogaps between two layers of NP arrays; (III) as a collector to concentrate molecules of interest via $\pi-\pi$ interactions; (IV) as CM sources as SERS platforms; and (V) as a fluorescence quenching platform in biosensing systems. In addition, graphene could be employed as a reusable substrate since there is no specific chemical interaction between single-layer graphene and the target DNA or biomolecules. Graphene has overcome some limitations of SERS substrates because it endows SERS substrates with better stability, sensitivity, reproducibility, and biocompatibility. However, a linear correlation between the Raman peak intensity and the biodetection sample concentration or logarithm of the concentration is difficult to achieve or is only achieved in a narrow range, which greatly limits its application.

\section{Three-dimensional (3D) carbon nanomaterials and carbon-based shell-isolated NP-enhanced Raman spectroscopy (SHINERS) \\ 3D graphene/CNT composites}

Compared with 2D nanostructures, 3D structures can provide more "hot spots." These hot spots provide enormous enhancement and a larger specific surface area than 2D substrates for adsorbing probe molecules, so 3D nanostructures are far superior to $2 \mathrm{D}$ substrates ${ }^{116,117}$. Graphene-based 3D spatial SERS structures include different types of metal NPs decorating nanoporous structures, different layer coupling spatial sandwich structures between graphene and noble metal NPs, and so on. Zhang et al. ${ }^{118}$ obtained a SERS-active substrate in a new dimension that was based on GO-embedded Au@Ag-NP 
sandwich nanostructures (Au@Ag-NPs/GO/Au@AgNPs) and achieved higher sensitivity (for R6G with an enhancement factor of $7.0 \times 10^{7}$ ), reproducibility, and reliability of the Raman readout due to abundant hot spots on the surfaces and the distinctive edifice of the GO sheets. Leem et al. ${ }^{119}$ synthesized a 3D crumpled graphene-AuNP hybrid structure, which was formed by depositing Au films onto graphene grown on a $\mathrm{Cu}$ foil and then dewetting the Au films by heat treatment to form AuNPs. After that, the hybrid structure was transferred to a polystyrene substrate, and finally, 3D crumpled graphene-AuNP hybrid structures were obtained as the PS substrate shrunk when the temperature rose above its glass transition temperature. The substrate exhibits $>10$ fold higher SERS sensitivity than flat graphene-AuNPs. This simple, rapid, and ultrasensitive Raman detection method has shown great potential in the fields of food/ environmental safety monitoring and spectral identification of trace pesticides or biological molecules in in vivo systems. Kim et al. $^{120}$ began using SERS 3D GOencapsulated AuNPs to monitor in situ the undifferentiated or differentiated status or differentiation level of neural stem cells. The number of $\mathrm{C}=\mathrm{C}$ bonds is positively correlated with the Raman signal strength at $1656 \mathrm{~cm}^{-1}$. In fact, the membranes of undifferentiated cell lines contain polyunsaturated fatty acids, and the $\mathrm{C}=\mathrm{C}$ bond of undifferentiated cell lines is more abundant than that of normal/differentiated cells, which is the principle of using SERS to differentiate cells.

\section{Plasma core-shell nanostructures}

Tian and co-workers ${ }^{121}$ first provided a SHINERS mode that can prevent the SERS substrate from contacting the detection object. Graphene can offer special advantages as an NP isolating material. This kind of core-shell plasma nanostructure can provide a more realistic SERS signal than bare metal NPs ${ }^{122}$. As shown in Fig. 5, combined graphene and AuNPs can also be fabricated as shellisolated NPs called graphene-isolated AuNPs (GIAN). GIAN can be obtained by hydrophobic interactions or $\Pi$ bonding ${ }^{123,124}$. GIAN provide strong D and G vibrational mode SERS signals at $\sim 1325$ and $1595 \mathrm{~cm}^{-1}$. Either of these bands can be used for Raman imaging. In addition, GIAN are biocompatible. Due to the biocompatibility of gold NPs, GIAN can be used in biochemistry. By controlling the ultraviolet absorption peaks of GIAN to appear in the near-infrared (NIR) band, the application of functional graphene matched with biofunctional molecules such as adapters and detection cells in bioimaging and photothermal therapy can be further expanded ${ }^{125}$. Amorphous carbon materials have the advantages of acid and alkali resistance, good electrical conductivity, and good adsorption performance. Importantly, covering the plasma core with an ultrathin carbon shell can greatly improve the resistance to acids, alkalis, and other solvents. Pan Li et al. ${ }^{126}$ developed a hydrothermal route for the synthesis of Ag@C nanocomposites by changing the reaction temperature and time, and a thinner carbon shell was obtained by the improved hydrothermal method. The carbon shell thicknesses of the samples prepared at 140 for $360 \mathrm{~min}$, at $100 \mathrm{for} 80 \mathrm{~min}$, and at 60 for $60 \mathrm{~min}$ were $\sim 2.5,1.5$, and $1 \mathrm{~nm}$, respectively.

SHINERS has changed the working mode from direct contact (SERS) or noncontact (tip-enhanced Raman scattering, TERS) to shell isolation. It breaks some longstanding limitations, allows characterization of chemical systems involving previously inaccessible surface materials and morphologies and simplifies analysis in challenging environments such as biological systems. The use of ultrathin chemically and electrically inert shells around nuclear power sources can solve many problems ${ }^{127}$. It prevents contact with the chemical environment, with surfaces of interest, and with probe molecules. It can also maintain excellent stability by preventing the NPs from aggregating $^{128}$. In addition, GIAN show two-photon luminescence. The advantages of two-photon luminescence imaging include minimal autofluorescence background, minimal photon-induced damage to the sample, and high spatial resolution ${ }^{129,130}$.

As an example of DNA detection (Fig. 7 and Table 3), Duan et al. ${ }^{131}$ developed an efficient modularization method for SERS substrates based on the chemical derivation of GO-thiolated $\mathrm{GO}$ ( $\mathrm{tGO}$ ) nanosheets gapping two layers of Au@Ag NP arrays (Au@Ag-tGO-Au@Ag) and an interfacial assembly technique using plasma NPs as templates to detect multiple DNA sequences of bacterial pathogens simultaneously. They employed three different Raman dyes with distinct spectral fingerprints for tagging single-stranded DNA. Specifically, HEX-, Cy5, and TAMRA-encoded reporter DNAs were used to detect the gene sequences of E. coli O157:H7, S. aureus, and Listeria monocytogenes, respectively. Moreover, the highly sensitive "signal-off" detection method could detect the DNA sequences of E. coli O157:H7 down to $10 \mathrm{pM}$ using HEX-labeled reporter DNA as the SERS probe. Herein, tGO has many functions: (1) as a 2D scaffold of $\mathrm{Au} @ \mathrm{Ag}$ NPs assembled at a fixed interface; (2) as a nanospacer to generate SERS-active nanogaps between two layers of NP arrays; and (3) as a collector to enrich molecules of interest through $\pi-\pi$ interactions. The modular nature of the strategy and the latest developments in wet chemical synthesis of plasma nanocrystals provide new opportunities to obtain basic insights into the relationship between SERS performances.

The development of ultrathin pinhole-free shell materials with unique functions, especially $2 \mathrm{D}$ materials such as graphene, is a promising direction for the study of novel core-shell NP-enhanced Raman spectroscopy. 

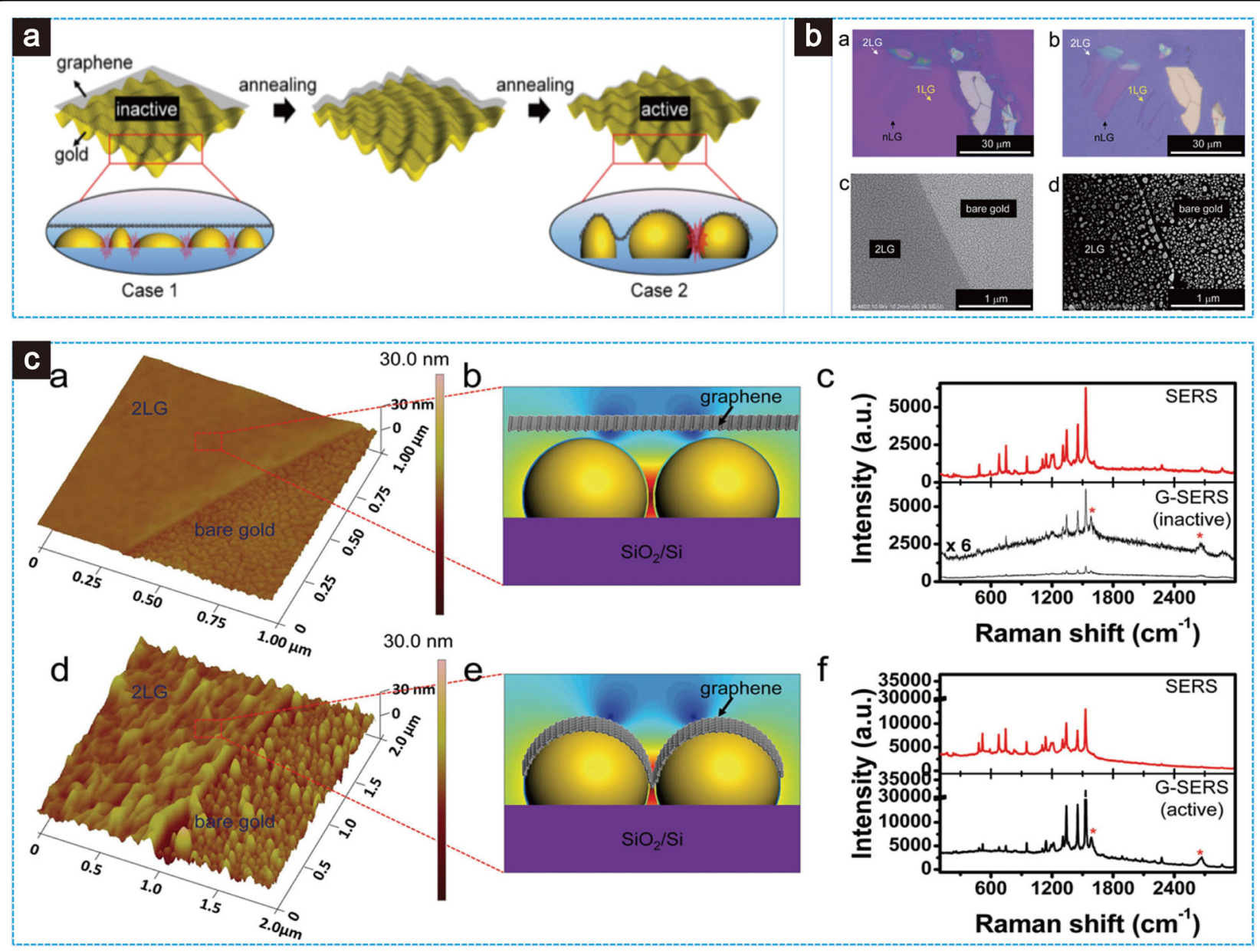

Fig. 5 Graphene-isolated AuNPs (GIAN) SERS sensors. a Schematic illustration of graphene-veiled SERS substrates with graphene spread over the curved side of Au nanoislands in two cases, which exhibit distinct electromagnetic enhancement activity. Here, an 8-nm film of Au nanoislands was used as the electromagnetic enhancer. Case 1 (SERS inactive) and case 2 (SERS active) are the cases before and after a thermal annealing activation process, respectively. $\mathbf{b}$ Optical and SEM images of exfoliated graphene on an $\mathrm{Au}_{\mathrm{S}} \mathrm{SiO}_{2} / \mathrm{Si}$ substrate before and after annealing: $\mathrm{a}$, b) Optical images of graphene on an 8-nm gold film a) before, and b) after annealing. Single layer (1L-), bilayer (2L-), and few-layer (nL-) graphene pieces are marked with arrows. C, d) SEM images of a 2LG on an 8-nm gold film c) before, and d) after annealing. c Morphology of graphene-mediated SERS (G-SERS) core-shell plasma nanostructures: (a, d) atomic force microscopy images of a bilayer graphene (2LG)-covered 8-nm gold film (a) before and (d) after annealing, showing both the bare gold regions and graphene-covered regions; $(b, e)$ schematic illustration of the contact state between graphene and AuNSs corresponding to the enlarged regions; (c, f) SERS performance of normal SERS (top) and G-SERS (bottom) regions (c) before and ( $f$ ) after annealing ${ }^{158}$. Copyright@ 2013, Wiley.

Core-shell NPs for SERS can also be used in the field of SERS biosensors. To provide a clear view of this field for readers, various systems of the SHINERS biosensor platform and their applications in SERS are summarized in Table 3. According to the enhancement mechanism and source of the SERS signal, the core-shell NPs in SERS can be roughly divided into two categories: ultrathin coreshell NPs and thick core-shell NPs. NPs with ultrathin shells mainly enhance the Raman signals of molecules on or near the surface of the shell by means of strong electromagnetic fields of SERS-active nuclei, while NPs with thick shells are usually accompanied by multifunctional extended SERS applications. Ultrathin shells can be divided into metal shells and nonmetal shells. Metal enclosure refers to transition metal enclosure, such as $\mathrm{Pt}$, $\mathrm{Pd}$, and $\mathrm{Ru}$. Nonmetal shells refer to the silica shell, $\mathrm{Al}_{2} \mathrm{O}_{3}$ shell, $\mathrm{TiO}_{2}$ shell, graphene shell, carbon (C) shell, and other oxide shells. Therein, graphene has a larger Raman cross-section and a more stable structure. The strong bands at 1325 and $1596 \mathrm{~cm}^{-1}$ are attributed to the D-band and G-band of graphene, respectively. Both the D-band and G-band can be used in the imaging unit. Therefore, graphene is a suitable candidate for cell imaging. In addition, the shell of graphene can be modified by functional groups such as PEG molecules so that the hydrophobic shell can become a hydrophilic surface and exhibit improved biocompatibility. Amorphous carbon materials have the advantages of acid and alkali resistance, good 
Table 3 Recent high-performance reports utilizing SHINERS biosensors of various biomolecule platforms.

\begin{tabular}{|c|c|c|c|c|}
\hline SERS substrate & Biodetection samples & $\mathrm{EF}$ & Detection limit & Refs. \\
\hline CNT/SiO $2 @(A u / A g)$ & Adenine & NA & $10^{-4} \mathrm{M}$ & 248 \\
\hline \multirow[t]{2}{*}{ TriplexAu@Ag@C core-shell NPs } & Quantitative immunoassays of mouse lgG & NA & $100^{-10} \mathrm{ng} / \mathrm{mL}$ & 133 \\
\hline & $\begin{array}{l}\text { Nanotoxicology test in living Nicotiana benthamiana } \\
\text { plants and biochemical imaging }\end{array}$ & NA & NA & 134 \\
\hline Au@nanosize GO (NGO) & HeLa cell bioimaging and anticancer drug DOX delivery & NA & NA & 249 \\
\hline Au@AgNP-MWCNT hybrid & HeLa cell imaging & NA & NA & 250 \\
\hline $\begin{array}{l}\text { G-isolated-Au- } \\
\text { nanocrystal (GIAN) }\end{array}$ & $\begin{array}{l}\text { Loading anticancer drug doxorubicin (DOX) for therapy, } \\
\text { multimodal cell imaging }\end{array}$ & NA & NA & 132 \\
\hline $\mathrm{M} @ \mathrm{G}, \mathrm{M}=\mathrm{Cu}, \mathrm{Ag}$, and $\mathrm{Au}$ & Photobleaching and fluorescence quenching of CoPc & NA & NA & 130 \\
\hline Au@Ag-tGO-Au@Ag & $\begin{array}{l}\text { Multiple DNA sequences and gene sequences of E. coli } \\
\text { O157:H7 }\end{array}$ & $2.7 \times 10^{8}$ & $10 \mathrm{pM}$ & 131 \\
\hline Ag/PATP@SiO $/$ /CND & Immunoassay detection of goat-anti-mouse lgG & NA & $\begin{array}{l}0.0025 \mu \mathrm{g} / \mathrm{mL} \text { (quantitative analysis: } \\
0.01-100 \mu \mathrm{g} / \mathrm{mL} \text { ) }\end{array}$ & 238 \\
\hline GO-Au@PANI & $\begin{array}{l}\text { Anticancer drug DOX delivery, NIR imaging of } \\
\text { cancer cells }\end{array}$ & NA & $1 \mu \mathrm{g} / \mathrm{mL}(\mathrm{DOX})$ & 246 \\
\hline GO@SiO & Glucose & NA & $2 \mathrm{mM}$ & 188 \\
\hline
\end{tabular}

$N A$ not applicable, EF enhancement factor.

electrical conductivity, and good adsorption performance. Importantly, covering the plasma core with an ultrathin carbon shell can greatly improve the resistance to acids, alkalis, and other solvents. Generally, there are two preparation methods: chemical methods and physical methods. Reflectors of different sizes, shapes, and materials can be fabricated to meet different requirements or applications. As summarized in Table 3, for example, because of the strong electromagnetic field from the Au core and the chemical enhancement from the graphene shell, with GIAN $^{132}$, the Raman signal can be greatly enhanced and has been widely used in biomedical research. In addition, a controllable and convenient hydrothermal synthesis method was reported that can combine Raman marker molecules with $\mathrm{Au}-\mathrm{Ag}-\mathrm{C}$ core-shell NPs layer by layer. 4Mercaptobenzoic acid is a new Raman marker for quantitative immunoassays ${ }^{133}$. Interestingly, in their follow-up study, the authors also used the triplex $\mathrm{Au}-\mathrm{Ag}-\mathrm{C}$ in a unique method to deliver carbon-encapsulated SERS tags into the leaves of Nicotiana benthamiana. As a noninvasive and nontoxic biochemical imaging method for living plants, the interference of self-fluorescence of plant tissues was successfully avoided ${ }^{134}$.

\section{SERS biosensors}

\section{Principles and SERS hot spots}

SERS is a Raman spectroscopic technique that can provide highly enhanced Raman signals from Ramanactive analytes adsorbed on the surface of nanostructures.
The enhancement factor strongly depends on the size, shape, and composition of SERS nanostructures and the properties of molecular analytes. The SERS effect is caused by two different mechanisms: the EM and CM. In particular, the application of carbon-based materials as Raman-active substrates has attracted increasing attention because it provides a platform for distinguishing two known entanglement-prone enhancement mechanisms (EM and CM) in SERS, as mentioned above. The main contributor to most SERS processes is the EM. The enhancement is caused by light amplification excited by local surface plasmon resonance (LSPR). The working principles of SERS are depicted in Fig. 6. LSPR from plasma nanomaterials can be excited by far-field incident light and can focus the light on the edge, tip, or gap on the nanoscale, thus increasing the local electromagnetic field intensity by two to five orders of magnitude. LSPR can also be excited by a local oscillator (such as a dipole or quadrupole) and directionally reradiated into the far field (Fig. 6a, b). This light concentration occurs due to the existence of "hot spots" on the SERS-active structure located preferentially in the NP gaps, in the crevices, or at the sharp features of plasma materials, which are traditionally noble and coinage metals (e.g., silver, gold, and copper) with nanoscale features, and SERS signals can reach an enhancement of $10^{13}-10^{15}$ times $^{117,135-138}$. The $\mathrm{CM}$ is usually caused by electron transfer between surfaces such as graphene and adsorbed molecules, which is usually limited to a $10-10^{2}$ enhancement ${ }^{138,139}$. 

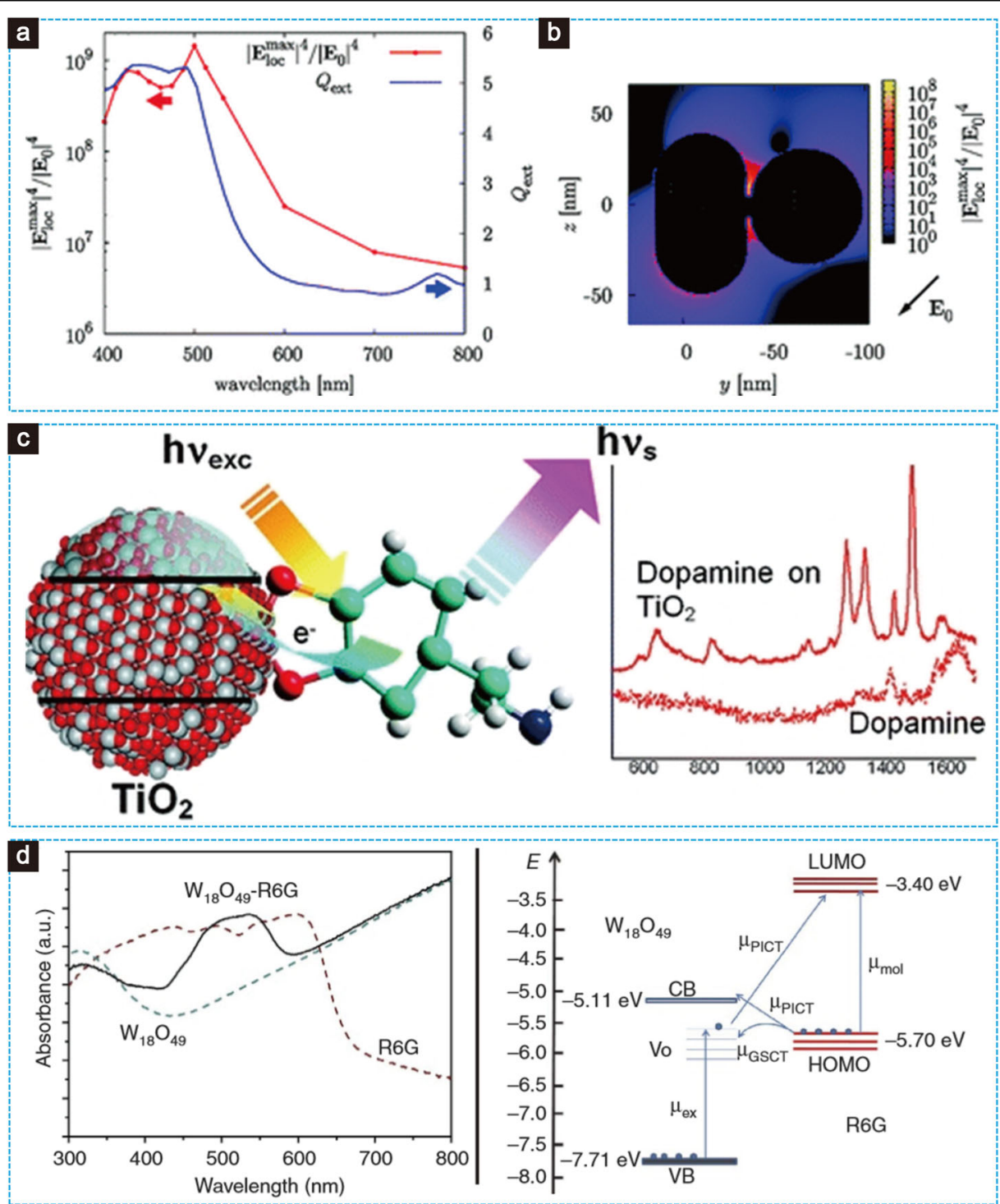

Fig. 6 Enhancement mechanisms of both EM and CM in SERS. a, b Wavelength-dependent extinction efficiency $\left(Q_{\mathrm{ex}}\right)$ and local values of $E_{\mathrm{EM}}$, calculated as $\left|E_{\text {loc }}\right|^{4} /\left|E_{0}\right|^{4}$ using the discrete-dipole approximation (DDA) of an Ag nanocrystal contact and the hot spot location distribution at the cusp between particles ${ }^{251}$. Copyright@ 2008, American Chemical Society. c Typical CM effect schematic illustration, and SERS spectra of various biomolecules on a small-size $(5 \mathrm{~nm})$ titanium dioxide surface ${ }^{140}$. Copyright $\odot 2009$, American Chemical Society. d Charge-transfer study between R6G and $\mathrm{W}_{18} \mathrm{O}_{49}$ : absorption spectra for $\mathrm{R} 6 \mathrm{G}$ on $\mathrm{W}_{18} \mathrm{O}_{49}$ compared with neat $\mathrm{W}_{18} \mathrm{O}_{49}$ and $\mathrm{R} 6 \mathrm{G}$ dye, and energy level diagram of R6G on oxygen-deficit $\mathrm{W}_{18} \mathrm{O}_{49}$ measured in a vacuum ${ }^{141}$. Copyright@ 2015, Springer Nature.

The electromagnetic field on the surface of plasmon polaritons is highly localized in the hot spot range and is not uniformly distributed. Hot spots can be nanoneedles or nanogaps between particles or between particles and substrates. As summarized by Tian et al. ${ }^{17}$, hot spotnanoscale regions with a strongly enhanced local electromagnetic field have undergone three generations of development: (I) The first-generation SERS hot spots are generated from single nanostructures-when nanospheres, nanocubes or nanorods are dispersed in a homogeneous medium, a single nanostructure can produce hot spots. Generally, these hot spots exhibit moderate SERS activity. However, some specially designed particles with a sharp structure can exhibit higher SERS activity, such as stellate or flower-like AuNP and AgNP. (II) The second-generation SERS hot spots are generated by coupled nanostructures with controllable intergranular or interunit nanogaps. This kind of hot spots produced by the nanoscale gap between oligomers or nanoscale arrays shows excellent SERS activity, and the average intensity of a single nanoscale structure is $2-4$ orders of magnitude, which can be used to detect a single 
molecule. (III) The third-generation SERS hot spots are generated by hybrid structures consisting of plasma nanostructures and probe materials. Generally, first- and second-generation hot spots are not suitable for surface analysis of many materials. For example, materials such as silicon and ceramics simply cannot enter nanogaps. Therefore, it is necessary to design and develop plasma nanostructures that can directly realize hot spots on the surface of materials. Considering that LSPR and the local electric field are not only dependent on the size and morphology of the plasma structure but also influenced by the dielectric properties of the materials to be measured near the nanostructures, the third-generation hot spots in complex nanostructures should be transferred to the surface of the materials to be measured.

In 2009, Musumeci et al. ${ }^{140}$ reported the SERS spectra of various biological molecules on the surface of smallsize $(5 \mathrm{~nm}) \mathrm{TiO}_{2}$ (Fig. 6c) and demonstrated the charge transfer between $\mathrm{TiO}_{2}$ and the adsorbed molecules, which is the reason for the SERS effect of NPs. The enhancement factor was estimated to be $\sim 10^{3}$. This is an important research progress in the CM of SERS. It is worth noting that nonstoichiometric tungsten oxide nanostructures, similar to the $\mathrm{W}_{18} \mathrm{O}_{49}$ nanowires of sea urchins, were used as substrate materials to amplify the interaction between the base analyte and molecule (Fig. 6d), resulting in significant changes in the Raman spectra. The enrichment of surface oxygen vacancies can lead to additional enhancement $^{141}$. The detection limit is as low as $10^{-7} \mathrm{M}$, and the maximum enhancement factor is $3.4 \times 10^{5}$. Semiconductor materials can even be comparable to noble metals without "hot spots" by forming oxygen vacancies.

To help readers understand the design principles of carbon-based SERS substrates as biosensors more clearly, a comparison between carbon-based SERS substrates and other carbon-free SERS substrates is necessary. SERS substrates with excellent surface uniformity and enhancement efficiency in terms of both carbon-free and carbon-based SERS substrates have been well developed recently. For carbon-free SERS substrates, three representative platforms are commonly included: the traditional gold-standard Klarite substrate fabricated by topdown lithography or an electrochemical method, monodispersed or aggregated colloidal plasma NP-based SERS in solution, and a recent new soft 2D SERS substrate fabricated by self-assembly. The aggregation of metal colloids may be the easiest way to construct substrates that exhibit enormous field enhancement for ultrasensitive or even single-molecule SERS detection ${ }^{142,143}$. The most commonly used Au and Ag colloids are generally prepared by one-step or multistep chemical reduction methods ${ }^{144-146}$ and are usually stabilized by citric acid in solution with reasonable size or shape control and monodispersity. Aggregation of these colloids can be achieved by the addition of a salt (charge shielding), allowing van der Waals attraction between particles to dominate the system ${ }^{144}$. For example, Ji and coworkers ${ }^{147}$ proposed a novel method of using a highly sensitive SERS sensor for the detection of hydrophobic analytes based on cetyltrimethylammonium bromide (CTAB)-triggered Ag aggregates. The introduction of CTAB into Ag colloids can significantly improve the binding ability of AgNPs to hydrophobic analytes. At the same time, CTAB can trigger the formation of $\mathrm{Ag}$ aggregates, forming a large number of SERS-enhanced hot spots. Compared with the monodisperse sol system, the colloid aggregation of metal NPs can achieve great EM due to the formation of a large number of hot spots, thus further reducing the detection limit. However, their formation was limited by the randomness, which led to a great change in the field enhancement and SERS intensity over the whole substrate. In addition, the random clustering method lacks repeatability of the substrate, which makes it difficult to optimize more quantitative SERS measurement conditions.

Compared with single-element particles, bimetallic NPs provide a richer plasma pattern due to their combination of material and size/shape-dependent plasmons. In particular, core-shell NPs have recently been widely used as effective SERS sensors. For the design strategy of carbonfree core-shell SERS sensors, which are termed SHINERS substrates, the clever introduction of a thin shell prevents particles from aggregating, as discussed above, prevents contaminants from adsorbing onto the metal surface, and allows particles to spread on any type of surface and even form a SERS substrate at the top of a battery. Although the shell reduces the maximum enhancement due to the distance between the analyte and the metal surface, the greater reproducibility of the Raman strength makes the technique more quantitative and reliable. More recently, size- and shape-dependent monodisperse $\mathrm{Au} \mathrm{Ag}$ nanocubes (NCs) and Au@Ag NBs were developed by Cheng's group ${ }^{148}$, in which the Ag shell thickness could be precisely adjusted from $\sim 1$ to $\sim 16 \mathrm{~nm}$ (Fig. 7a). This work expands the morphology range of the SHINERS structure to nanocubes or NBs, so the morphology is no longer limited to nanospheres, and reveals that the excitation wavelength distinguishes the shape of the particles. The maximum Raman enhancement is observed when the excitation wavelength matches the SERS peak, on the premise that there is an obvious local electric field limitation in the plasma nanostructure at this wavelength. The Raman enhancement reaches the maximum at a certain threshold of particle size (or silver coating thickness). By optimizing the particle size, the excitation at the corresponding laser wavelength is optimized. According to this mechanism, the detection limit of the sensor can 

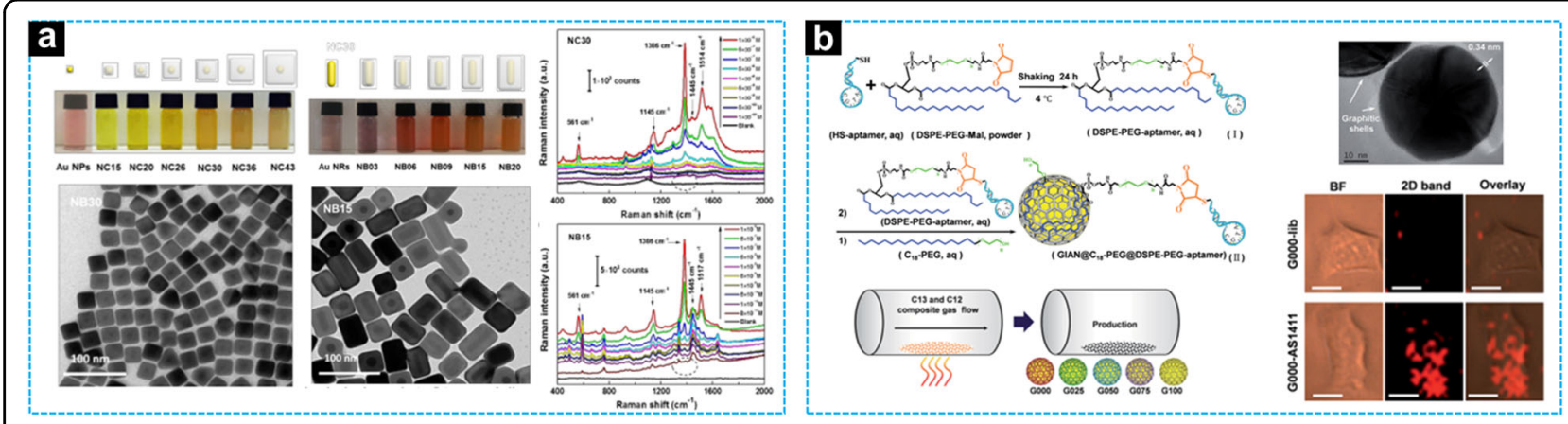
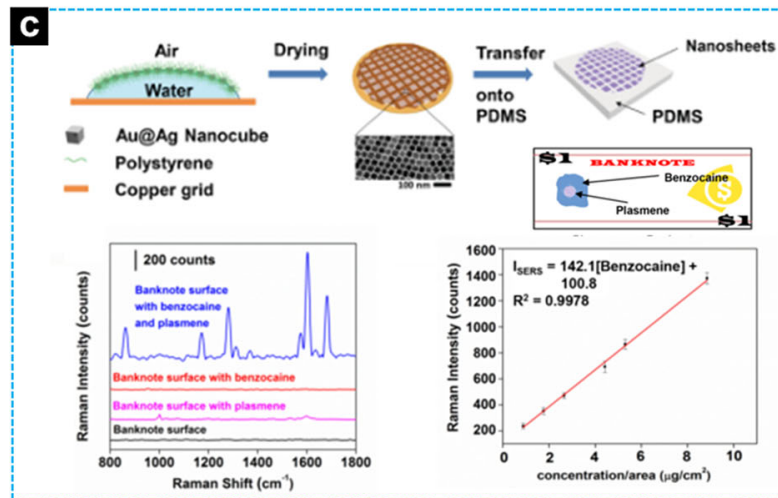

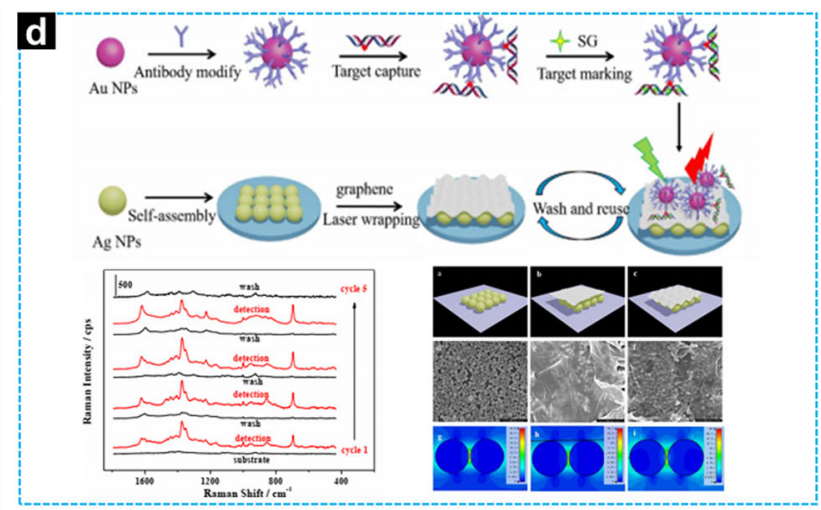

Fig. 7 Comparation of traditional Klarite SERS substrates and novel developed carbon-based SERS biosensors. a Typical traditional Klarite SERS substrates without carbon-based nanomaterials of monodisperse Au@Ag nanocubes (NCs) and Au@Ag nanocuboids (NBs) in colloidal solutions, which are designed as size/shape-dependent core-shell SERS substrates, with a finely tunable Ag shell thickness and a pM concentration detection limit as a sensitive chemical sensor ${ }^{148}$. Copyright $\odot$ 2015, Royal Society of Chemistry. b Novel developed carbon-based core-shell SERS substrates of multiplexed isotopic GIAN with cellular Raman-silent signals for cancer cell pattern recognition ${ }^{149}$. Copyright@ 2018, Royal Society of Chemistry. c Recent new soft 2D plasmene nanosheet substrates of Au@Ag NCs as model building blocks without carbon nanomaterials, which served as a new class of SERS substrates that offer unique capabilities of direct surface attachment and direct quantitative drug identification on surfaces without the need for additional processing steps ${ }^{153,154}$. Copyright@ 2015, Wiley and Copyright $\odot$ 2015, American Chemical Society. d Typical carbon-based 2D reusable laser-wrapped graphene-Ag array SERS biosensor for trace detection of genomic DNA methylation, and corresponding theoretical considerations for the FDTD simulation of coating carbon materials onto metallic particles ${ }^{157}$. Copyright@ 2016, Elsevier.

be further optimized. For carbon-based SERS core-shell biosensors, GIAN possess their own unique application advantages. For example, Chen and co-workers ${ }^{149}$ proposed SERS-encoded NPs with a stable and simple fingerprint spectrum according to the synthesis of isotopic cellular Raman-silent GIAN and conjugation with phospholipid-PEG-linked aptamers to target proteins overexpressed on the cancer cell surface (Fig. 7b). GIAN have the superstable optical advantage of graphene nanomaterials and a high Raman cross-section, showing high SERS signals. Such GIAN tags show a multiplex Raman imaging ability both in vivo and in vitro, and the background interference is very small. In addition, aptamer-conjugated isotopic GIAN have cell membrane protein (nucleolin, mucin, and epithelial cell adhesion molecule) specificity and have been successfully applied to built-in coding for rapid imaging and pattern recognition of targeted cancer cells. This isotopic GIAN aptamer encoder shows high potential in the effective identification and diagnosis of cancer cells. As a whole, carbon-based core-shell SERS substrates show higher potential and better flexibility as biosensors for cost-effective cancer cell or in vivo system identification with high sensitivity and low background interference, promising a novel tool for more biomedical applications.

To overcome the problems of poor repeatability and uniformity in colloids, especially aggregated SERS substrates, 2D SERS substrates have rapidly been developed in recent years. 2D SERS substrates with improved uniformity have also been prepared mainly by controlled deposition and lithographic and template synthesis strategies. Colloid NPs are assembled onto surfaces by polymers with a functional anchoring group, such as mercaptan or amine. The use of coordination layers can better control the spacing between particles, which further controls the hot spots. Because of the high controllability and reproducibility, photolithography and related template methods are usually used to fabricate SERS substrates with precise adjustable hot spots, even to produce gaps of one to several nanometers according to 
adjustment of experimental conditions. These periodic SERS arrays allow tailoring of their structures and arrangements according to the application, whether for experiments on the basic physics of single molecules or large-scale production of SERS sensors. At present, various lithography/template techniques have been reported for the preparation of high-quality SERS substrates, including nanowire templating, nanosphere lithography, line lithography, electron beam lithography, and nanotransfer techniques ${ }^{142,150-152}$. A new class of ultrathin SERS substrate-soft plasmene nanosheets was fabricated using a simple yet efficient bottom-up self-assembly approach by Chen and co-workers ${ }^{153,154}$. These nanosheets are ultrathin, mechanically elastic, and optically translucent and can be attached to complex topological surfaces in the real world (banknotes and plastic banknotes, even metal coins), achieving repeatable and sensitive detection of chemicals (see Fig. 7c). Different from traditional rigid SERS substrates, this flexible 2D substrate can be used to directly identify drugs and achieve a benzocaine-sensitive detection limit of a few parts per million (p.p.b.) with an excellent linear relationship $\left(R^{2}>\right.$ 0.99). On the surface of banknotes, the detection limit of benzocaine reached $0.9 \times 10^{-6} \mathrm{~g} / \mathrm{cm}^{2}$. In addition, several other drugs can be identified even in binary mixtures by the plasma membrane nanosheet sensor. In the design of carbon-based 2D SERS substrates, the specificity and sensitivity for biomolecules can be analyzed by the functional design of their surfaces ${ }^{135,155,156}$. Recently, Ouyang et al. ${ }^{157}$ developed a laser-wrapped graphene-Ag array as an effective specific SERS biosensor (see Fig. 7d) to detect trace genomic DNA methylation as an indicator for the initiation and progression of cancers among other diseases by making use of methylation-specific antibodymodified AuNPs as the target scaffold to enrich methylation-related DNA and a laser-wrapped grapheneAg array as an enhancing substrate. Herein, due to the effective coupling of the electromagnetic field, the graphene layer can enhance the SERS signal. In addition, the graphene wrapping approach circumvents the direct interaction between the Ag array and the analytes, thus improving the reusability of the SERS substrate even after five cycles of use. This method has good clinical application prospects, especially when combined with portable instruments. Information on global methylation will provide the basis for specific gene research with this kind of carbon-based novel SERS biosensor.

Theoretical considerations for coating carbon materials onto the surfaces of metallic particles or arrays for SERS applications are necessary for an essential understanding of the material design. For example, as shown in Fig. 7d, the ultrathin graphene layer on the $\mathrm{Ag}$ nanoarray enhances the Raman signal due to the effective transfer of the electromagnetic field from the metal core to the probe molecule through the shell. The electromagnetic field distributions of the Ag array before and after graphene coating are simulated by FDTD, as shown in Fig. 1g, h, i. The results show that when graphene is coated, the maximum electromagnetic field at the node increases by 1.3 times (based on the simulated electromagnetic field distributions), and after laser impact on graphene, the maximum electromagnetic field at the junction increases by 1.2 times. The tight coverage of the graphene monolayer leads to enhancement of the electromagnetic field of the silver array, and the electromagnetic field is more limited to the narrower region of the grapheneencapsulated Ag array, which leads to more effective enhancement at the junction between the AgNPs and an increase in hot spots, thus realizing overall enhancement of SERS activity. This enhancement is possible because the coupling of the electromagnetic field is enhanced, which well matches the SERS results, and the electromagnetic field is more limited to the narrower region of the graphene-coated Ag array, which leads to a more effective enhancement at the junction between the AgNPs. The graphene coating method avoids direct interactions between the Ag array and the analyte, thus improving the reusability of the SERS substrate. In addition, according to Zhang and co-workers' research, the mechanism of G-veiled Au arrays as effective SERS substrates was discussed in detail by using Mie theory and the 3D-FDTD simulation method ${ }^{158}$. The results (see Fig. 5c) show that the thin graphene layer does not significantly attenuate the enhanced electromagnetic field between $\mathrm{Au}$ nanostructures. They predicted that the low current activity was due to the hot spots of SERS activity being "buried" under the far graphene veil. This is also consistent with the atomic force microscopy observations. It should be noted that although it is claimed that the conductive graphene layer suppresses the surface electric field, this is not considered to be the reason for the weak SERS activity of the inactive G-SERS substrate, as it can be further thermally activated (which does not change the conductivity of graphene). Different from the SERS substrates on which most molecules are directly adsorbed on the active metal surface, this new G-veiled substrate provides a passivated surface for SERS. The graphene veil makes the SERS analysis have a clearer molecular interaction so that the enhanced Raman signal has better repeatability. This work provides a unique strategy for designing SERS substrates with the lowest loss of SERS activity after surface passivation. Furthermore, a potential enhancement mechanism of the trinary $\mathrm{AuNP} / \mathrm{MoS}_{2} / \mathrm{G}$ van der Waals heterostructure is demonstrated to be the combination of the electromagnetic mechanism of the plasma AuNPs and the CM by density functional theory calculations ${ }^{159}$. The $\mathrm{CM}$ of the $\mathrm{AuNP} / \mathrm{MoS}_{2} / \mathrm{G}$ van der Waals heterostructure is enhanced by enhancing the 
interface dipole-dipole interaction rather than by $\mathrm{G}$ or $\mathrm{MoS}_{2}$ alone. 2D nanomaterials ${ }^{160}$ exhibit a low plasma effect in the excitation wavelength range of Raman spectroscopy (ultraviolet-visible-NIR). When they are used as SERS substrates, they exhibit an obvious CM effect $^{98,105,161,162}$. In addition to the charge-transfer and dipole formation effects, some special structural properties of 2D nanomaterials, including carbon nanomaterials, can also improve the sensitivity of SERS sensors, such as the best enhancement effect of graphene monolayers compared to few or more layer graphene, which needs to be taken into account ${ }^{163,164}$. For example, the large specific surface area of $2 \mathrm{D}$ nanomaterials and the $\pi-\pi$ stacking interaction can promote the adsorption of probe molecules on 2D nanomaterials and the charge transfer between probe molecules and 2D nanomaterials ${ }^{165-167}$. The synergistic effect of each component includes improved sensitivity, stability and reproducibility, and fluorescence quenching ${ }^{75,157,168,169}$. Therefore, all these factors facilitate the broad application prospects of these materials in the fields of optoelectronics and biosensors.

\section{Surface functionalization of carbon nanomaterials for biointerfaces}

To obtain graphene-based platforms for biological applications, it is necessary to functionalize them with special elements so that graphene does not exhibit considerable biological toxicity and has functional groups that can bind with other molecules. Therefore, surface functionalization is very important for biological applications by improving the surface bioanalytical performance (such as sensitivity, biocompatibility, carrying capacity, or specificity) to make it an ideal matrix for the synthesis and design of multicomponent platforms. To date, various chemical molecules have been used to prepare functional systems integrated with small molecules, nucleic acids, peptides, proteins, and NPs by covalent and/or noncovalent methods.

The rich oxygen functional groups on the surface of GO can be used as a chemical handle for the conjugation of functional molecules by covalent bonds ${ }^{107,170}$. The carboxyl groups on GO react with the amino groups of molecules or proteins through carbodiimide chemistry, which finally disrupts the extended $\pi$-conjugation, termed "chemisorption." Common functional groups that contribute to chemisorption include mercaptan (e.g., sulfurized nucleic acid) and mercapto (e.g., found in cysteine residues of proteins) ${ }^{171}$. According to the position of these functional groups, specific regions of biomacromolecules can be covalently fixed to the surface of SERS sensors, while other bioactive regions can participate in binding interactions ${ }^{172}$. Unexpectedly, noncovalent functionalization does not disrupt the extended $\pi$-conjugation of graphene surface as covalent functionalization does, and the large 2D aromatic surface of graphene makes it an ideal matrix for adsorbing some biological molecules, such as via $\pi-\pi$ stacking interactions. GO is highly negatively charged and can adsorb positively charged molecules by electrostatic, van der Waals, or hydrophobic interactions to enhance the physical adsorption. QD-antibody complexes $(\mathrm{QD}-\mathrm{Ab})$ can also be adsorbed by $\pi-\pi$ stacking and hydrogen bonding interactions ${ }^{173}$. In this case, the coating process is simple, but the interaction between biomacromolecules and the sensor surface is weak (noncovalent), which can lead to surface-induced denaturation and/or conformational changes, thereby altering the biological activity (e.g., the recognition ability of adsorbed species $)^{174}$. For detection applications, these features are not conducive to fixing receptors on the sensor surface because they often damage the function, so this modification strategy may not meet key measurement needs, such as selectivity and stability.

\section{SERS biosensing strategies}

The general design of an LSPR sensing platform entails the choice of the (i) plasma material, (ii) geometrical properties of the nanostructures (typically NPs), (iii) fabrication method, and (iv) surface coating. A typical SERS tag consists of four parts: a SERS-active nanoscale substrate, an organic Raman reporter, a protective shell, and the target molecule ${ }^{175}$. Different kinds of SERS-active nanostructures can provide a strongly enhanced Raman spectrum generated by the local light field on the substrate surface, which is capable of being controlled by the chemical composition, size, and structure and greatly affects the SERS properties of the tags. However, this simple SERS-active nanoscale substrate-Raman reporter structure usually lacks stability, and the signal is easily disturbed by surrounding interference. Hence, carefully designed coating materials (such as core-shell structures) and treatment procedures are necessary for improving the tag biocompatibility and reducing nonspecific binding aggregation. Finally, adding targeting molecules to the SERS tags imparts biofunctionality. In terms of toxicity, when carbon-based SERS biological probes are injected into cells or in vivo for clinical trials, local administration is generally used to reduce systemic toxicity, such as by directly spraying antibody-coupled F-SERS dots on tumors, thus bypassing the main disadvantage of intravenous injection: systemic toxicity caused by accumulation in major organs (lung, liver, and spleen) ${ }^{176,177}$. Because of the large size of antibody-coupled F-SERS dots, tumor targeting is reduced. In addition, introducing a silicon shell (nontoxic coating) is well known to be an effective way to reduce the toxicity of NPs. In addition, antibody-coupled F-SERS dots do not accumulate in the normal colon after washing, thus further reducing any toxicity to normal organs ${ }^{59,178}$. It has been reported that 
some coupling agent-bound nanocomposites have cytotoxicity, such as ethanolamine-coated $\mathrm{AgNPs}^{54}$. To overcome this problem, the sulfhydryl bond between several biocompatible - $\mathrm{SH}$ groups is generally used to replace ethanolamine as the end-capping agent of the label, which can minimize the toxicity problem when applied clinically.

As shown in Fig. 8a, a ratiometric SERS sensor based on the internal standard method for sensitive and repeatable quantitative detection of microcystin-leucine arginine (MC-LR) was developed. Gap-tethered SERS-active $\mathrm{Au} @ \mathrm{AgAu}$ NPs were successfully prepared, and the gap size was adjusted by simply adjusting the acidity. These NPs showed a gap-adjustable enhancement of SERS activity and were used as a SERS tag. $\mathrm{GO} / \mathrm{Fe}_{3} \mathrm{O}_{4} \mathrm{NPs}$ exhibit unique and stable Raman bands from the graphite components, making them an ideal material for quantitative Raman analysis. In principle, the assembly of $\mathrm{Au} @ g a p @ A g A u$ NPs and GO/ $/ \mathrm{Fe}_{3} \mathrm{O}_{4}$ NPs depends on the $\pi-\pi$ stacking interaction between GO and MC-LR aptamers. In the presence of MC-LR, Au@gap@AgAu NPs and MC-LR NPs are separated due to the affinity of aptamers, resulting in changes in the SERS-labeled Raman intensity. Quantitatively, after internal standard Raman signal correction, the limit of detection (LOD) of MC-LR is as low as $9.82 \mathrm{pm}$. This strategy provides a simple and fast method for sensitive and quantitative detection of MC-LR and has broad application prospects in complex systems.

To develop a high-throughput and easy-to-operate homogeneous virus detection method, semiconductor QDs may overcome some functional limitations of protein arrays and suspension arrays due to their unique optical properties. Recent advances in QD-based biosensors have opened up new possibilities for multiple detections of proteins or viruses. To generate a fluorescence "on" detection strategy, GO was first selected as a superquencher for QD fluorescence. As shown in Fig. 8c, a new detection strategy was designed, in which the EV71 antibody (Ab1) and CVB3 antibody (Ab2) are labeled with different color QDs to realize simultaneous detection of multiple viruses. Ab1 and Ab2 are first labeled with biotinylating reagents. Streptavidin-conjugated green QDs $(525 \mathrm{~nm})$ and streptavidin-conjugated red QDs $(605 \mathrm{~nm})$ are coupled with Ab1 and AB2 via biotin-streptavidin interactions, respectively. The fluorescence of these $\mathrm{QD}-\mathrm{Ab}$ bioconjugates (QD-Ab1 and $\mathrm{QD}-\mathrm{Ab} 2)$ is quenched by GO to produce virus capture probes. Once the virus capture probe is exposed to the target of EV71 and CVB3, green and red fluorescence will be activated by the release of $\mathrm{QD}-\mathrm{Ab}$ due to the binding of antibody to antigen. If only one target exists, then the corresponding colored QDs will be released and emit strong fluorescence, indicating that the corresponding target virus exists (i.e., when EV71 exists alone, only green fluorescence emission is turned on).

SERS is a real label-free technology that directly provides intrinsic SERS signals of biomarkers of interest in complex biological matrices without any labeling or special treatment of SERS substrates and samples. These advantages make SERS an ideal alternative for sensitive and accurate detection of serum bilirubin. Logically speaking, plasma substrates are the key to obtaining high performance for SERS biosensors. In recent decades, the development of SERS biosensor technology has focused on innovating plasma nanostructures with high SERS EF to improve the detection sensitivity. The devices in Fig. $8 \mathrm{~b}, \mathrm{~d}$ both correspond to label-free SERS biosensors with different design strategies ${ }^{174,179}$. As shown in Fig. 8b, a paper-based SERS biosensor, namely, the enPSERS biosensor, was proposed, which combines the enrichment capability for diagnosis of jaundice with sensitive and label-free detection of serum bilirubin. The enPSERS biosensor combines serum bilirubin enrichment and plasma enhancement of bilirubin Raman signals to directly improve the sensitivity of unmarked detection in serum without additional sample preparation. The key component of the enPSERS biosensor is the GO plasma $\mathrm{Au}$ nanostar hybrid densely coated on filter paper. The plasma GNS is used for SERS enhancement because of its super-high EF and built-in hot spots. In another work, a new kind of plasmon-polariton LSPR sensor, which is sensitive to reducing the size of the receptor, was described (Fig. 8d). This functionalized C-reactive protein detection system contains a single-chain antibody surface and utilizes peak shifts in the LSPR spectrum. This functional single-chain antibody is produced by the synthesis of acellular proteins, and the necessary antibodies can be quickly prepared on demand. The singlechain antibodies contain cysteine tags that bind to the surface of $\mathrm{Au}$ nanorods and, at the same time, identification sites that bind to C-reactive protein targets. The detection with the Au nanorod system using the singlechain antibody showed higher sensitivity than that with the full-length antibody as a capture probe.

\section{Potential applications}

Several mainstream potential applications of carbonbased SERS biosensors are summarized, including biomolecular detection, blood detection, bacterial detection, bioimaging, disease diagnosis and therapy, and single-cell detection and identification.

\section{Biomolecular detection (DNA, microRNA, and protein)}

Carbon-based SERS platforms have been extensively explored to develop sensitive biosensors because of their high effective surface area, excellent catalytic properties, high specificity, low $\mathrm{LOD}^{180-182}$. Xu's group ${ }^{183}$ also 


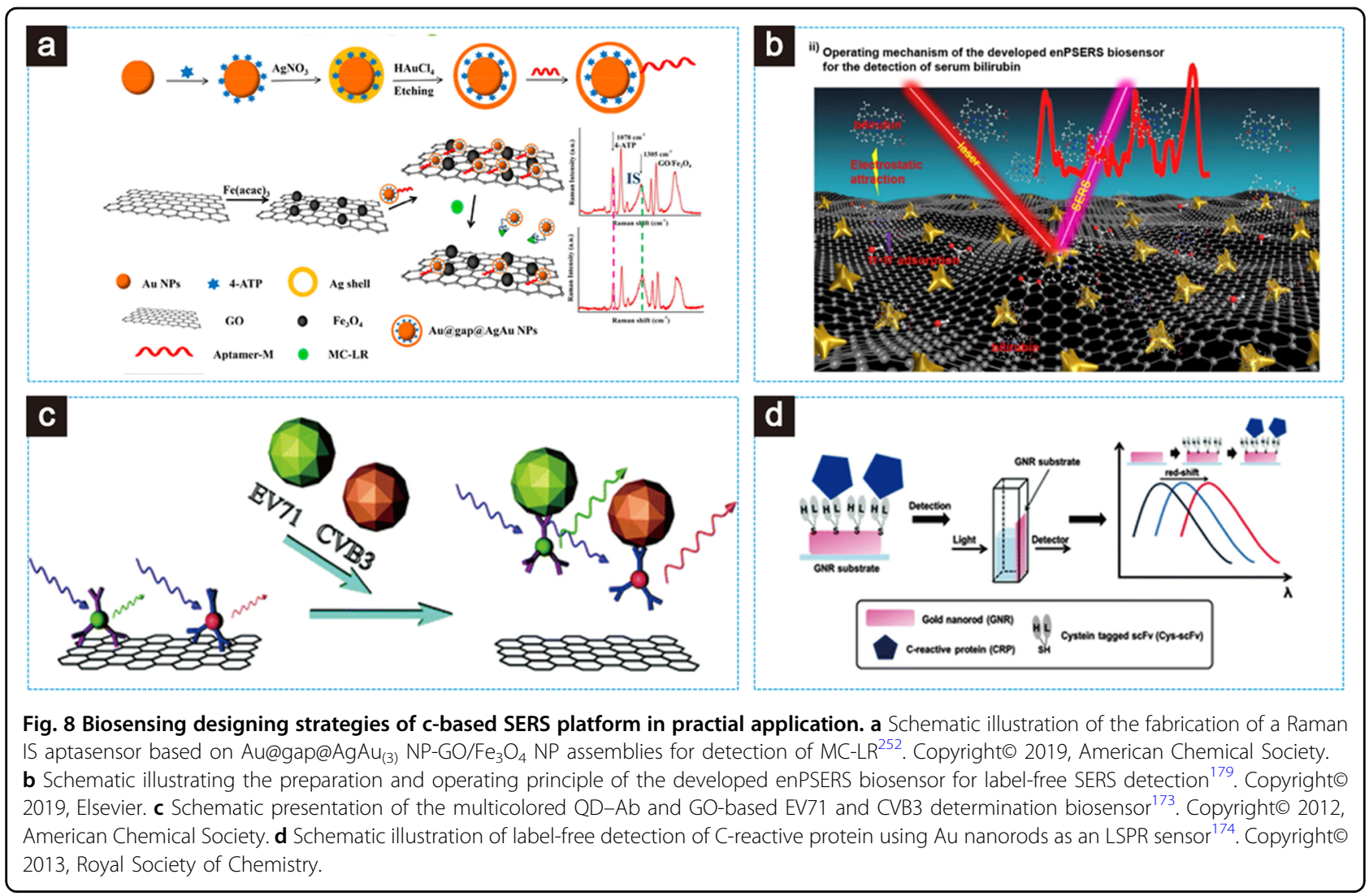

presented highly ordered arrays of graphene-isolated $\mathrm{Au}$ NPs (G/AuNPs) as a substrate to detect the concentration of adenosine, which is the core molecule of ATP and nucleic acids, with a good linear correlation within the range $2-250 \mathrm{nM}$ in human serum. In addition, $\mathrm{Xu}$ and coworkers ${ }^{184}$ developed a highly ordered graphene-isolated Ag nanodot array as a SERS substrate. This novel substrate showed excellent properties in terms of sensitivity, signal-to-noise ratio, and reproducibility because of the combination of the plasma activity of the grapheneisolated Ag nanodot array and unique physical/chemical properties of graphene, and it was employed for detecting human urinary nucleosides with a sensitivity of $91.1 \%$. Yu and co-workers ${ }^{185}$ prepared SERS-active substrates based on $\mathrm{GO} /$ silver nanocomposites via a facile and green liquid-phase reduction method. They utilized a synthetic substrate for detecting L-theanine with a LOD down to $10^{-7} \mathrm{M}$.

\section{Blood detection}

The carbon-based SERS platform plays a vital role in blood detection, such as of glucose, metallic ions, and other small molecules. In this section, we mainly present some carbon-based SERS biosensors for glucose ${ }^{186,187}$. Two-component graphene metal as a SERS substrate has been reported as a glucose detection biosensor ${ }^{188}$.
Li et al. ${ }^{189}$ utilized the SERS activity of ascorbic acid oxidized in GO and gold nanorod hybrid substrates to sensitively detect glucose. The affinity of glucose to the bare metal surface is low, and the Raman scattering crosssection, which determines the limit of glucose detected by SERS, is low. In the specific binding of glucose to the boric acid group in 4-mercaptophenylboric acid, the absolute intensity of the SERS signal of MPBA increases significantly due to the change in orientation and chargetransfer effect. However, the calculation of the peak area is complex, and the linear range of glucose concentration is limited. As depicted in Fig. 9a-d, Pham et al. ${ }^{188}$ designed a novel ternary hybrid AgNP-embedded thin silica-coated GO structure (GO@SiO $@$ @Ag NPs) to detect glucose within the linear range 2-20 mM (Fig. 9d), which was achieved by self-assembling 4-mercaptophenyl boronic acid on the surface of $\mathrm{GO} @ \mathrm{SiO}_{2} @ \mathrm{Ag} \quad \mathrm{NPs}$ as GO@SiO ${ }_{2} @ A g N P s @ M P B A$ (Fig. 9a, c). The binding of glucose to 4-mercaptophenyl boronic acid-incorporated GO@SiO ${ }_{2} @ \mathrm{Ag}$ NPs increased the SERS signals at both 1072 and $1588 \mathrm{~cm}^{-1}$ (Fig. 9b). In addition, Guo et al. ${ }^{190}$ also developed ternary $\mathrm{Ag}-\mathrm{Cu}_{2} \mathrm{O} / \mathrm{rGO}$ nanocomposites as SERS biosensors by a facile two-step in situ reduction procedure at room temperature to detect $\mathrm{H}_{2} \mathrm{O}_{2}$ and glucose (Fig. 9e-h). Glucose detection was demonstrated with a high sensitivity and could be employed to 
a

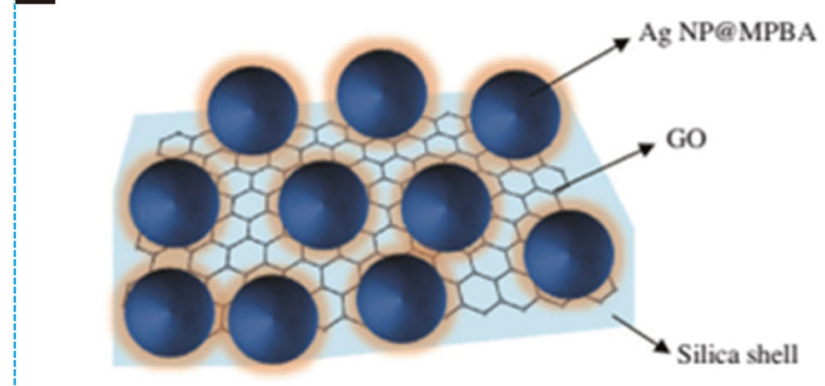

C

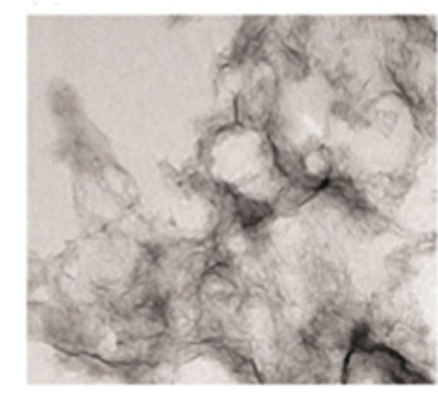

b

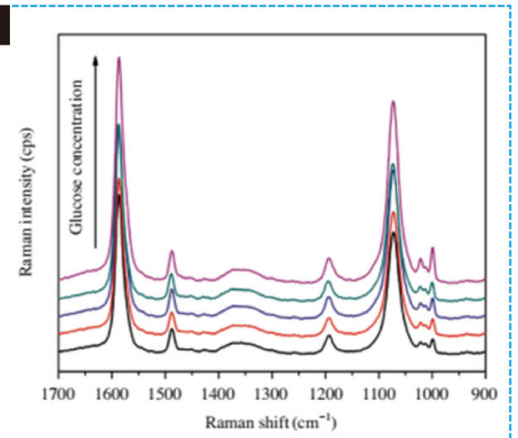

d
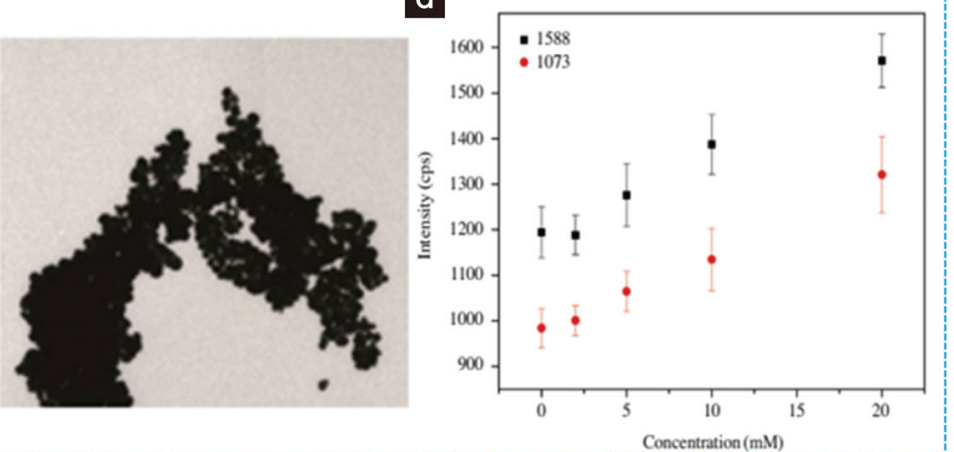
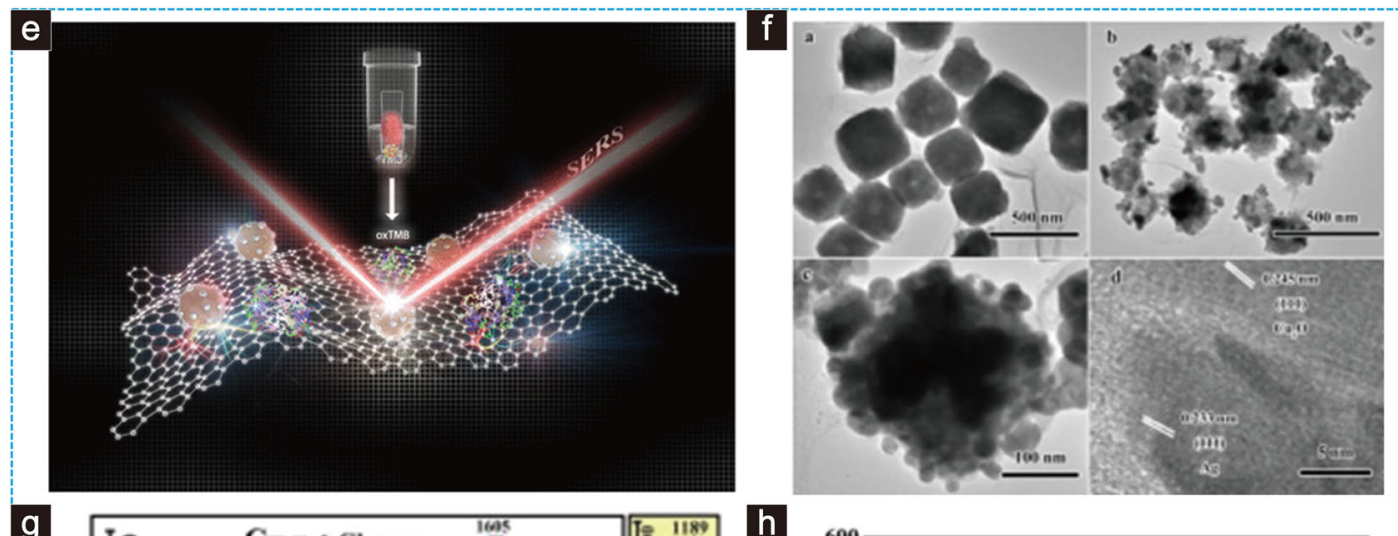

g
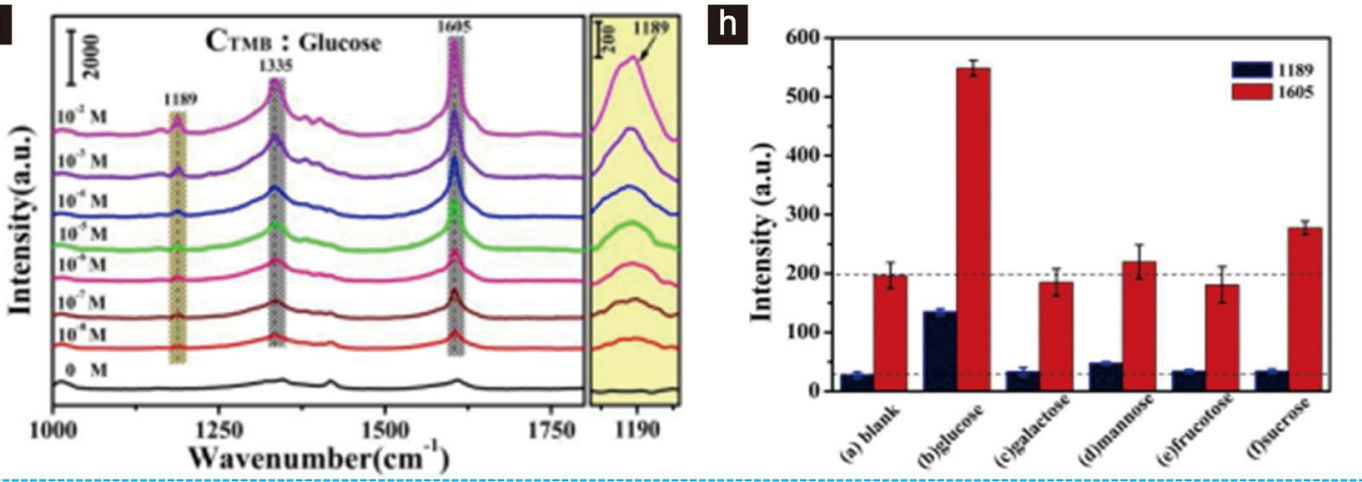

Fig. 9 Carbon-based SERS platform for blood detection. a Structure diagram of silver nanoparticle-embedded silica-coated graphene oxide

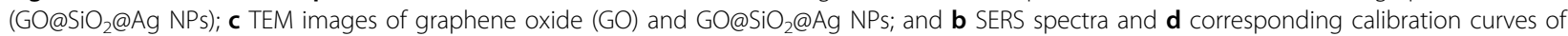
GO@SiO $@$ @AgNPs according to glucose concentration in PBS buffer ( $\mathrm{pH} 3.0)^{188}$. Copyright@ 2016, Springer. e Schematic illustration of synthetic procedure for $\mathrm{Ag}-\mathrm{Cu}_{2} \mathrm{O} / \mathrm{rGO}$ nanocomposites as SERS biosensors; $\mathbf{f}$ TEM and HRTEM images of the prepared $\mathrm{Cu}_{2} \mathrm{O} / \mathrm{rGO}$ and $\mathrm{Ag}-\mathrm{Cu}_{2} \mathrm{O} / \mathrm{rGO}$ nanocomposites; $\mathbf{g}$ SERS spectra of oxidized TMB molecules on the surface of $\mathrm{Ag}-\mathrm{Cu}_{2} \mathrm{O} / \mathrm{rGO}$ substrates in the presence of different glucose concentrations; and $\mathbf{h}$ high selectivity of $\mathrm{Ag}-\mathrm{Cu}_{2} \mathrm{O} / \mathrm{rGO}$ SERS biosensor in glucose detection ${ }^{190}$. Copyright $\odot 2017$, American Chemical Society. 
distinguish between diabetic and normal individuals by using the glucose levels in fingerprints (Fig. 9g-h). Ag$\mathrm{Cu}_{2} \mathrm{O} / \mathrm{rGO}$ nanocomposites have been proven to be a highly efficient SERS substrate and highly sensitive peroxidase nanocatalysts. The most important contribution of this work is that this method can distinguish the blood sugar levels of diabetic patients and normal people from fingerprint information and can be used for in situ detection of glucose levels in wearable devices for diabetic patients. In the near future, the strategies developed by this work will show promise for disease diagnosis and forensic investigation.

\section{Bacteria detection}

SERS can be used to identify specific frequencies of chemical functions in complex aquatic biological samples via convenient visible and NIR laser probes. Based on metal colloids, CNT templates, and selfassembled membranes, SERS biosensor technology has been developed to improve the sensitivity and accuracy. However, most of the approaches still face the challenges of low reproducibility and low stability. SWCNTs have the characteristics of a radial breathing mode and tangential Raman scattering. As a support for AuNPs, they may become a practical hybrid SERS substrate. These structures produce sharp and intense peaks that are easily distinguished from the fluorescent background. To improve the stability and photothermal properties, f3-SWCNTs were chosen as a template to prepare $\mathrm{Au}$ nanopopcorn (Fig. 10a). Selectivity is integrated by targeting specific recognition of monoclonal antibodies. A novel hybrid nanomaterial based on gold nanopopcorn-attached SWCNTs (AuNP@f3SWCNTs) was fabricated successfully to realize labelfree detection and photothermal killing of bacteria by Hamme II's group (Fig. 10a, b) ${ }^{191}$. The application potential of SWCNT/AuNP nanohybrid materials is enhanced by the large aspect ratio and good combination with biological molecules. A current challenge is to detect and efficiently eliminate antibiotic-resistant bacterial strains. Specifically, the outer membrane of Gram-negative bacteria provides additional protective layers, such as against antibiotics and fungicides, which help bacteria resist antibiotics. To address this challenge, Ko et al. ${ }^{192}$ fabricated an $\mathrm{Ag} / \mathrm{ZnO} / \mathrm{rGO}$ nanocomposite as a multifunctional biomedical platform for SERS detection and multiple killing of E. coli. Importantly, this work was the first report of combining three materials for simultaneous detection and killing of bacteria. Recently, a simple and environmentally friendly synthetic method for preparing an effective SERS platform has attracted much attention: Li et al. ${ }^{193}$ proposed a fast and green method to prepare AgNP/ rGO composites (Fig. 10c) and utilized the fabricated
SERS substrate to detect bacteria such as $E$. coli and $S$. aureus with a detection sensitivity as low as $1 \times 10^{5}$ $\mathrm{CFU} / \mathrm{cm}^{3}$. The simple and environmentally friendly in situ synthesis method has potential application prospects in the preparation of other graphene/metal matrix nanocomposites.

\section{Bioimaging}

Fluorescence technology has been widely used in biological imaging; however, there still exist some problems, such as photobleaching of organic dyes, autofluorescence background of biological tissue, and wide fluorescence excitation and emission peaks, which lead to spectral overlap and limit the usage of a variety of colors in practice. Raman scattering has narrow spectral lines and can be used for high multiplexing imaging ${ }^{194}$. In addition, because the sharp Raman peaks can be distinguished from the fluorescence background, the autofluorescence problem of the tissue can be bypassed. Raman excitation can also be selected in a low background and biologically transparent optical window. Recently, SERS has emerged as a fascinating and new powerful tool for bioimaging ${ }^{195}$, mainly in applications of cell tracking, cell targeting, diagnosis therapy, and so on ${ }^{169,196,197}$. Raman imaging technology has broad application prospects in the field of next-generation biological imaging.

The two most commonly used types of Raman biological imaging technology mainly include SERS imaging technology based on material autofluorescence and FSERS imaging technology, as discussed before. Compared with small-molecule Raman probes such as $p$-aminothiophenol and R6G, carbon-based nanomaterials are less toxic to living cells, and more importantly, carbon nanomaterials can be designed as a platform to load and carry molecules with different functions, including drugs and targeted species ${ }^{54-56}$. Therefore, carbon nanomaterials have great potential as a multifunctional and feasible biomedical imaging platform for diagnosis and treatment with the two technologies. First, for carbon nanomaterial autofluorescence bioimaging, the signal enhanced by SERS technology makes it possible to carry out fast Raman detection and spatial mapping of the target with lower laser power and shorter acquisition time in actual imaging. In addition, thermal effects and subsequent damage to cells or tissues can be minimized. Therefore, depending on the SERS technology, GO, as an effective CM-enhanced substrate, can be used as a promising probe for in vivo Raman imaging. For example, based on the cell uptake mechanism of a GO-based SERS nanomaterial and its good biocompatibility and water solubility, the enhanced $1595 \mathrm{~cm}^{-1}$ characteristic peak of the Gband was used to directly detect GO in living cells ${ }^{198}$. The G-band integral intensity was used to map the cells to obtain Raman images, which can provide subcellular 


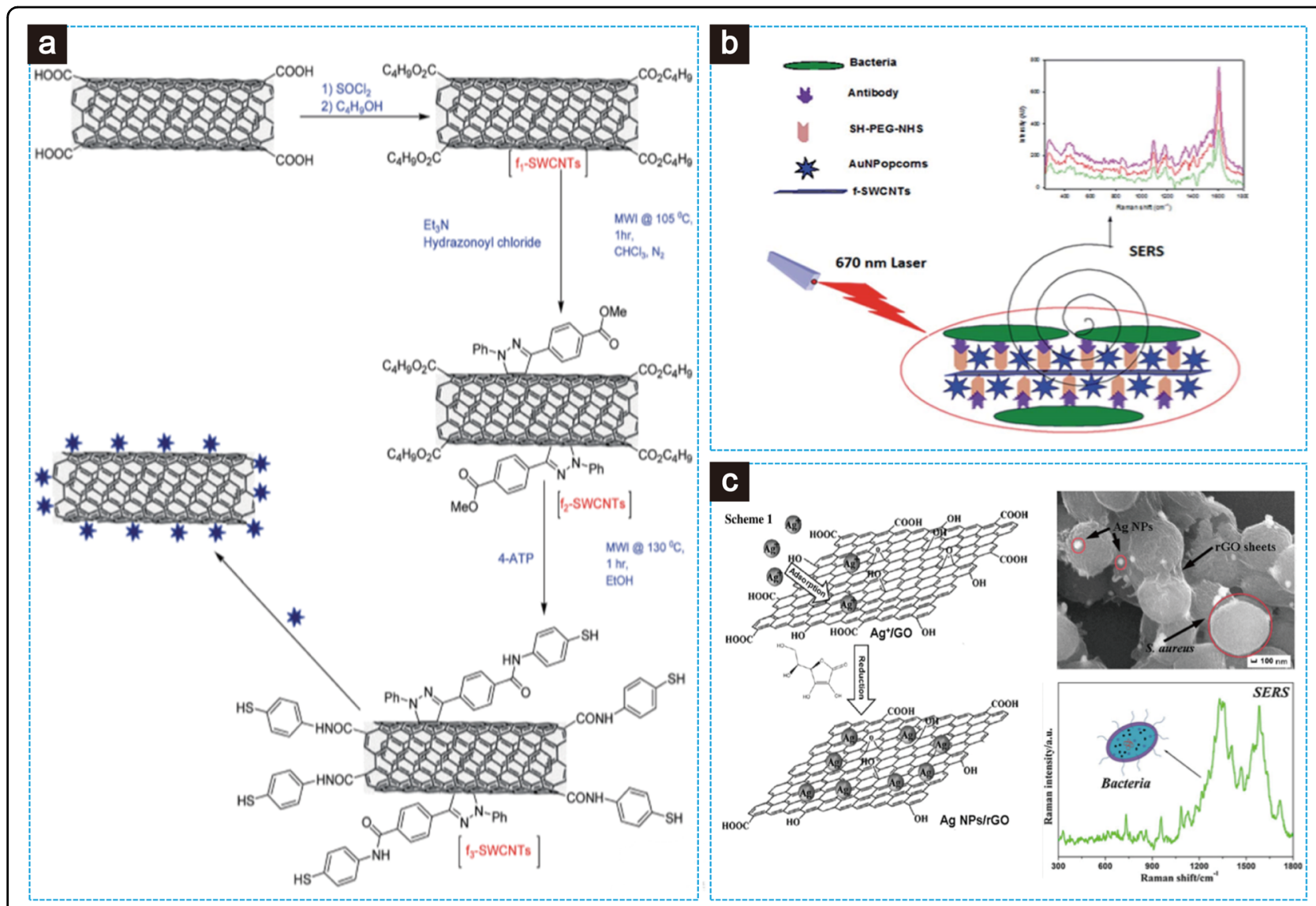

Fig. 10 Carbon-based SERS platform for bacteria detection. a Microwave-promoted 1,3-dipolar cycloaddition and amination of ester-modified SWCNTs and attachment of gold nanopopcorns; $\mathbf{b}$ scheme of the synthesis of the antibody-conjugated AuNP@f3-SWCNT SERS probe hybrid nanomaterial and detection of bacteria ${ }^{191}$. Copyright@ 2014, Springer. c Synthesis process scheme for a AgNP/rGO composite as a SERS biosensor, SEM image of the mixture containing the AgNP/rGO composite and S. aureus, and schematic of bacteria detection by SERS ${ }^{193}$. Copyright $\odot 2017$, Springer Nature.

localization sensing under a short acquisition time and a low laser power. In addition, as one-dimensional quantum wires, SWCNTs have a strong density of electronic states at Van Hove singularities and intrinsic optical properties, including NIR photoluminescence and strong resonance Raman scattering, both of which have been used in biological imaging. Monochromatic SWNT Raman imaging and single-walled carbon polychromatic Raman bioimaging of biological samples have been reported ${ }^{194,199}$. However, autofluorescence SERS bioimaging has shortcomings such as reduced time efficiency, high background staining, and antigen transformation by solvents. The multiplex F-SERS dot approach using a topical spray can detect tumor biomarkers in the bona fide tissue environment without any endoscopic manipulation or without major skin removal in the case of intraoperative imaging. Carbon-based F-SERS technology enables a huge encoding capacity for high-throughput biodetection by using the joint F-SERS signal as the encoding element. Due to the use of photoluminescence and SERS spectral regions, the spectral range that can be used for encoding is broadened, thus allowing more spectrally distinguishable codes $^{54,200}$. In addition, when the amount of code available increases, the encoding capacity can be further increased. In addition, based on the ability to rapidly image fluorescence signals and simultaneously detect SERS signals, optical endoscopic imaging systems for specific tumors can be successfully realized by using optical fiber bundles for real-time, in vivo, and multitarget detection in intraoperative endoscopic systems ${ }^{56,176,201}$, and the details will be explained in the following discussion.

A GIAN, as a graphite nanomaterial, has a large Raman scattering cross-section $\left(\sim 10^{-21} \mathrm{~cm}^{2} \mathrm{SR}^{-1}\right.$ molecule $\left.{ }^{-1}\right)$ and is stable based on its inorganic structure. Two distinct bands (Fig. 11a) can be observed near 1325 and $1595 \mathrm{~cm}^{-1}$, corresponding to the D and G Raman modes of graphite carbon shells, respectively. The high strength 
(relative to the G peak) Raman D peak reflects the high strain of the graphite shell caused by the deformation of flat graphene encapsulated with Au nanocrystals. A GIAN has strong and simple resonance Raman characteristics and can be used as a good Raman marker for cell imaging. Figure 11a-c shows Raman images of MCF-7 breast cancer cells stained with GIAN. Both the D-mode and Gmode can be used for cell imaging. The Au nanocrystalline nuclei significantly enhanced the Raman signal of the graphene shell and made MCF-7 cells luminescence under laser excitation. All Raman signals are distributed in the cytoplasm, but no signal is related to the nucleus, indicating the GIAN distribution in the cell. Compared with fluorescence imaging, the Raman signal of MCF-7 cells has good imaging resolution, which shows that the half-peak width of the Raman scattering peak is small and the unbleached GIAN Raman signal is stable. This is a potentially useful tool for monitoring the real-time process of GIAN in cells. As depicted in Fig. 11d, Chen et al. $^{202}$ proposed an excellent new theragnostic nanosystem in which rGO nanosheets are considered a platform to construct porous silica shells on the surface of rGO with encapsulated R6G and AuNPs (anti-EGFRPEG-rGO@CPSS-Au-R6G). It is worth mentioning that anti-epidermal growth factor receptor (EGFR) is a kind of antibody against target cells. A SERS mapping image was utilized to observe the targeting specificity of the presented substrate and others (Fig. 11e). It can be seen that Raman signals are mainly distributed in the cytoplasm, not in the nucleus. Compared with fluorescence imaging, the Raman signal bandwidth of MCF-7 is narrower and has a higher resolution. In addition, the fluorescent molecules easily self-quench, and the Raman signal is more stable. The development of multimodal systems using nanomaterials has yielded cutting-edge biomedical functions.

Compared with CNTs and graphene, GO has good water solubility and biocompatibility. Thus, GO can be used as a flexible Raman probe to image cells or tissues by Raman mapping. In addition, thermal effects and subsequent damage to cells or tissues can be minimized. Therefore, based on SERS, GO is expected to become a promising Raman imaging probe in vivo. As shown in Fig. $12 \mathrm{a}, \mathrm{b}$, a simple and highly repeatable procedure using GO as a Raman probe based on the SERS imaging effect was proposed. GO modified by AuNPs is internalized into a single cell, providing a local sensing level in the subcell. Because of the SERS effect, the sensitivity is greatly improved, and the capture time is effectively shortened. These results indicate that the in situ modification of AuNPs on GO nanosheets successfully triggers the SERS effect, thus enhancing the Raman intensity of cells and ultimately improving the Raman imaging of cells. Combining AuNPs with CNTs is one good way to overcome the relatively long spectral acquisition times. An SWNT has many unique optical properties. It is a new nanoprobe widely explored in biomedical imaging and phototherapy. Thus, Wang et al. ${ }^{203}$ designed hybrid nanocomposites composed of AuNP/SWNT conjugates coated with PEG for in vitro Raman imaging (Fig. 12c, d). DNAfunctionalized SWNTs were modified by noble metal (Ag or $\mathrm{Au}$ ) NPs via an in situ phase synthesis method. The method includes seed attachment, seed growth, and surface modification with PEG, yielding SWNT-Ag-PEG and SWNT-Au-PEG nanocomposites that are stable under physiological environments (Fig. 12d). Using a NIR laser as the excitation source, targeted Raman imaging of cancer cells labeled with the folic acid (FA)-conjugated SWNT-Au nanocomposite (SWNT-Au-PEG-FA) was realized, and cancer imaging and therapy could thereby be achieved (Fig. 12c). Compared with the nonenhanced SWNT Raman probe, the image was obtained in a significantly shorter time. Because of the strong surface plasmon resonance absorption of the Au shell, SWNTAu-PEG-FA nanocomposites also have an obvious photothermal killing effect on cancer cells.

\section{Disease diagnosis and therapy}

Carbon nanomaterials, such as CNTs and graphene, selectively accumulate in tumors according to electron paramagnetic resonance and exhibit extremely high intrinsic absorption in biotransparent windows located in the NIR region $(750-1700 \mathrm{~nm})^{204,205}$. Graphene has NIR absorption characteristics and is suitable for photothermal therapy. The strong Raman signal makes it an important Raman marker for bioimaging. SWCNTs were the first carbon nanomaterials used for photothermal treatment ${ }^{89,206,207}$. In addition, the strong fluorescence emission wavelength of carbon nanomaterials ranges from visible light to the NIR-IR window, allowing simultaneous imaging and treatment. Compared with other nanomaterials (including $\mathrm{Au}$ nanorods and nanospheres for photothermal therapy), the use of carbon nanomaterials is advantageous. First, through a proper surface modification to prevent immune system recognition, CNTs and graphene can circulate in vivo with half-lives approaching several days, which is a significant improvement. The possibility that intravenously injected carbon nanomaterials are trapped in the tumors leads to the super-high tumor absorption levels. Second, CNTs and graphene offer additional benefits because they are ideal for strong light absorption in the NIR region, which has better tissue penetration than light in the visible region ${ }^{208,209}$.

Materials for photothermal therapy ${ }^{210}$ should be considered based on their high NIR absorption so that the SERS substrate can absorb in the NIR spectrum to increase the temperature of the surroundings and cause target cell death ${ }^{122,211,212}$. SERS has shown great potential 

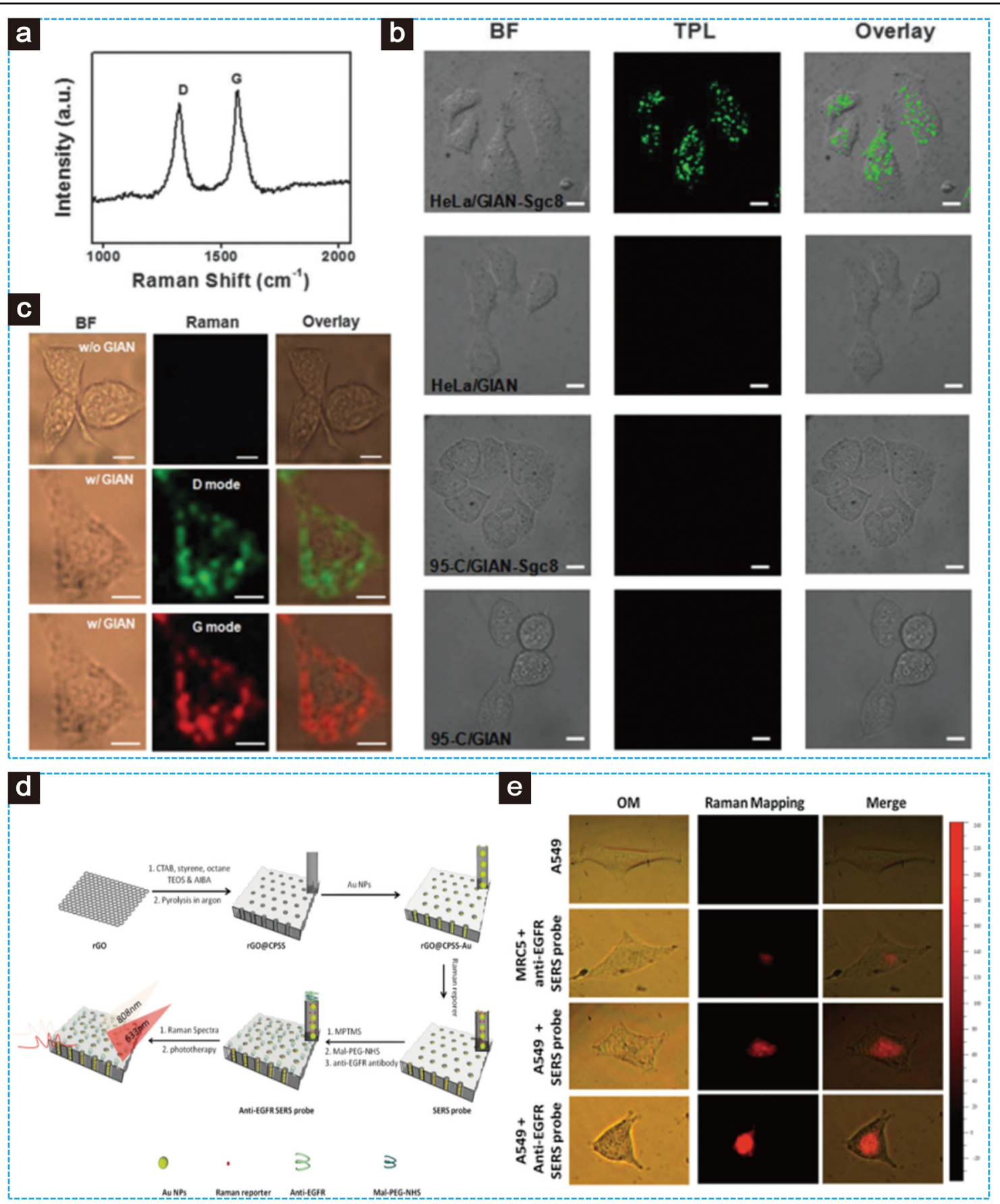

Fig. 11 Multimodal cell imaging with GIAN. Multimodal cell imaging with GIAN: a Raman spectrum of GIAN (633 nm excitation). b Bright-field images of MCF-7 cells, and Raman images obtained by monitoring the D- and G-mode shown in (a). c Bright-field images of HeLa cells and 95-C cells incubated with GIAN and GIAN-Sgc8, and two-photon luminescence images demonstrating cell selectivity ${ }^{132}$. Copyright $\odot 2014$ Nature Publishing Group. $\mathbf{d}$ Fabrication of a SERS probe, and e optical image, Raman mapping image and merged image of A549 cells as a control, MRC-5 cells with the anti-EGFR-SERS probe, A549 cells with the SERS probe, and A549 cells with the anti-EGFR-SERS probe ${ }^{202}$. Copyright@ 2016, Wiley.

in photothermal cancer therapy and photothermally enhanced chemotherapy ${ }^{196}$. Tan and co-workers ${ }^{133}$ developed a simple CVD method to fabricate GIAN nanostructures, which encapsulate $\mathrm{Au}$ nanocrystals within a thin layer of graphene and were used for bioimaging and cancer therapy ${ }^{132}$. Figure 13 shows the photothermal-enhanced chemotherapy progress with GIAN. A thin layer of graphene is accurately deposited on 


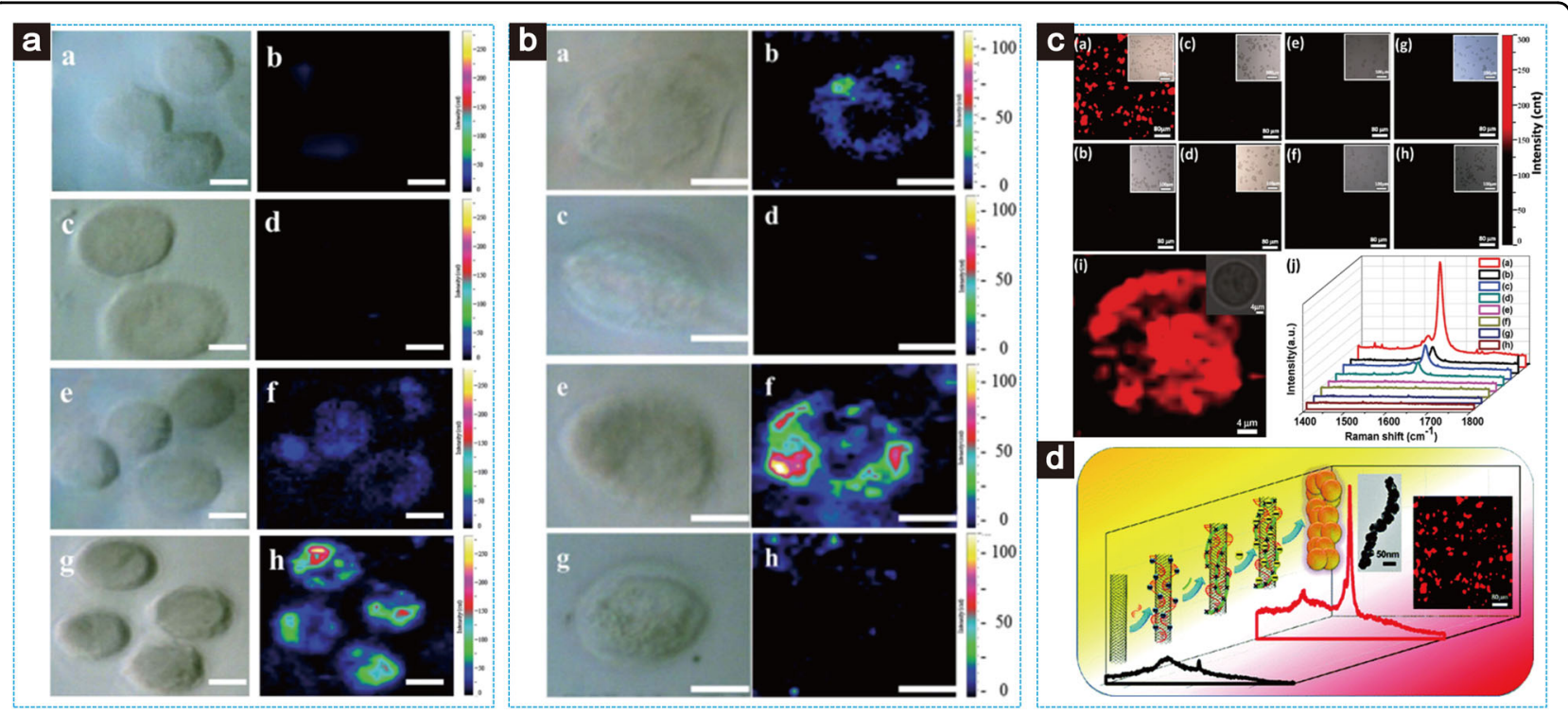

Fig. 12 Typical GO and SWNT-based SERS platform for bioimaging. a Investigation of the cellular uptake mechanism of GO and Au/GO hybrids. The left panels are optical images, while the right panels are the corresponding Raman images (scale bar: $5 \mathrm{~mm}$ ). b Optical (a, c, e, and g) and Raman ( $b, d, f$, and h) images of HeLa 229 cells $^{198}$. Copyright@ 2012, Royal Society of Chemistry. c Raman images of SWNT-Au-PEG-FA-, SWNT-Au-PEG-, SWNT-PEG-FA-, and SWNT-PEG-labeled KB and HeLa cells, where the nanotube concentrations in all samples were kept constant at 5nM. Highresolution Raman image of an SWNT-Au-PEG-FA-labeled KB cell. The insets in parts (a-i) correspond to bright-field images of cells; the averaged Raman image is shown in each image above. $\mathbf{d}$ Schematic illustration of the synthetic procedure of SWNT-Au-PEG-FA nanocomposites as SERS biosensors for bioimaging ${ }^{203}$. Copyright@ 2012, American Chemical Society.

the surface of $\mathrm{Au}$ nanocrystals, which has unique properties. First, as a SERS substrate, GIAN quench the background fluorescence and reduce the photocarbonization or photobleaching of the analyte. Second, GIAN can be used for multimode imaging of cells based on Raman scattering and NIR two-photon emission (Fig. 13b). Third, GIAN provide a platform for treatment with anticancer drugs such as DOX (doxorubicin) (Fig. 13a). Finally, their NIR absorption properties were combined for GIAN photothermal therapy and chemotherapy. As depicted in Fig. 13c, HeLa cells could be imaged by GIAN TPL, whereas 95-C cells remained dark. It is believed that the SPR of the Au core and two-photon absorption by the graphene shell both contribute to the TPL signal, as bare AuNPs and hollow carbon capsules both exhibit the TPL effect. The controlled release of DOX in GIAN is achieved by NIR heating, which significantly reduces the possibility of side effects of chemotherapy. GIAN have a high specific surface area, a stable thin shell structure and unique optical and photothermal properties, which enable them to have broad applications in biomedicine. These GIAN show good stability in harsh environments. The thin graphene shell shows a specific Raman character and can be optimized for cell staining and imaging. Designing aptamer-functionalized GIAN via strong $\Pi-\Pi$ interactions can realize targeted cell imaging. The stained GIAN with cancer cells under irradiation by an $808 \mathrm{~nm}$ laser can kill the cancer cells effectively, suggesting that this SERS substrate can be used for NIR photothermal therapies.

\section{Single-cell detection and identification}

Intracellular or extracellular single-cell analysis plays an important role in elucidating cell diversity and heterogeneity and helps reveal the related pathways of cell heterogeneity and disease status, which are concealed by a large number of measurements. Compared with traditional culture-based detection methods, integrated nanotechnology biosensors have obvious advantages, such as high-throughput, low detection limit, reduced sample size and the possibility of being label-free, and are expected to meet the needs of actual pathogen diagnosis analysis. In addition, a plasma biosensor can be used conveniently and quickly (within 5-15 min) ${ }^{213-216}$. Single-cell detection and identification can be realized by relying on SERS due to the fingerprint spectrogram, which combines easy production at low cost with a high enhancement enabling single-cell detection ${ }^{217-220}$. The most common urinary pathogens are detected by an unlabeled SERS-based detection platform, and strain levels are distinguished by using microarray immobilization coupled with in situ AgNP synthesis and principal component analysis data to identify individual bacterial cells. To use SERS for singlecell detection steadily and reliably in practical applications, it is necessary to control the stability of 

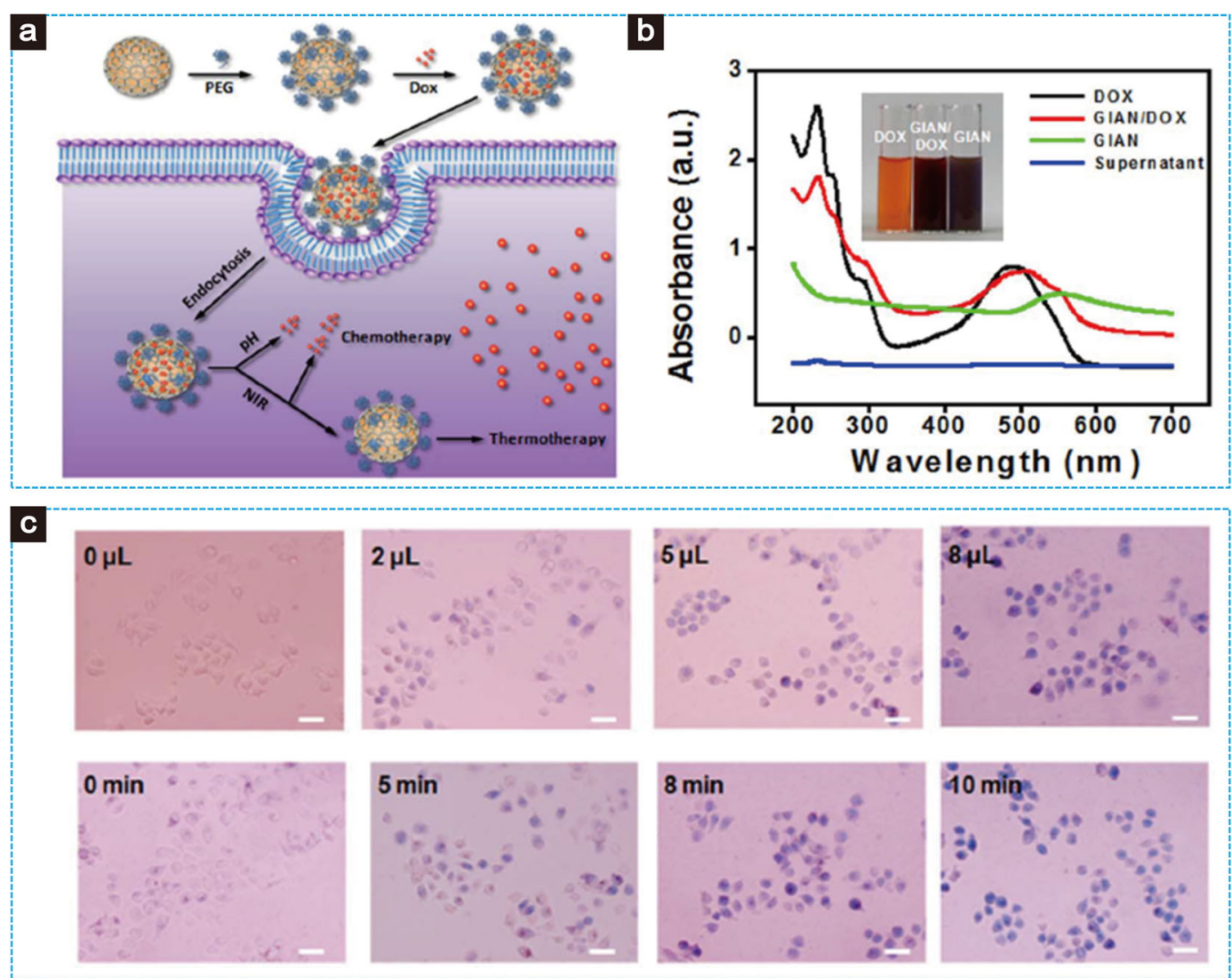

Fig. 13 Photothermal-enhanced chemotherapy progress with GIAN. Photothermal-enhanced chemotherapy progress with GIAN. a Schematic of the mechanism of NIR photothermal-enhanced chemotherapy progress with GIAN; $\mathbf{b}$ UV-Vis spectrum of DOX-loaded GIAN; and $\mathbf{c}$ bright-field microscopy images of Trypan blue-stained MCF-7 cells after different NIR photothermal treatments. Scale bar: $50 \mathrm{~mm}^{132}$. Copyright@ 2014 , Nature Publishing Group.

nanostructures and plasma effects around hot spots, thus limiting the fluctuation of SERS signals, which hinders the repeatability ${ }^{221,222}$. This again demands a better understanding of the origins of the highly enhanced single-cell signal. Dina et al. $^{223}$ provided a label-free SERS-based biosensor for Gram-negative and Gram-positive microorganism detection that reached the single-cell level. This biosensor based on AgNPs displayed a rapid (within $5 \mathrm{~min}$ ) and label-free character without the use of antibodies or other specific receptors.

As shown in Fig. 14a-c, a unique SERS-based nonplasma biosensor platform was introduced that uses biocompatible self-assembled 3D interconnected carbon nanonetworks to detect and differentiate HeLa cells and fibroblasts in vitro. The schematic diagram in Fig. 14a illustrates the composition of a 3D interconnected carbon nanonetwork platform. In an atmospheric environment, the surface of the graphite sheet is treated by a femtosecond laser, and photon energy-induced ionization is introduced into the graphite sheet. A self-functional 3D interconnected carbon nanonetwork structure is synthesized. Under the precise guidance of the programmable galvanometer scanner, the predetermined line array is transferred to the graphite surface when the laser beam of the scanner can move in two dimensions. The surface of the graphite sample is ablated, and a vapor plume containing NPs is formed by the laser treatment. The charge-transfer mechanism principle and the 3D interconnected nanocarbon web cluster uptake process by both fibroblasts and HeLa cells during the incubation period are shown in Fig. 14b. Cell uptake provides the necessary local light field for ultrasensitive intracellular detection. Figure 14c shows that both fibroblasts and HeLa cells are present during incubation. The fluorescence microscopy result shows that interconnected carbon nanonetwork clusters are in the fibroblasts and HeLa cells. This biosensitive platform attracts cells as a region with high protein adsorption, and primary attachment occurs.

Although several SERS sensors have been developed to locally detect intracellular species levels, this technique is still in the early stage of characterizing individual 

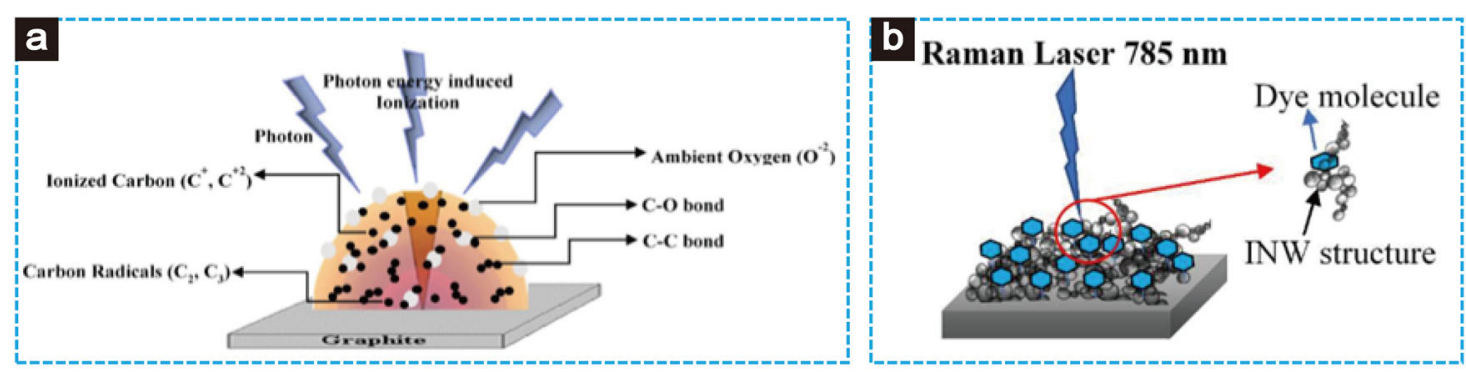

c
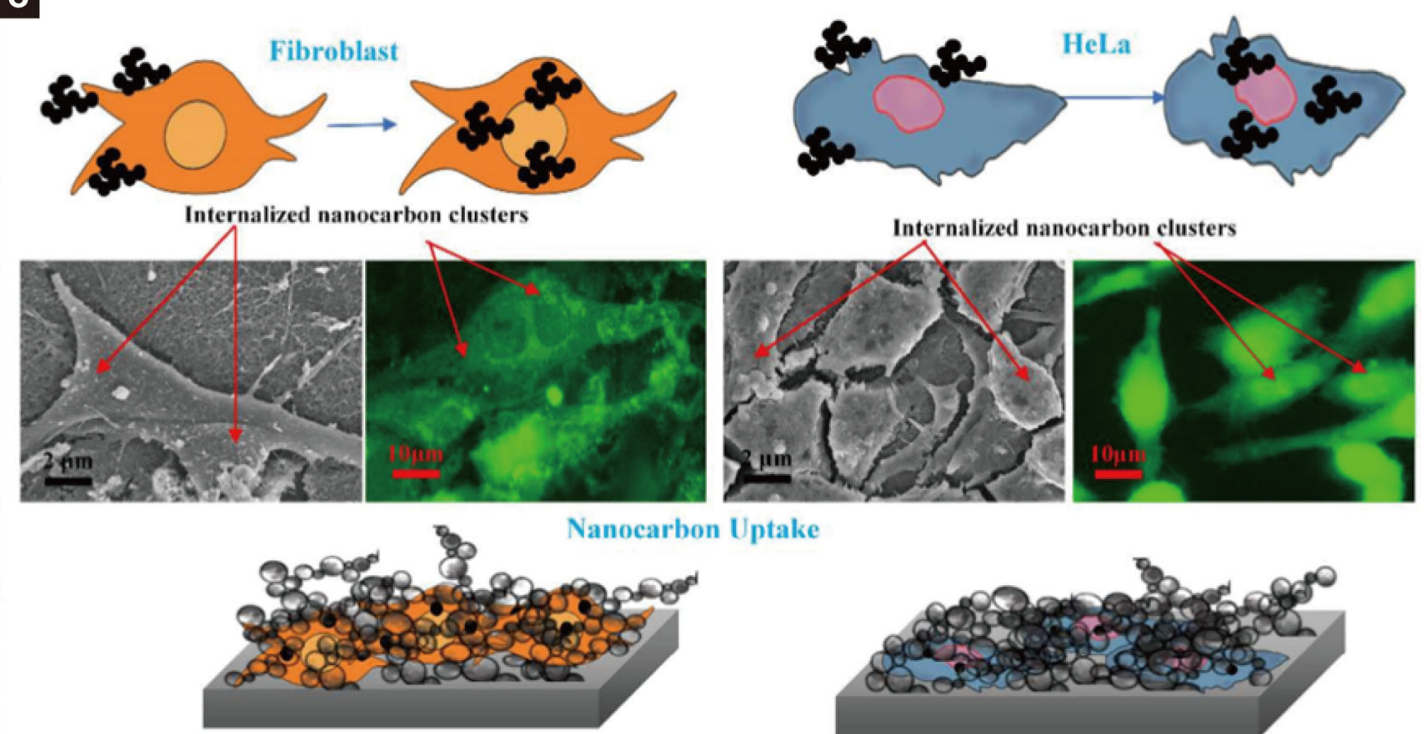

Fibroblast

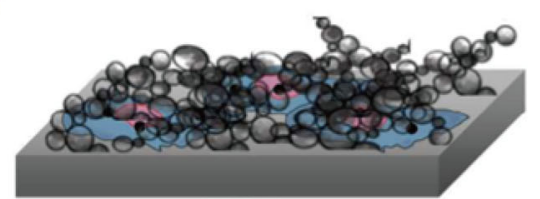

HeLa

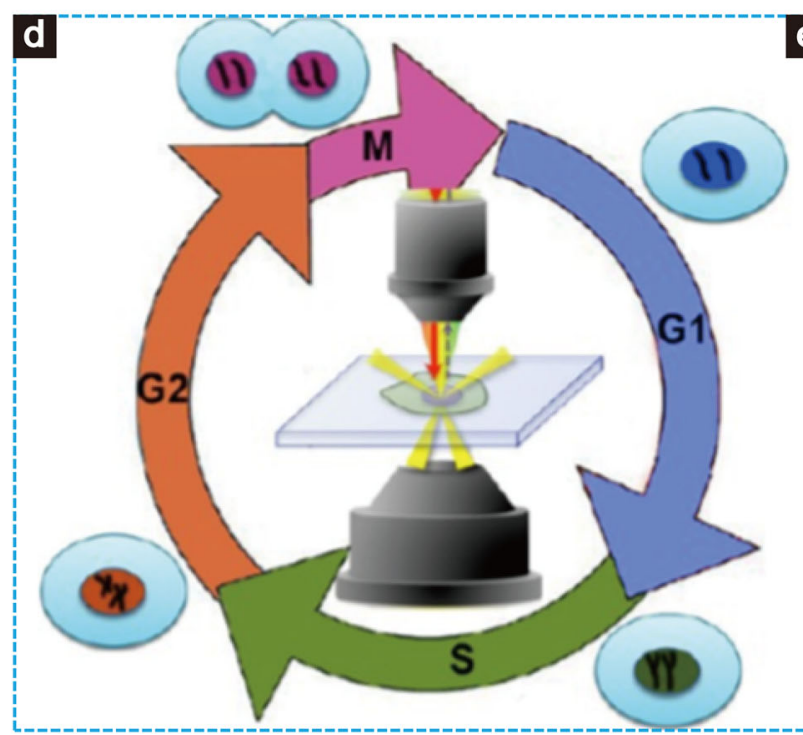

$\Theta$

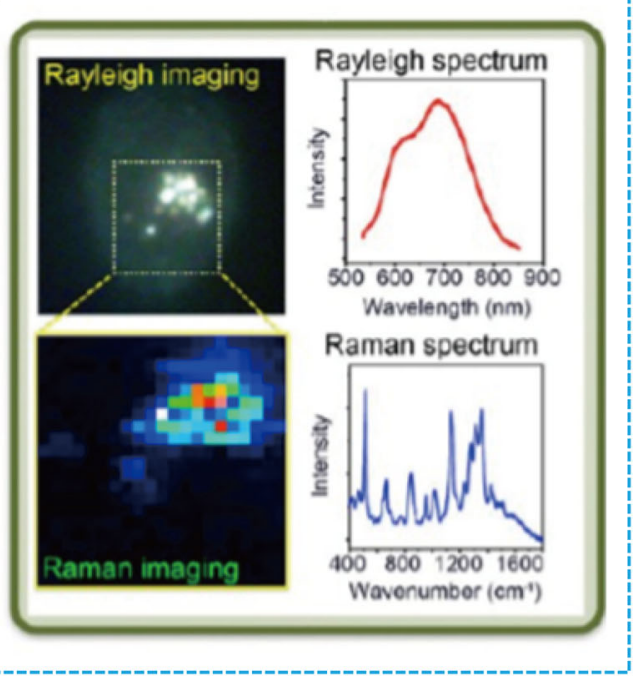

Fig. 14 Typical carbon-based SERS biosensors for single-cell detection and identification. a-c SERS-active 3D interconnected nanocarbon web for nonplasma in vitro sensing of HeLa cells and fibroblasts: a single-stage 3D interconnected nanocarbon web platform synthesis illustrated schematically; b charge-transfer mechanism principle; and c 3D interconnected nanocarbon web cluster uptake process by both fibroblasts and HeLa cells during the period of incubation ${ }^{253}$. Copyright@ 2018, American Chemical Society. d Experimental design of targeted plasma-enhanced singlecell Rayleigh/Raman spectroscopy to monitor the molecular changes of any cell component, and e corresponding Raman images results from a single living cell ${ }^{232}$. Copyright@ 2012, American Chemical Society. 
extracellular metabolites ${ }^{224-227}$. The application of microwells, micromodes, microfluidic devices, and traps in cell limitation and operation has achieved varying degrees of success. Endogenous hot spot factors are an effective way to improve SERS sensors by enhancing signals in restricted single cells ${ }^{228-231}$. Nolan et al. ${ }^{224}$ pioneered SERS flow cytometry to analyze SERS markers attached to micron-sized beads or individual cells. Kang et $\mathrm{al}^{232}$ proposed a new technique, named targeted plasmonically enhanced single-cell Raman/Rayleigh spectroscopy. This technique can perform cell imaging and molecular monitoring of target cell components (i.e., nucleus, cytoplasm, cell membrane, etc.) throughout the cell cycle (Fig. 14d, e). A labeled graphene/AuNP SERS platform, combined with multivariate analysis and principal component analysis, was used to describe live, dead, and burst colon cancer cells suspended in simulated body fluids (Fig. 14d). Graphene sheets allow SERS hot spot recognition and provide chemical enhancement for biological components. The study found unique fingerprints of three different states of cells: burst, live, and dead, which were used to distinguish p53+/+ from p53-/ - cell lines and to achieve the possibility of single gene differentiation $^{233}$. Current noninvasive platforms usually rely on the detection of cells or proteins from biological fluids such as blood, which provides insufficient information on the progression and metastasis of tumors. Therefore, simultaneous detection of cancer cells and proteins can further advance current cancer detection strategies. Reza et al. $^{234}$ used GO functionalization to increase the active surface area and, more importantly, to reduce the functional

steps for rapid detection. Alternating-current electrohydrodynamic fluid flow provides fine mixing to enhance target-sensor interactions, thereby minimizing nonspecific binding and Se for multiple detections. They detected cancer cells and monitored the heterogeneous expression of cell surface proteins, which may be helpful for the clinical identification of effective patient treatment. Heterogeneous expression of human EGFR 2 protein on the surface of a single cancer cell was reported. This ability has great potential in clinical early cancer diagnosis and treatment monitoring.

\section{Conclusions and perspectives}

The recent developments described in this review show that carbon-based SERS biosensors are being increasingly applied in biological science. SERS is a highly sensitive technique that allows for the detection of molecules at very low concentrations and provides rich structural information in the detection of various biological samples and diseases, including DNA, microRNA, protein, whole blood, and bacteria; bioimaging; disease diagnosis or therapy; and single-cell detection and identification.
Nevertheless, there are still some opportunities and challenges. For example, to synthesize carbon-based SERS substrates with higher sensitivity and reproducibility, researchers must be able to prepare high-performance single NP-based SERS substrates, precisely control the location of hot spots between particles, and generate ultra-small labels for single molecules. Based on the excellent electrical properties of carbon nanomaterials, carbon-based SERS substrates combined with semiconductor chips and microelectronic processing technology will realize further developments in the flexible microelectronic field and potentially be employed as multifunctional biosensors with in situ analysis and quantitative analysis abilities in vivo or other real applications. There is no doubt that graphene plays an important role in SERS, and it is expected to enable a breakthrough in future research to meet practical applications, although this cannot be completed in one action. From an application perspective, the relationship between the sensitivity, selectivity, and repeatability needs to be carefully prioritized. With the deepening of environmental problems and economic considerations in recent years, green analytical chemistry has become a key area of analytical chemistry, simplifying sample preparation and measurement procedures to realize the dream of rapid analysis and in situ real-time monitoring with carbonbased SERS biosensors. In-depth study of carbon-related Raman enhancement will help us understand not only promising SERS substrates but also the CM.

Looking into the future, as depicted in Fig. 15, there is a need to develop carbon-based SERS biosensors that allow not only sensitive but also quantitative detection of molecular biomarkers and live cells. In addition, there is an urgent need to design carbon-based "smart" SERS platforms as flexible and wearable SERS electronics that can disassociate into small pieces for rapid renal clearance. One of the ideal ways to achieve the goal of in vivo vital sign monitoring is noninvasive monitoring of human sweat secretion or blood detection. Due to the low target concentration, it is still a great challenge to analyze substances in human secretions and to develop ultrasensitive detection devices. Lost-cost and miniaturized devices will facilitate the adoption of SERS for medical applications or even for point-of-care-testing clinical human applications. Microfluidic chips are a new platform for controlling fluids on the micron or nanometer scale. Miniaturization, low reagent consumption, high efficiency, and easy integration with other devices are the main advantages of microfluidic chips. The combination of microfluidic chips and SERS technologies gives SERS microfluidic chips many competitive advantages, such as fast detection speed, high sensitivity, good selectivity, small sample volume, low cross-contamination, and simple 


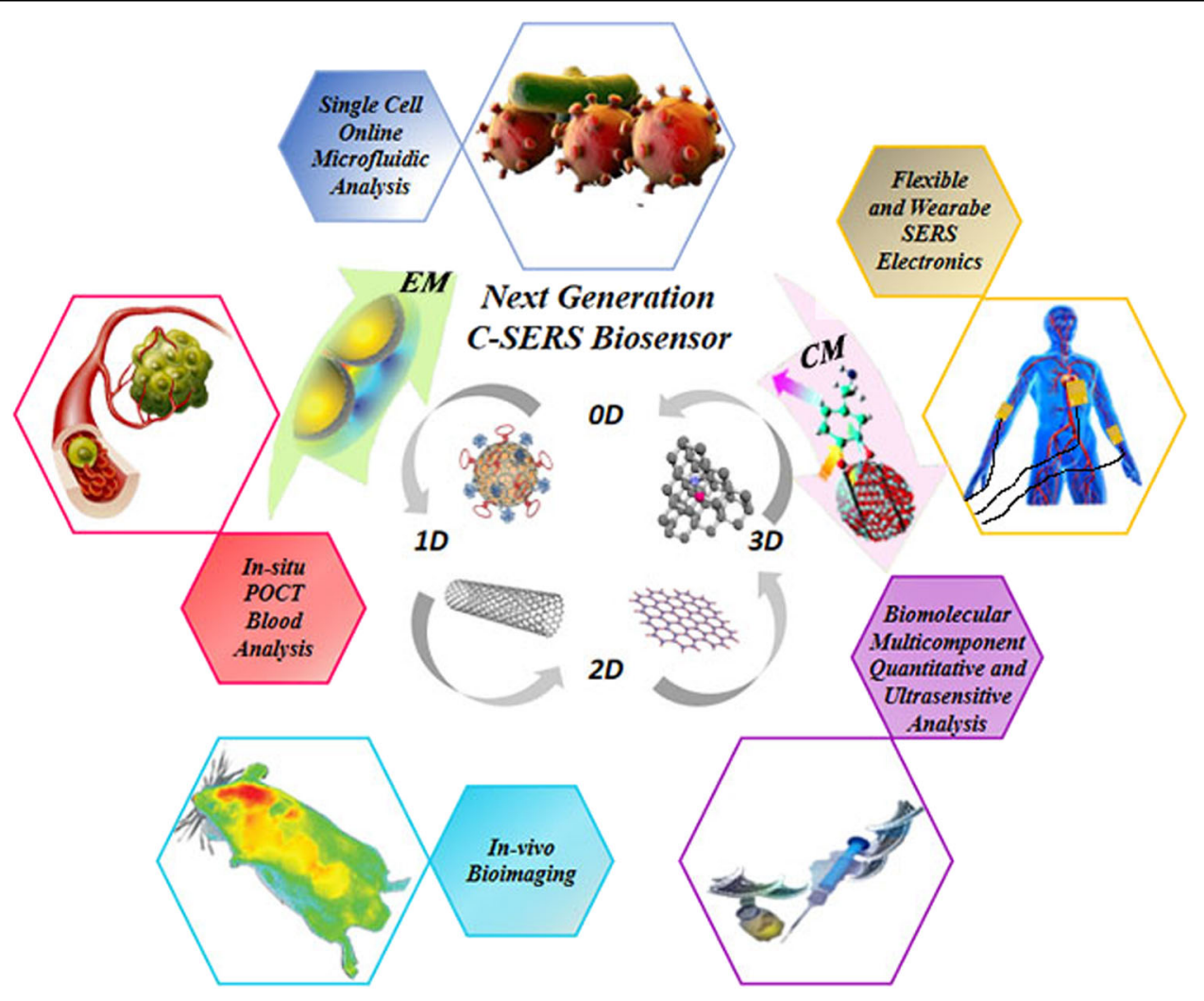

Fig. 15 Perspective on carbon-based SERS biosensors for potential future sensing applications.

operation. The integrated cross-cutting research on and development of these two technologies has attracted much attention. However, this combination technology also faces some challenges, such as how to ensure the uniformity of the SERS substrates to achieve accurate quantification, and the processing cost of the SERS substrate is still high. With the development of microfabrication technology, microfluidic chip technology, and droplet microfluidic technology, carbon-based SERS microfluidic chips will continue to improve and be rapidly developed toward portability, integration, automation, and industrialization. We believe that the function of carbon-based nanomaterials, intentionally or not, will bring SERS to a new stage in the future.

\section{Acknowledgements}

This work was supported by the National Key Research and Development Program of China (2017YFA0206901, 2017YFA0206900), the National Natural Science Foundation of China $(21705027,61903235)$, the Australia National Health and Medical Research Council (NHMRC, APP1163786), the Australian Research Council (ARC, DP190101008), the Research Starting funds from both Suzhou University of Science and Technology and Southwest University in China, and the Recruitment Program of Global Experts of China and the Thousand Talent Plan of Shanghai.

\section{Author details}

'National Supercomputer Research Center of Advanced Materials, Advanced Materials Institute, Qilu University of Technology (Shandong Academy of
Sciences), 250014 Jinan, People's Republic of China. ${ }^{2}$ Department of Chemistry, Laboratory of Advanced Materials, Shanghai Key Laboratory of Molecular Catalysis and Innovative Materials, Fudan University, 200433 Shanghai, People's Republic of China. ${ }^{3}$ Key Laboratory of Bio-Inspired Smart Interfacial Science and Technology of Ministry of Education, School of Chemistry, Beihang University, 100191 Beijing, People's Republic of China. ${ }^{4}$ School of Chemical Engineering and Graduate School of Biomedical Engineering, The University of New South Wales, Sydney, NSW 2052, Australia

\section{Conflict of interest}

The authors declare that they have no conflict of interest.

\section{Publisher's note}

Springer Nature remains neutral with regard to jurisdictional claims in published maps and institutional affiliations.

Received: 30 October 2019 Revised: 28 April 2020 Accepted: 26 July 2020. Published online: 22 January 2021

\section{References}

1. Hou, C., Zhao, D. Y., Wang, Y., Zhang, S. F. \& Li, S. Y. Preparation of magnetic Fe3O4/PPy@ZIF-8 nanocomposite for glucose oxidase immobilization and used as glucose electrochemical biosensor. J. Electroanal. Chem. 822, 50-56 (2018).

2. Palecek, E. et al. Electrochemistry of nonconjugated proteins and glycoproteins. Toward sensors for biomedicine and glycomics. Chem. Rev. 115 2045-2108 (2015).

3. Baluta, S., Lesiak, A. \& Cabaj, J. Graphene quantum dots-based electrochemical biosensor for catecholamine neurotransmitters detection. Electroanalysis 30, 1773-1782 (2018). 
4. You, M., Yang, S., Tang, W. X., Zhang, F. \& He, P. G. Molecularly imprinted polymers-based electrochemical DNA biosensor for the determination of BRCA-1 amplified by SiO2@Ag. Biosens. Bioelectron. 112, 72-78 (2018).

5. Strande, N. T. et al. Analytical and clinical validation of variants identified by exome sequencing through secondary review and sanger confirmation in a CLIA-certified molecular laboratory. J. Mol. Diagn. 19, 952-952 (2017).

6. Egan, J. B. et al. Indices of actionability and clinical utility in a CLIA-enabled study of whole genome/exome/RNA sequencing in 33 cancer patients: actionable vs. utility. Cancer Res. 74, 4694 (2014).

7. Yang, R. et al. Development of novel portable and reusable fiber optical chemiluminescent biosensor and its application for sensitive detection of microcystin-LR. Biosens. Bioelectron. 121, 27-33 (2018).

8. Wang, Y. H., Zhang, L. N., Kong, Q. K., Ge, S. G. \& Yu, J. H. Time-resolution addressable photoelectrochemical strategy based on hollow-channel paper analytical devices. Biosens. Bioelectron. 120, 64-70 (2018).

9. Niu, L. Q. et al. Sensitive fluorescent detection of methyltransferase based on thermosensitive poly(N-isopropylacrylamide). Talanta 189, 579-584 (2018).

10. Kim, S. W., Cho, I. H., Lim, G. S., Park, G. N. \& Paek, S. H. Biochemicalimmunological hybrid biosensor based on two-dimensional chromatography for on-site sepsis diagnosis. Biosens. Bioelectron. 98, 7-14 (2017).

11. Beitlich, T., Kühnel, K., Schulzebriese, C., Shoeman, R. L. \& Schlichting, I. Cryoradiolytic reduction of crystalline heme proteins: analysis by UV-Vis spectroscopy and X-ray crystallography. J. Synchrotron Radiat. 14, 11-23 (2007).

12. Kucerova, P., Komenska, P., Tomkova, H., Skopalova, J. \& Bartak, P. Determination of lactose in milk products: a comparison of three-enzyme amperometric biosensor and gas chromatography/tandem mass spectrometry Monatsh. Chem. 148, 517-524 (2017).

13. Wang, Y. \& Ni, Y. Combination of UV-vis spectroscopy and chemometrics to understand protein-nanomaterial conjugate: a case study on human serum albumin and gold nanoparticles. Talanta 119, 320 (2014).

14. Hun, Y. S., Li, H. W. \& Li, J. B. A novel electrochemical biosensor for HIV-related DNA detection based on toehold strand displacement reaction and cruciform DNA crystal. J. Electroanal. Chem. 822, 66-72 (2018).

15. Jin, Y. C. et al. Electrochemical-signal-amplification strategy for an electrochemiluminescence immunoassay with g-C3N4 as tags. Anal. Chem. 90, 12930-12936 (2018).

16. Zang, R. H., He, Y., Yuan, R. \& Chai, Y. Q. An ultrasensitive electrochemiluminescence immunosensor based on zeolitic imidazolate frameworks encapsulating spherical graphite crystals. J. Electroanal. Chem. $\mathbf{7 8 1}$ 284-288 (2016).

17. Tian, C. F., Deng, Y. H., Zhao, D. Y. \& Fang, J. X. Plasma silver supercrystals with ultrasmall nanogaps for ultrasensitive SERS-based molecule detection. Adv. Opt. Mater. 3, 404-411 (2015).

18. Schlucker, S. Surface-enhanced Raman spectroscopy: concepts and chemical applications. Angew. Chem. Int. Ed. 53, 4756-4795 (2014).

19. Koh, C. S. L. et al. SERS- and electrochemically active 3D plasma liquid marbles for molecular-level spectroelectrochemical investigation of microliter reactions. Angew. Chem. Int. Ed. 56, 8813-8817 (2017).

20. Sprague-Klein, E. A. et al. Observation of single molecule plasmon-driven electron transfer in isotopically edited 4,4'-bipyridine gold nanosphere oligomers. J. Am. Chem. Soc. 139, 15212-15221 (2017).

21. Zrimsek, A. B. et al. Single-molecule chemistry with surface- and tipenhanced Raman spectroscopy. Chem. Rev. 117, 7583-7613 (2017).

22. Khalil, I., Julkapli, N. M., Yehye, W. A., Basirun, W. J. \& Bhargava, S. K. Graphenegold nanoparticles hybrid-synthesis, functionalization, and application in a electrochemical and surface-enhanced Raman scattering biosensor. Materials 9. 406 (2016).

23. Zheng, H., Zheng Y., Yan F., Chen M. \& Li, P. Next-Generation Ultrasonic Theranostic Agents for Molecular Imaging and Therapy: Design, Preparation, and Biomedical Application (Springer, Singapore, 2016).

24. Botta, R., Upender, G., Sathyavathi, R., Rao, D. N. \& Bansal, C. Silver nanoclusters films for single molecule detection using Surface Enhanced Raman Scattering (SERS). Mater. Chem. Phys. 137, 699-703 (2013).

25. Kneipp, K. et al. Detection and identification of a single DNA base molecule using surface-enhanced Raman scattering (SERS). Phys. Rev. E 57 R6281-R6284 (1998)

26. Kneipp, K. et al. Single molecule detection using surface-enhanced Raman scattering (SERS). Phys. Rev. Lett. 78, 1667-1670 (1997).
27. Balasubramanian, K. \& Burghard, M. Biosensors based on carbon nanotubes. Anal. Bioanal. Chem. 385, 452-468 (2006).

28. Stankovich, S. et al. Graphene-based composite materials. Nature 442 282-286 (2006)

29. Ambrosi, A., Chua, C. K., Bonanni, A. \& Pumera, M. Electrochemistry of graphene and related materials. Chem. Rev. 114, 7150-7188 (2014).

30. Fang, Y. \& Wang, E. Electrochemical biosensors on platforms of graphene. Chem. Commun. 49, 9526-9539 (2013).

31. Qu, L. L. et al. Highly reproducible Ag NPS/CNT-intercalated GO membranes for enrichment and SERS detection of antibiotics. ACS Appl. Mater. Inter. 8 28180-28186 (2016)

32. Zhang, J., Zhang, X. L., Lai, C. H., Zhou, H. J. \& Zhu, Y. Silver-decorated aligned CNT arrays as SERS substrates by high temperature annealing. Opt. Express 22, 21157-21166 (2014).

33. Li, Y., Dykes, J., Gilliam, T. \& Chopra, N. A new heterostructured SERS substrate: free-standing silicon nanowires decorated with graphene-encapsulated gold nanoparticles. Nanoscale 9, 5263-5272 (2017).

34. Gupta, V. K. et al. A novel glucose biosensor platform based on Ag@AuNPs modified graphene oxide nanocomposite and SERS application. J. Colloid Interface Sci. 406, 231 (2013).

35. Zhang, Y. et al. One-pot green synthesis of Ag nanoparticles-graphene nanocomposites and their applications in SERS, $\mathrm{H}_{2} \mathrm{O} 2$, and glucose sensing RSC Adv. 2, 538-545 (2011)

36. Wang, P. Graphene-Plasma Hybrid Platform for Label-Free SERS Biomedical Detection. Dissertations \& Theses-Gradworks (2015).

37. Smalley, H. K. R. E. \& Heath, J. C60: Buckminsterfullerene. Nature 318, 162-163 (1985).

38. Yang, Y. B., Yang, X. D., Yang, Y. J. \& Yuan, Q. Aptamer-functionalized carbon nanomaterials electrochemical sensors for detecting cancer relevant biomolecules. Carbon 129, 380-395 (2018).

39. Power, A. C., Gorey, B., Chandra, S. \& Chapman, J. Carbon nanomaterials and their application to electrochemical sensors: a review. Nanotechnol. Rev. 7 19-41 (2018).

40. Baker, S. N. \& Baker, S. A. Luminescent carbon nanodots: emergent nanolights. Angew. Chem. Int. Ed. 49, 6726-6744 (2010).

41. Wang, G. T. et al. Structure dependent properties of carbon nanomaterials enabled fiber sensors for in situ monitoring of composites. Compos. Struct. 195, 36-44 (2018)

42. Liu, Z., Tabakman, S., Welsher, K. \& Dai, H. Carbon nanotubes in biology and medicine: in vitro and in vivo detection, imaging and drug delivery. Nano Res. 2, 85-120 (2009).

43. Guo, Z., Wang, Z. Y., Wang, H. H., Huang, G. Q. \& Li, M. M. Electrochemical sensor for Isoniazid based on the glassy carbon electrode modified with reduced graphene oxide-Au nanomaterials. Mater. Sci. Eng. C 57, 197-204 (2015).

44. Adhikari, B. R., Govindhan, M. \& Chen, A. C. Carbon nanomaterials based electrochemical sensors/biosensors for the sensitive detection of pharmaceutical and biological compounds. Sensors 15, 22490-22508 (2015).

45. Tang, Y. Z. et al. The novel carbon nanomaterials electrochemical sensor for determination of trace aluminum in human body fluids with 8hydroxyquinoline. IEEE Sens. J. 13, 3270-3275 (2013).

46. Li, H., Kang, Z., Liu, Y. \& Lee, S. T. Carbon nanodots: synthesis, properties and applications. J. Mater. Chem. 22, 24230-24253 (2012).

47. Dolati, S. et al. Selection of specific aptamer against enrofloxacin and fabrication of graphene oxide based label-free fluorescent assay. Anal. Biochem. 549, 124-129 (2018).

48. Zhang, Y., Gonçalves, H., Da, S. J. \& Geddes, C. D. Metal-enhanced photoluminescence from carbon nanodots. Chem. Commun. 47, 5313 (2017)

49. Bhunia, S. K., Zeiri, L., Manna, J., Nandi, S. \& Jelinek, R. Carbon-dot/silvernanoparticle flexible SERS-active films. ACS Appl. Mater. Inter. 8, 25637-25643 (2016).

50. Wang, C., Li, Y., Xu, Q. J. \& Luo, L. Ag@aggregation-induced emission dye core/shell nanostructures with enhanced one- and two-photon fluorescence. Opt. Mater. 72, 710-716 (2017).

51. Li, D. et al. Fluorescent/SERS dual-sensing and imaging of intracellular Zn2. Anal. Chim. Acta 1038, 148-156 (2018).

52. Yue, S. et al. SERS-fluorescence dual-mode $\mathrm{pH}$-sensing method based on Janus microparticles. ACS Appl. Mater. Inter. 9, 39699-39707 (2017).

53. Huo, S. F., Zhang, Z. C. \& Ma, Q. L. Surface enhanced Raman scattering on $\mathrm{SiO} 2 / \mathrm{Ag}$ nanoparticles aggregate and preparation of nitrogen-doped carbon 
dots by pyrolysis of Co(2,2'-bipyridine)(2)(dicyanamide)(2). J. Exp. Nanosci. 11, 669-680 (2016).

54. Niu, X. J. et al. Upconversion fluorescence-SERS dual-mode tags for cellular and in vivo imaging. ACS Appl. Mater. Inter. 6, 5152-5160 (2014).

55. Jun, B. H. et al. Silica core-based surface-enhanced Raman scattering (SERS) tag: advances in multifunctional SERS nanoprobes for bioimaging and targeting of biomarkers. Bull. Korean Chem. Soc. 36, 963-978 (2015).

56. Jeong, S. et al. Fluorescence-Raman dual modal endoscopic system for multiplexed molecular diagnostics. Sci. Rep. 5, 9455 (2015).

57. Wang, Z. et al. SERS-fluorescence joint spectral encoding using organic-metal-QD hybrid nanoparticles with a huge encoding capacity for high-throughput biodetection: putting theory into practice. J. Am. Chem. Soc. 134, 2993-3000 (2012).

58. Zou, F. M. et al. Dual-mode SERS-fluorescence immunoassay using graphene quantum dot labeling on one-dimensional aligned magnetoplasma nanoparticles. ACS Appl. Mater. Inter. 7, 12168-12175 (2015).

59. Jia, Q. Y. et al. Recent advances and prospects of carbon dots in cancer nanotheranostics. Mater. Chem. Front. 4, 449-471 (2020).

60. Nwahara, N., Achadu, O. J. \& Nyokong, T. In-situ synthesis of gold nanoparticles on graphene quantum dots-phthalocyanine nanoplatforms: first description of the photophysical and surface enhanced Raman scattering behaviour. J. Photochem. Photobiol. A 359, 131-144 (2018).

61. WU, J. X., Wang, P. J., Wang, F. H. \& Fang, Y. Investigation of the microstructures of graphene quantum dots (GQDs) by surface-enhanced Raman spectroscopy. Nanomaterials 8, 864 (2018).

62. Wu, D. et al. A novel sensitive and stable surface enhanced Raman scattering substrate based on a MoS2 quantum dot/reduced graphene oxide hybrid system. J. Mater. Chem. C 6, 12547-12554 (2018).

63. Qiu, H. W. et al. Self-cleaning SERS membrane for reusable and ultrasensitive molecular detection via integrating graphitic-carbon-nitride nanosheets and $\mathrm{Ag}$ nanospheres into hierarchical graphene layers that covered with graphitic-carbon-nitride quantum-dots. Appl. Surf. Sci. 489, 1010-1018 (2019).

64. $\mathrm{Xu}, \mathrm{Z}$. W. et al. Ultrathin electronic synapse having high temporal/spatial uniformity and an $\mathrm{Al}_{2} \mathrm{O}_{3} /$ graphene quantum dots $/ \mathrm{Al}_{2} \mathrm{O}_{3}$ sandwich structure for neuromorphic computing. NPG Asia Mater 11, 18 (2019).

65. Tang, L. B., Ji, R. B., Li, X. M., Teng, K. S. \& Lau, S. P. Energy-level structure of nitrogen-doped graphene quantum dots. J. Mater. Chem. C 1, 4908-4915 (2013).

66. Zhou, H. J., Zou, F. M., Tran, V. T. \& Lee, J. Simultaneous enhancement of Raman scattering and fluorescence emission on graphene quantum dotspiky magnetoplasma supra-particle composite films. RSC Adv. $\mathbf{5}$ 81753-81758 (2015).

67. Rajender, G. \& Giri, P. K. Formation mechanism of graphene quantum dots and their edge state conversion probed by photoluminescence and Raman spectroscopy. J. Mater. Chem. C 4, 10852-10865 (2016).

68. Liu, J. F., Qin, L. X., Kang, S. Z., Li, G. D. \& Li, X. Q. Gold nanoparticles/glycine derivatives/graphene quantum dots composite with tunable fluorescence and surface enhanced Raman scattering signals for cellular imaging. Mater. Des. 123, 32-38 (2017).

69. Liu, D. H. et al. Raman enhancement on ultra-clean graphene quantum dots produced by quasi-equilibrium plasma-enhanced chemical vapor deposition. Nat. Commun. 9, 193 (2018).

70. Li, Y. et al. An electrochemical avenue to green-luminescent graphene quantum dots as potential electron-acceptors for photovoltaics. Adv. Mater. 23, 776 (2011)

71. Hou, Y. X. et al. Electrical and Raman properties of p-type and n-type modified graphene by inorganic quantum dot and organic molecule modification. Sci. China Phys. Mech. 54, 416-419 (2011).

72. Apalkov, V. \& Chakraborty, T. Electronic Raman signatures of valley polarization, shell filling in graphene quantum dots. Europhys. Lett. 95, 17008 (2011).

73. Cheng, H. H. et al. Graphene-quantum-dot assembled nanotubes: a new platform for efficient raman enhancement. ACS Nano 6, 2237-2244 (2012).

74. Kim, S. et al. Size-dependence of Raman scattering from graphene quantum dots: interplay between shape and thickness. Appl. Phys. Lett. 102, 053108 (2013).
75. Wang, J., Gao, X. L., Sun, H. J., Su, B. W. \& Gao, C. J. Monodispersed graphene quantum dots encapsulated Ag nanoparticles for surface-enhanced Raman scattering. Mater. Lett. 162, 142-145 (2016).

76. Liu, X. G. et al. 3D nano-arrays of silver nanoparticles and graphene quantum dots with excellent surface-enhanced Raman scattering. Mater. Sci. Technol. Lond. 34, 679-687 (2018).

77. Zhao, Z. L. et al. Activatable fluorescence/MRI bimodal platform for tumor cell imaging via $\mathrm{MnO} 2$ nanosheet-aptamer nanoprobe. J. Am. Chem. Soc. 136, 11220-11223 (2014).

78. lijima, S. Helical microtubules of graphitic carbon. Nature 354, 56-58 (1991).

79. Jishi, R., Inomata, D., Nakao, K., Dresselhaus, M. \& Dresselhaus, G. Electronic and lattice properties of carbon nanotubes. J. Phys. Soc. Jpn. 63, 2252-2260 (1994).

80. Vaccarini, L. et al. Purification procedure of carbon nanotubes. Synth. Met. 103, 2492-2493 (1999)

81. Qian, Y., Cheng, Y., Ouyang, Y. M., Yuan, W. E. \& Fan, C. Y. Multilayered spraying and gradient dotting of nanodiamond-polycaprolactone guidance channels for restoration of immune homeostasis. NPG Asia Mater 11, 36 (2019).

82. Lefrant, S. et al. Raman and SERS studies of carbon nanotubes. Curr. Appl. Phys. 374, 325-334 (2002).

83. Krivchenko, V. A. et al. Carbon nanowalls decorated with silicon for lithiumion batteries. Carbon 50, 1438-1442 (2012).

84. Kong, J. et al. Nanotube molecular wires as chemical sensors. Science 287, 622-625 (2000).

85. Liu, C. D. et al. Suspended 3D AgNPs/CNT nanohybrids for the SERS application. Appl. Surf. Sci. 487, 1077-1083 (2019).

86. Irle, S., Witek, H. A., Shinohara, H. \& Morokuma, K. COMP 105-prediction of Raman spectra in novel polyyne@SWNT peapods using dispersionaugmented density-functional-tight-binding. Abstr. Pap. Am. Chem. Soc 234, 105 (2007)

87. Kao, C. C. \& Young, R. J. A Raman spectroscopic investigation of heating effects and the deformation behaviour of epoxy/SWNT composites. Compos. Sci. Technol. 64, 2291-2295 (2004).

88. Liu, T. \& Kumar, S. Quantitative characterization of SWNT orientation by polarized Raman spectroscopy. Chem. Phys. Lett. 378, 257-262 (2003).

89. Stepanian, S. G., Karachevtsev, V. A., Glamazda, A. Y., Dettlaff-Weglikowska, U. \& Adamowicz, L. Combined Raman scattering and ab initio investigation of the interaction between pyrene and carbon SWNT. Mol. Phys. 101 2609-2614 (2003).

90. Rafailov, P. M., Thomsen, C., Monev, M., Dettlaff-Weglikowska, U. \& Roth, S. Electrochemical functionalization of SWNT bundles in acid and salt media as observed by Raman and X-ray photoelectron spectroscopy. Phys. Stat. Sol. B 245, 1967-1970 (2008).

91. Lucas, M. \& Young, R. J. Raman spectroscopic study of the effect of strain on the radial breathing modes of carbon nanotubes in epoxy/SWNT composites. Compos. Sci. Technol. 64, 2297-2302 (2004).

92. Tsvetkov, M. Y. et al. Ag on carbon nanowalls mesostructures for SERS. Proc. SPIE 9450, 94501V-94508V (2015).

93. Liu, H. G. et al. Manipulating the functionalization surface of grapheneencapsulated gold nanoparticles with single-walled carbon nanotubes for SERS sensing. Carbon 140, 306-313 (2018).

94. $\mathrm{Xu}, \mathrm{Y}$. R. et al. Plasma heating induced by Au nanoparticles for quasi-ballistic thermal transport in multi-walled carbon nanotubes. Nanoscale 11, 7572-7581 (2019).

95. Qin, X. J. et al. Nanoconjugates of Ag/Au/carbon nanotube for alkynemeditated ratiometric SERS imaging of hypoxia in hepatic ischemia. Anal. Chem. 91, 4529-4536 (2019).

96. Llobet, E. Gas sensors using carbon nanomaterials: a review. Sens. Actuators B 179, 32-45 (2013).

97. Ostrovskaya, L. Y. et al. Characterization of different carbon nanomaterials promising for biomedical and sensor applications by the wetting method. Powder Metall. Met. Ceram. 42, 1-8 (2003).

98. Ling, X. et al. Can graphene be used as a substrate for Raman enhancement? Nano Lett. 10, 553-561 (2010).

99. Gulzar, A. et al. Bioapplications of graphene constructed functional nanomaterials. Chem. Biol. Interact. 262, 69-89 (2017).

100. Ling, X. et al. Lighting up the Raman signal of molecules in the vicinity of graphene related materials. Acc. Chem. Res. 48, 1862-1870 (2015). 
101. Ling, X. \& Zhang, J. First-layer effect in graphene-enhanced Raman scattering. Small 6, 2020-2025 (2010).

102. Xu, H., Xie, L., Zhang, H. \& Zhang, J. Effect of graphene Fermi level on the Raman scattering intensity of molecules on graphene. ACS Nano. 5, 5338 (2011).

103. Huang, S. X. et al. Molecular selectivity of graphene-enhanced Raman scattering. Nano Lett. 15, 2892-2901 (2015).

104. Yang, H. et al. Comparison of surface-enhanced Raman scattering on graphene oxide, reduced graphene oxide and graphene surfaces. Carbon $\mathbf{6 2}$, 422-429 (2013).

105. Ling, X. et al. Raman enhancement effect on two-dimensional layered materials: graphene, h-BN and MoS2. Nano Lett. 14, 3033-3040 (2014).

106. Xie, L. M., Ling, X., Fang, Y., Zhang, J. \& Liu, Z. F. Graphene as a substrate to suppress fluorescence in resonance Raman spectroscopy. J. Am. Chem. Soc. 131, 9890-9891 (2009)

107. Liang, X. et al. Tuning plasma and chemical enhancement for SERS detection on graphene-based Au hybrids. Nanoscale 7, 20188-20196 (2015).

108. Liu, Z. M. et al. pH-dependent surface-enhanced Raman scattering of aromatic molecules on graphene oxide. J. Raman Spectrosc. 44, 75-80 (2013)

109. Wang, W. et al. Simple synthesis method of reduced graphene oxide/gold nanoparticle and its application in surface-enhanced Raman scattering. Chem. Phys. Lett. 582, 119-122 (2013).

110. Govindhan, M., Amiri, M. \& Chen, A. Au nanoparticle/graphene nanocomposite as a platform for the sensitive detection of $\mathrm{NADH}$ in human urine. Biosens. Bioelectron. 66, 474-480 (2015).

111. Khan, M. S., Vishakante, G. D. \& Siddaramaiah, H. Gold nanoparticles: a paradigm shift in biomedical applications. Adv. Colloid Interface Sci. 199-200 44-58 (2013).

112. Han, J., Liu, Y. \& Guo, R. Facile synthesis of highly stable gold nanoparticles and their unexpected excellent catalytic activity for Suzuki-Miyaura crosscoupling reaction in water. J. Am. Chem. Soc. 131, 2060-2061 (2009).

113. Rasheed, P. A. \& Lee, J. S. Recent advances in optical detection of dopamine using nanomaterials. Microchim. Acta 184, 1239-1266 (2017).

114. Wang, Q. Q., Zhang, X. P., Huang, L., Zhang, Z. Q. \& Dong, S. J. One-pot synthesis of $\mathrm{Fe} 3 \mathrm{O} 4$ nanoparticle loaded 3D porous graphene nanocomposites with enhanced nanozyme activity for glucose detection. ACS Appl. Mater. Inter. 9, 7465-7471 (2017)

115. Liu, S. H., Lu, F., Xing, R. M. \& Zhu, J. J. Structural effects of Fe3O4 nanocrystals on peroxidase-like activity. Chem. Eur. J. 17, 620-625 (2011).

116. Jones, S., Sinha, S. S., Pramanik, A. \& Ray, P. C. Three-dimensional (3D) plasma hot spots for label-free sensing and effective photothermal killing of multiple drug resistant superbugs. Nanoscale 8, 18301-18308 (2016).

117. Liang, X. et al. Three-dimensional MoS2-NS@Au-NPs hybrids as SERS sensor for quantitative and ultrasensitive detection of melamine in milk. J. Raman Spectrosc. 49, 245-255 (2018)

118. Zhang, L., Jiang, C. \& Zhang, Z. Graphene oxide embedded sandwich nanostructures for enhanced Raman readout and their applications in pesticide monitoring. Nanoscale 5, 3773-3779 (2013).

119. Leem, J., Wang, M. C., Kang, P. \& Nam, S. W. Mechanically self-assembled three-dimensional graphene-gold hybrid nanostructures for advanced nanoplasma sensors. Nano Lett. 15, 7684-7690 (2015).

120. Kim, T. H., Lee, K. B. \& Choi, J. W. 3D graphene oxide-encapsulated gold nanoparticles to detect neural stem cell differentiation. Biomaterials 34, 8660-8670 (2013).

121. Li, J. F. et al. Shell-isolated nanoparticle-enhanced Raman spectroscopy. Sci. Found. China 464, 392 (2010).

122. Li, J. F., Anema, J. R., Wandlowski, T. \& Tian, Z. Q. Dielectric shell isolated and graphene shell isolated nanoparticle enhanced Raman spectroscopies and their applications. Chem. Soc. Rev. 44, 8399 (2015).

123. Mccreery, R. L. \& Cooper, J. B. Raman spectroscopy for chemical analysis. Appl. Spectrosc. 55, 295 (2001).

124. Chen, P. et al. pH-sensitive nanocarrier based on gold/silver core-shell nanoparticles decorated multi-walled carbon manotubes for tracing drug release in living cells. Biosens. Bioelectron. 75, 446-451 (2016).

125. Lee, J. H. et al. Synthesis, optical properties, and multiplexed Raman bioimaging of surface roughness-controlled nanobridged nanogap particles. Small 12, 4726-4734 (2016).

126. Li, D. et al. Ag@C core-shell colloidal nanoparticles prepared by the hydrothermal route and the low temperature heating-stirring method and their application in surface enhanced Raman scattering. J. Phys. Chem. C 116 12283-12294 (2012).

127. Yang, D. H. et al. Preparation and characterization of an ultrathin carbon shell coating a silver core for shell-isolated nanoparticle-enhanced Raman spectroscopy. Chem. Commun. 47, 5873-5875 (2011).

128. Xu, W. G. Mao, N. N. \& Zhang, J. Graphene: a platform for surface-enhanced Raman spectroscopy. Small 9, 1206-1224 (2013).

129. Li, J. F., Zhang, Y. J., Ding, S. Y., Panneerselvam, R. \& Tian, Z. Q. Core-Shell Nanoparticle-Enhanced Raman Spectroscopy. Chem. Rev. 117, 5002-5069 (2017).

130. Liu, Y. M., Hu, Y. \& Zhang, J. Few-layer graphene-encapsulated metal nanoparticles for surface-enhanced Raman spectroscopy. J. Phys. Chem. C 118 8993-8998 (2014).

131. Duan, B. et al. Surface enhanced Raman scattering by graphene-nanosheetgapped plasma nanoparticle arrays for multiplexed DNA detection. Nanoscale 7, 12606-12613 (2015)

132. Bian, X. et al. Fabrication of Graphene-isolated-Au-nanocrystal nanostructures for multimodal cell imaging and photothermal-enhanced chemotherapy. Sci. Rep. 4, 6093-6101 (2014).

133. Shen, A. G. et al. Triplex Au-Ag-C core shell nanoparticles as a novel Raman label. Adv. Funct. Mater. 20, 969-975 (2010).

134. Shen, A. G. et al. Surface-enhanced Raman spectroscopy in living plant using triplex Au-Ag-C core-shell nanoparticles. J. Raman Spectrosc. 42, 879-884 (2011).

135. Yang, N., You, T. T., Gao, Y. K., Lu, S. C. \& Yin, P. G. One-step preparation method of flexible metafilms on the water-oil interface: self-assembly surface plasmon structures for surface-enhanced Raman scattering detection. Langmuir 35, 4626-4633 (2019).

136. Lu, S. C. et al. Rapid fabrication of three-dimensional flower-like gold microstructures on flexible substrate for SERS applications. Spectrochim. Acto A 212, 371-379 (2019).

137. Gao, Y. K., You, T. T., Yang, N., Zhang, C. M. \& Yin, P. G. Superhydrophobic 3D Forest-like Ag microball/nanodendrite hierarchical structure as SERS sensor for rapid droplets detection. Adv. Mater Interfaces 6, 1801966 (2019).

138. Liang, X. et al. Interfacial synthesis of a three-dimensional hierarchical MoS2NS@Ag-NP nanocomposite as a SERS nanosensor for ultrasensitive thiram detection. Nanoscale 9, 8879-8888 (2017).

139. Liang, X. et al. Controlled assembly of one-dimensional MoO3@Au hybrid nanostructures as SERS substrates for sensitive melamine detection. CrystEngComm 18, 7805-7813 (2016).

140. Musumeci, A. et al. SERS of semiconducting nanoparticles (TiO2 hybrid composites). J. Am. Chem. Soc. 131, 6040-+ (2009).

141. Cong, S. et al. Noble metal-comparable SERS enhancement from semiconducting metal oxides by making oxygen vacancies. Nat. Commun. $\mathbf{6}$ 7800-7806 (2015)

142. Lane, L. A., Qian, X. M. \& Nie, S. M. SERS nanoparticles in medicine: from labelfree detection to spectroscopic tagging. Chem. Rev. 115, 10489-10529 (2015).

143. Dieringer, J. A., Lettan, R. B., Scheidt, K. A. \& Van Duyne, R. P. A frequency domain existence proof of single-molecule surface-enhanced Raman spectroscopy. J. Am. Chem. Soc. 129, 16249-16256 (2007).

144. Lin, X. et al. Rapid and simple detection of sodium thiocyanate in milk using surface-enhanced Raman spectroscopy based on silver aggregates. J. Raman Spectrosc. 45, 162-167 (2014)

145. Lee, P. C. \& Meisel, D. Adsorption and surface-enhanced Raman of dyes on silver and gold sols. J. Phys. Chem. C. 86, 3391-3395 (1982).

146. Turkevich, J., Stevenson, P. C. \& Hillier, J. The formation of colloidal gold. J. Phys. Chem. 57, 670-673 (1953).

147. Gao, Y. et al. CTAB-triggered Ag aggregates for reproducible SERS analysis of urinary polycyclic aromatic hydrocarbon metabolites. Chem. Commun. $\mathbf{5 5}$ 2146-2149 (2019)

148. Guo, P. et al. Plasma core-shell nanoparticles for SERS detection of the pesticide thiram: size- and shape-dependent Raman enhancement. Nanoscale 7, 2862-2868 (2015)

149. Zou, Y. X. et al. Isotopic graphene-isolated-Au-nanocrystals with cellular Raman-silent signals for cancer cell pattern recognition. Chem. Sci. 9, 2842-2849 (2018).

150. Banholzer, M. J. et al. Silver-based nanodisk codes. ACS Nano. 4, 5446-5452 (2010). 
151. Haynes, C. L., McFarland, A. D., Smith, M. T., Hulteen, J. C. \& Van Duyne, R. P. Angle-resolved nanosphere lithography: manipulation of nanoparticle size, shape, and interparticle spacing. J. Phys. Chem. B 106, 1898-1902 (2002).

152. Lee, S. J., Morrill, A. R. \& Moskovits, M. Hot spots in silver nanowire bundles for surface-enhanced Raman spectroscopy. J. Am. Chem. Soc. 128, 2200-2201 (2006)

153. Chen, Y. et al. Ultrathin plasmene nanosheets as soft and surface-attachable SERS substrates with high signal uniformity. Adv. Opt. Mater. 3, 919-924 (2015)

154. Si, K. J., Guo, P., Shi, Q. \& Cheng, W. Self-assembled nanocube-based plasmene nanosheets as soft sERS substrates towards direct quantitative drug identification on surfaces. Anal. Chem. 87, 5263-5269 (2015).

155. Yang, N., You, T. T., Gao, Y. K., Zhang, C. M. \& Yin, P. G. Fabrication of a flexible gold nanorod polymer metafilm via a phase transfer method as a SERS substrate for detecting food contaminants. J. Agric. Food Chem. 66, 6889-6896 (2018).

156. Zhang, C. M. et al. Hydrophobic paper-based SERS platform for direct-droplet quantitative determination of melamine. Food Chem. 287, 363-368 (2019).

157. Ouyang, L., Hu, Y. W., Zhu, L. H., Cheng, G. J. \& Irudayaraj, J. A reusable laser wrapped graphene-Ag array based SERS sensor for trace detection of genomic DNA methylation. Biosens. Bioelectron. 92, 755-762 (2017).

158. Xu, W. G. et al. Graphene-veiled gold substrate for surface-enhanced Raman spectroscopy. Adv. Mater. 25, 928-933 (2013).

159. Alamri, M., Sakidja, R., Goul, R., Ghopry, S. \& Wu, J. Z. Plasma Au nanoparticles on 2D Mos2/graphene van der Waals heterostructures for high-sensitivity surface-enhanced Raman spectroscopy. ACS Appl. Nano Mater. 2, 1412-1420 (2019)

160. Muehlethaler, C. et al. Ultrahigh Raman enhancement on monolayer MoS2. ACS Photonics 3, 1164-1169 (2016).

161. Xin, W. et al. Novel strategy for one-pot synthesis of gold nanoplates on carbon nanotube sheet as an effective flexible SERS substrate. ACS Appl. Mater. Inter. 9, 6246-6254 (2017)

162. Yan, T. T. et al. Controllable SERS performance for the flexible paper-like films of reduced graphene oxide. Appl. Surf. Sci. 419, 373-381 (2017).

163. Lin, D., Qin, T., Wang, Y., Sun, X. \& Chen, L. Graphene oxide wrapped SERS tags: multifunctional platforms toward optical labeling, photothermal ablation of bacteria, and the monitoring of killing effect. ACS Appl. Mater. Interfaces 6, 1320-1329 (2014).

164. Prekodravac, J. R. et al. Monolayer graphene films through nickel catalyzed transformation of fullerol and graphene quantum dots: a Raman spectroscopy study. Phys Scr. T162, 014030 (2014).

165. Yin., P. G. A DFT study on graphene-based surface-enhanced Raman spectroscopy of benzenedithiol adsorbed on gold/graphene. J. Raman Spectrosc 50, 1510-1518 (2019)

166. Ding, S. Y. et al. Nanostructure-based plasmon-enhanced Raman spectroscopy for surface analysis of materials. Nat. Rev. Mater. 1, 16021- (2016).

167. Wei, Q. L., Ni, H., Jin, X. \& Yuan, J. Graphene oxide wrapped gold nanorods for enhanced photo-thermal stability. RSC Adv. 5, 54971-54977 (2015).

168. Xie, L. M., Ling, X., Fang, Y., Zhang, J. \& Liu, Z. F. Graphene as a substrate to suppress fluorescence in resonance Raman spectroscopy. J. Am. Chem. Soc 131, 9890-+ (2009)

169. Xie, W., Qiu, P. H. \& Mao, C. B. Bio-imaging, detection and analysis by using nanostructures as SERS substrates. J. Mater. Chem. 21, 5190-5202 (2011).

170. Liang, $X$. et al. Direct observation of enhanced plasmon-driven catalytic reaction activity of Au nanoparticles supported on reduced graphene oxides by SERS. Phys. Chem. Chem. Phys. 17, 10176-10181 (2015).

171. Wang, H., Yang, R., Yang, L. \& Tan, W. Nucleic acid conjugated nanomaterials for enhanced molecular recognition. ACS Nano. 3, 2451 (2009).

172. Hu, R. et al. Nucleic acid-functionalized nanomaterials for bioimaging applications. J. Mater. Chem. 21, 16323-16334 (2011).

173. Chen, L. et al. Simultaneous determination of human enterovirus 71 and coxsackievirus B3 by dual-color quantum dots and homogeneous immunoassay. Anal. Chem. 84, 3200-3207 (2012)

174. Byun, J. Y. et al. The use of an engineered single chain variable fragment in a localized surface plasmon resonance method for analysis of the C-reactive protein. Chem. Commun. 49, 9497-9499 (2013)

175. Zhu, X., Liu, Y., Li, P., Nie, Z. \& Li, J. Applications of graphene and its derivatives in intracellular biosensing and bioimaging. Analyst 141, 4541-4553 (2016).

176. Kim, Y. I. et al. Simultaneous detection of EGFR and VEGF in colorectal cancer using Fluorescence-Raman Endoscopy. Sci. Rep. 7, 1035 (2017).
177. Thakor, A. S. et al. The fate and toxicity of Raman-active silica-gold nanoparticles in mice. Sci. Transl. Med. 3, 79ra33 (2011).

178. Hoshino, A. et al. Use of fluorescent quantum dot bioconjugates for cellular imaging of immune cells, cell organelle labeling, and nanomedicine: surface modification regulates biological function, including cytotoxicity. J. Artif. Organs 10, 149-157 (2007)

179. Pan, X. et al. A graphene oxide-gold nanostar hybrid based-paper biosensor for label-free SERS detection of serum bilirubin for diagnosis of jaundice. Biosens. Bioelectron. 145, 111713 (2019).

180. Li, C. N., Fan, P. D., Liang, A. H. \& Jiang, Z. L. Using Ca-doped carbon dots as catalyst to amplify signal to determine ultratrace thrombin by free-label aptamer-SERS method. Mater. Sci. Eng. C 99, 1399-1406 (2019).

181. Song, Y. C., Xu, T. L., Xu, L. P. \& Zhang, X. J. Nanodendritic gold/graphenebased biosensor for tri-mode miRNA sensing. Chem. Commun. $\mathbf{5 5}$ 1742-1745 (2019).

182. Khalil, I. et al. Graphene oxide and gold nanoparticle based dual platform with short DNA probe for the PCR free DNA biosensing using surfaceenhanced Raman scattering. Biosens. Bioelectron. 131, 214-223 (2019).

183. Xu, S. et al. Graphene isolated Au nanoparticle arrays with high reproducibility for high-performance surface-enhanced Raman scattering. Sens. Actuators B 222, 1175-1183 (2016).

184. Shicai, X. et al. Highly ordered graphene-isolated silver nanodot arrays as SERS substrate for detection of urinary nucleosides. Laser Phys. 25, 115601 (2015).

185. Zheng, H., Ni, D., Yu, Z. \& Liang, P. Preparation of SERS-active substrates based on graphene oxide/silver nanocomposites for rapid zdetection of I-Theanine. Food Chem. 217, 511-516 (2017).

186. Wuytens, P. C. et al. Gold nanodome SERS platform for label-free detection of protease activity. Faraday Discuss. 205, 345-361 (2017).

187. Siddhanta, S., Wrobel, M. S. \& Barman, I. Integration of protein tethering in a rapid and label-free SERS screening platform for drugs of abuse. Chem. Commun. 52, 9016-9019 (2016).

188. Pham, X. H. et al. Glucose detection using 4-mercaptophenyl boronic acidincorporated silver nanoparticles-embedded silica-coated graphene oxide as a SERS substrate. Biochip J. 11, 46-56 (2017).

189. Li, J.j, An, H.-q, Zhu, J. \& Zhao, J.-W. Detecting glucose by using the Raman scattering of oxidized ascorbic acid: the effect of graphene oxide-gold nanorod hybrid. Sens. Actuators B 235, 663-669 (2016).

190. Guo, Y. et al. Fabrication of Ag-Cu2O/reduced graphene oxide nanocomposites as surface-enhanced Raman scattering substrates for in situ monitoring of peroxidase-like catalytic reaction and biosensing. ACS Appl. Mater. Inter. 9, 19074-19081 (2017).

191. Ondera, T. J. \& li, A. T. H. Gold nanopopcorn attached single-walled carbon nanotube hybrid for rapid detection and killing of bacteria. J. Mater. Chem. $B$ 2, 7534-7543 (2014)

192. Ko, Y. C., Fang, H. Y. \& Chen, D. H. Fabrication of Ag/ZnO/reduced graphene oxide nanocomposite for SERS detection and multiway killing of bacteria. J. Alloy. Compd. 695, 1145-1153 (2017).

193. Li, Y. et al. Fast and green synthesis of silver nanoparticles/reduced graphene oxide composite as efficient surface-enhanced Raman scattering substrate for bacteria detection. Monatsh. Chem. 148, 1155-1163 (2017).

194. Keren, S. et al. Noninvasive molecular imaging of small living subjects using Raman spectroscopy. Proc. Natl Acad. Sci. USA 105, 5844-5849 (2008)

195. Kircher, M. F. et al. A brain tumor molecular imaging strategy using a new triple-modality MRI-photoacoustic-raman nanoparticle. Nat. Med. 18, 829 (2012).

196. Zhang, $\mathrm{H}$. et al. Graphene oxide-BaGdF5 nanocomposites for multi-modal imaging and photothermal therapy. Biomaterials 42, 66-77 (2015).

197. Fei, X. X. et al. Synthesis of Au NP@MoS2 quantum dots Core@Shell nanocomposites for SERS bio-analysis and label-free bio-imaging. Materials 10,650 (2017).

198. Liu, Q. H. et al. Cell imaging by graphene oxide based on surface enhanced Raman scattering. Nanoscale 4, 7084-7089 (2012).

199. Liu, Z. A. et al. Multiplexed multicolor Raman imaging of live cells with isotopically modified single walled carbon nanotubes. J. Am. Chem. Soc. 130, 13540-+ (2008).

200. Zhao, Q. N., Song, W., Zhao, B. \& Yang, B. Spectroscopic studies of the optical properties of carbon dots: recent advances and future prospects. Mater Chem. Front. 4, 472-488 (2020). 
201. Woo, M. A. et al. Multiplex immunoassay using fluorescent-surface enhanced Raman spectroscopic dots for the detection of bronchioalveolar stem cells in murine lung. Anal. Chem. 81, 1008-1015 (2009)

202. Chen, Y. W., Liu, T. Y., Chen, P. J., Chang, P. H. \& Chen, S. Y. A high-sensitivity and low-power theranostic nanosystem for cell SERS imaging and selectively photothermal therapy using Anti-EGFR-conjugated reduced graphene oxide/mesoporous silica/AuNPs nanosheets. Small 12, 1458-1468 (2016).

203. Wang, X., Wang, C., Cheng, L., Lee, S. T. \& Liu, Z. Noble metal coated singlewalled carbon nanotubes for applications in surface enhanced Raman scattering imaging and photothermal therapy. J. Am. Chem. Soc. 134, 7414-7422 (2012).

204. Ortega-Guerrero, A., Espinosa-Duran, J. M. \& Velasco-Medina, J. TRPV1 channel as a target for cancer therapy using CNT-based drug delivery systems. Eur. Biophys. J. Biophys. 45, 423-433 (2016).

205. Avti, P. et al. Molecular imaging and targeted therapy with the antibody functionalized Gd@SWNT. Cancer Res. 69, 5028 (2009).

206. Kamath, B. A., Das, M., Kuznetsova, L., Longtin, J. \& Ojima, I. FunctionalizedSWNT as a versatile platform for tumor-targeted drug delivery and dual therapy. Abstr. Pap. Am. Chem. Soc. 244, 292 (2012)

207. Kumar, V., Kukkar, D., Hashemi, B., Kim, K. H. \& Deep, A. Advanced functional structure-based sensing and imaging strategies for cancer detection: possibilities, opportunities, challenges, and prospects. Adv. Funct. Mater. 29, 1807859 (2019).

208. Robinson, J. T. et al. In vivo fluorescence imaging in the second near-infrared window with long circulating carbon nanotubes capable of ultrahigh tumor uptake. J. Am. Chem. Soc. 134, 10664-10669 (2012)

209. Robinson, J. T. et al. Ultrasmall reduced graphene oxide with high nearinfrared absorbance for photothermal therapy. J. Am. Chem. Soc. 133, 6825-6831 (2011).

210. Peng, F. et al. Silicon nanomaterials platform for bioimaging, biosensing, and cancer therapy. Acc. Chem. Res. 47, 612-623 (2014).

211. Chung, $C$. et al. Biomedical applications of graphene and graphene oxide. Acc. Chem. Res. 46, 2211 (2013).

212. Qiao, X. Z. et al. Selective surface enhanced Raman scattering for quantitative detection of lung cancer biomarkers in superparticle@MOF structure. Adv. Mater. 30, 1702275 (2018)

213. Ngo, H. T. et al. Label-free DNA biosensor based on SERS molecular sentinel on nanowave chip. Anal. Chemy. 85, 6378-6383 (2013).

214. Kim, W. et al. A label-free cellulose SERS biosensor chip with improvement of nanoparticle-enhanced LSPR effects for early diagnosis of subarachnoid hemorrhage-induced complications. Biosens. Bioelectron. 111 59-65 (2018).

215. Ramya, A. N., Ambily, P. S., Sujitha, B. S., Arumugam, M. \& Maiti, K. K. Single cell lipid profiling of Scenedesmus quadricauda CASA-CC202 under nitrogen starved condition by surface enhanced Raman scattering (SERS) fingerprinting. Algal Res. 25, 200-206 (2017).

216. Hanif, S. et al. Nanopipette-based SERS aptasensor for subcellular localization of cancer biomarker in single cells. Anal. Chem. 89, 9911-9917 (2017).

217. Gong, T. X. et al. Highly sensitive SERS detection and quantification of sialic acid on single cell using photonic-crystal fiber with gold nanoparticles. Biosens. Bioelectron. 64, 227-233 (2015)

218. Chen, Y. L., Ding, L., Song, W. Y., Yang, M. \& Ju, H. X. Protein-specific Raman imaging of glycosylation on single cells with zone-controllable SERS effect. Chem. Sci. 7, 569-574 (2016).

219. Chen, J. et al. Fabrication of large-area, high-enhancement SERS substrates with tunable interparticle spacing and application in identifying microorganisms at the single cell level. J. Phys. Chem. C 116, 3320-3328 (2012).

220. Bhamidipati, M., Cho, H. Y., Lee, K. B. \& Fabris, L. SERS-based quantification of biomarker expression at the single cell level enabled by gold nanostars and truncated aptamers. Bioconjugate Chem. 29, 2970-2981 (2018).

221. Vitol, E. A., Orynbayeva, Z., Friedman, G. \& Gogotsi, Y. Nanoprobes for intracellular and single cell surface-enhanced Raman spectroscopy (SERS). J. Raman Spectrosc. 43, 817-827 (2012).

222. Toccafondi, $C$. et al. Thin nanoporous alumina-based SERS platform for single cell sensing. Appl. Surf. Sci. 351, 738-745 (2015).

223. Dina, N. E., Colniță, A., Leopold, N. \& Haisch, C. Rapid single-cell detection and identification of bacteria by using surface-enhanced Raman spectroscopy. Analyst 27, 1782-1789 (2017).

224. Nolan, J. P. et al. Single cell analysis using surface enhanced Raman scattering (SERS) tags. Methods 57, 272-279 (2012).
225. Hanif, S. et al. Organic cyanide decorated SERS active nanopipettes for quantitative detection of hemeproteins and Fe3+ in single cells. Anal. Chem. 89, 2522-2530 (2017).

226. Gregas, M. K., Yan, F., Scaffidi, J., Wang, H. N. \& Vo-Dinh, T. Characterization of nanoprobe uptake in single cells: spatial and temporal tracking via SERS labeling and modulation of surface charge. Nanomed. Nanotechnol. 7, 115-122 (2011).

227. Graham, D., Larmour, I. \& Argueta, E. Detection of specific biomarkers within single cells using SERS and nanosensing. Abstr. Pap. Am. Chem. Soc. 240, 156 (2010).

228. Syme, C. D., Sirimuthu, N. M. S., Faley, S. L. \& Cooper, J. M. SERS mapping of nanoparticle labels in single cells using a microfluidic chip. Chem. Commun. 46, 7921-7923 (2010).

229. Stetciura, I. Y. et al. Composite SERS-based satellites navigated by optical tweezers for single cell analysis. Analyst 140, 4981-4986 (2015).

230. Shi, M. L. et al. SERS assay of telomerase activity at single-cell level and colon cancer tissues via quadratic signal amplification. Biosens. Bioelectron. 77, 673-680 (2016).

231. Shachaf, C. M. et al. A novel method for detection of phosphorylation in single cells by surface enhanced Raman scattering (SERS) using composite organic-inorganic nanoparticles (COINs). PLos ONE 4, e5206 (2009).

232. Kang, B., Austin, L. A. \& El-Sayed, M. A. Real-time molecular imaging throughout the entire cell cycle by targeted plasma-enhanced Rayleigh/ Raman Spectroscopy. Nano Lett. 12, 5369-5375 (2012).

233. Liang, O. W. et al. Label-free distinction between p53+/+ and p53-/colon cancer cells using a graphene based SERS platform. Biosens. Bioelectron. 118, 108-114 (2018).

234. Reza, K. K. et al. Parallel profiling of cancer cells and proteins using a graphene oxide functionalized ac-EHD SERS immunoassay. Nanoscale 10, 406 (2018).

235. Lan, C. Q. et al. Self-assembled nanoporous graphene quantum dot-Mn3O4 nanocomposites for surface-enhanced Raman scattering based identification of cancer cells. RSC Adv. 7, 18658-18667 (2017).

236. Ju, J. et al. Sustained and cost effective silver substrate for surface enhanced Raman spectroscopy based biosensing. Sci. Rep. 7, 6917 (2017).

237. Zhang, G. et al. Contribution of oligomer/carbon dots hybrid semiconductor nanoribbon to surface-enhanced Raman scattering property. Appl. Surf. Sci. 364, 660-669 (2016).

238. Zhang, X. \& Du, X. Carbon nanodot-decorated Ag@SiO2 nanoparticles for fluorescence and surface-enhanced Raman scattering immunoassays. ACS Appl. Mater. Interfaces 8, 1033-1040 (2016).

239. Panpan, Z. et al. One-step synthesis of large-scale graphene film doped with gold nanoparticles at liquid-air interface for electrochemistry and Raman detection applications. Langmuir ACS J. Surf. Colloids 30, 8980-8989 (2014).

240. Wang, P. et al. Label-free SERS selective detection of dopamine and serotonin using graphene-Au nanopyramid heterostructure. Anal. Chem. 87, 10255 (2015).

241. Fan, Z., Kanchanapally, R. \& Ray, P. C. Hybrid graphene oxide based ultrasensitive SERS probe for label-free biosensing. J. Phys. Chem. Lett. 4, 3813-3818 (2013).

242. He, S. et al. Graphene-based high-efficiency surface-enhanced Raman scattering-active platform for sensitive and multiplex DNA detection. Anal. Chem. 84, 4622-4627 (2012).

243. Manikandan, M., Nasser, A. H., Talib, A. \& Wu, H. F. Facile synthesis of gold nanohexagons on graphene templates in Raman spectroscopy for biosensing cancer and cancer stem cells. Biosens. Bioelectron. 55, 180 (2014).

244. Liu, Z., Hu, C., Li, S., Zhang, W. \& Guo, Z. Rapid intracellular growth of gold nanostructures assisted by functionalized graphene oxide and its application for surface-enhanced raman spectroscopy. Anal. Chem. 84, 10338-10344 (2012).

245. Ali, A., Hwang, E. Y., Choo, J. \& Lim, D. W. PEGylated nanographene-mediated metallic nanoparticle clusters for surface enhanced raman scattering-based biosensing. Analyst 143, 2604-2615 (2018).

246. Chen, H. L. et al. Fabrication of graphene and AuNP core polyaniline shell nanocomposites as multifunctional theranostic platforms for SERS real-time monitoring and chemo-photothermal therapy. Theranostics 6, 1096-1104 (2016).

247. Li, Y. et al. Fast and green synthesis of silver nanoparticles/reduced graphene oxide composite as efficient surface-enhanced Raman scattering substrate for bacteria detection. Monatsh. Chem. 148, 1-9 (2017).

248. Guo, S. J. et al. Carbon nanotube/silica coaxial nanocable as a threedimensional support for loading diverse ultra-high-density meta 
nanostructures: facile preparation and use as enhanced materials for electrochemical devices and SERS. Chem. Mater. 21, 2247-2257 (2009).

249. $\mathrm{Ma}, \mathrm{X}$. et al. Graphene oxide wrapped gold nanoparticles for intracellular Raman imaging and drug delivery. J. Mater. Chem. B 1, 6495-6500 (2013).

250. Chen, P. et al. A wide range optical pH sensor for living cells using Au@Ag nanoparticles functionalized carbon nanotubes based on SERS signals. Anal. Bioanal. Chem. 406, 6337-6346 (2014).
251. Camden, J. P. et al. Probing the structure of single-molecule surfaceenhanced Raman scattering hot spots. J. Am. Chem. Soc. 130, 12616 (2008).

252. Zhao, Y. et al. Gap-tethered Au@AgAu Raman tags for the ratiometric detection of MC-LR. Anal. Chem. 91, 7162-7172 (2019).

253. Chowdhury, A. K. M. R. H., Tan, B. \& Venkatakrishnan, K. SERS-active 3D interconnected nanocarbon web toward nonplasma in vitro sensing of HeLa cells and fibroblasts. ACS Appl. Mater. Inter. 10, 35715-35733 (2018). 